Utah State University

DigitalCommons@USU

\title{
Backcountry Campsite Environmental Changes and Effective Monitoring Practices: A Case Study in Kenai Fjords National Park
}

\author{
Shannon T. Wesstrom \\ Utah State University, shannon.wesstrom@usu.edu \\ Christopher Monz \\ Utah State University, chris.monz@usu.edu
}

Follow this and additional works at: https://digitalcommons.usu.edu/envs_stures

Part of the Environmental Monitoring Commons

\section{Recommended Citation}

Wesstrom, S. T., and C. Monz. 2021. Backcountry campsite environmental changes and effective monitoring practices: A case study in Kenai Fjords National Park. Natural Resource Report NPS/KEFJ/ NRR-2021/2289. National Park Service, Fort Collins, Colorado. https://doi.org/10.36967/nrr-2287087

This Report is brought to you for free and open access by the Environment and Society Student Works at DigitalCommons@USU. It has been accepted for inclusion in Environment and Society Student Research by an authorized administrator of DigitalCommons@USU. For more information, please contact digitalcommons@usu.edu.

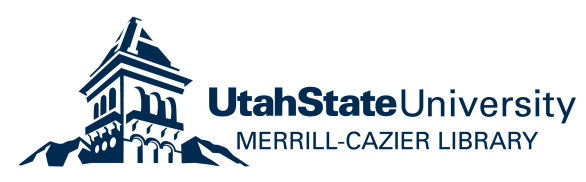


National Park Service

U.S. Department of the Interior

\section{Backcountry Campsite Environmental Changes and Effective Monitoring Practices}

A Case Study in Kenai Fjords National Park

Natural Resource Report NPS/KEFJ/NRR—2021/2289

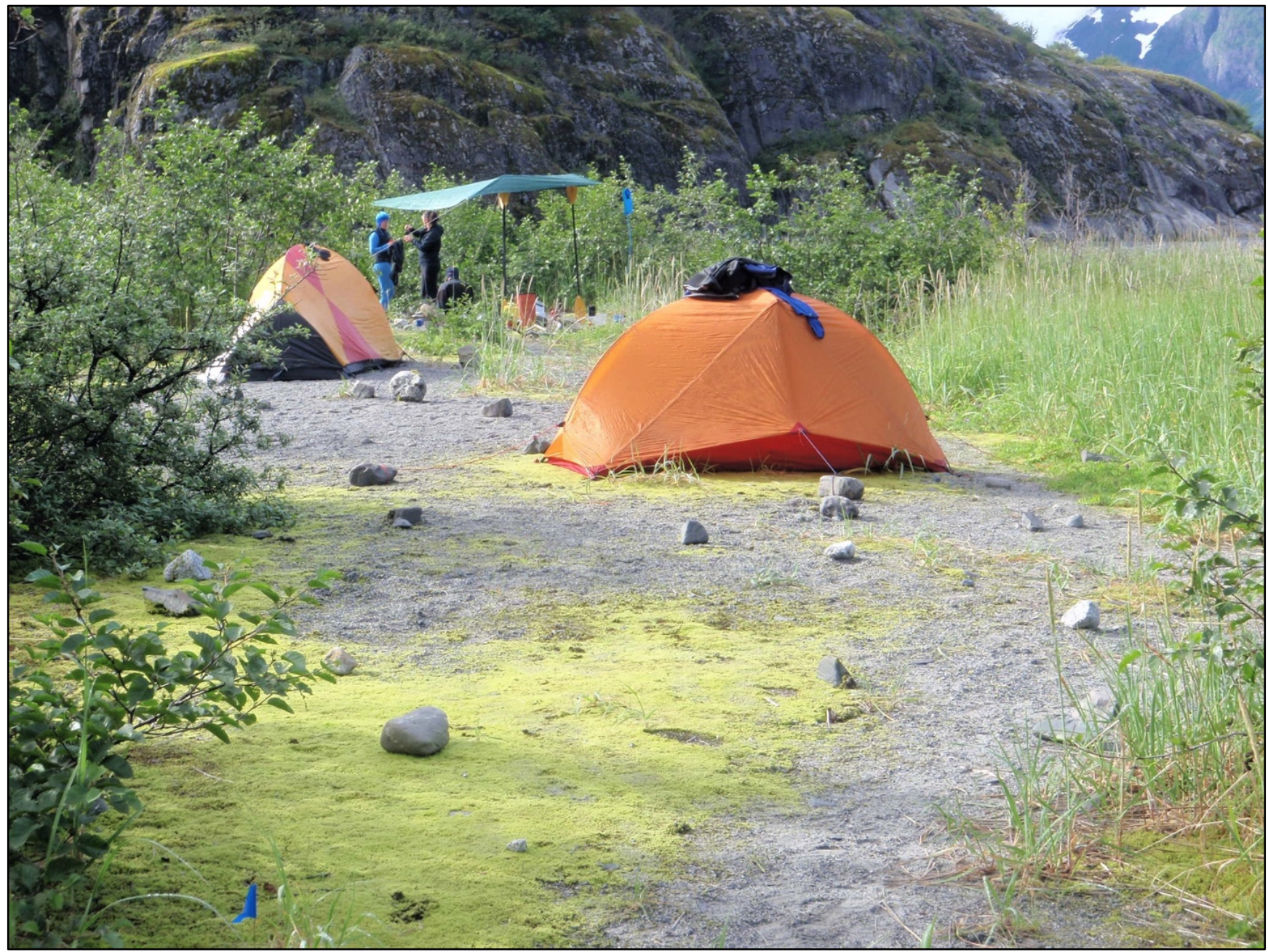


ON THE COVER

Campers in Kenai Fjords National Park at a backcountry campsite in Northwestern Fjord. NPS Photo 


\section{Backcountry Campsite Environmental Changes and Effective Monitoring Practices}

\section{A Case Study in Kenai Fjords National Park}

Natural Resource Report NPS/KEFJ/NRR—2021/2289

Shannon T. Wesstrom ${ }^{1}$, Christopher Monz ${ }^{2}$

${ }^{1}$ Masters Student

Utah State University

Department of Environment and Society

Logan, UT 84322

${ }^{2}$ Associate Professor

Utah State University

Department of Environment and Society

Logan, UT 84322

August 2021

U.S. Department of the Interior

National Park Service

Natural Resource Stewardship and Science

Fort Collins, Colorado 
The National Park Service, Natural Resource Stewardship and Science office in Fort Collins, Colorado, publishes a range of reports that address natural resource topics. These reports are of interest and applicability to a broad audience in the National Park Service and others in natural resource management, including scientists, conservation and environmental constituencies, and the public.

The Natural Resource Report Series is used to disseminate comprehensive information and analysis about natural resources and related topics concerning lands managed by the National Park Service. The series supports the advancement of science, informed decision-making, and the achievement of the National Park Service mission. The series also provides a forum for presenting more lengthy results that may not be accepted by publications with page limitations.

All manuscripts in the series receive the appropriate level of peer review to ensure that the information is scientifically credible, technically accurate, appropriately written for the intended audience, and designed and published in a professional manner.

This report received informal peer review by subject-matter experts who were not directly involved in analysis or reporting of the data. Data in this report were collected and analyzed using methods based on established, peer-reviewed protocols and were analyzed and interpreted within the guidelines of the protocols.

Views, statements, findings, conclusions, recommendations, and data in this report do not necessarily reflect views and policies of the National Park Service, U.S. Department of the Interior. Mention of trade names or commercial products does not constitute endorsement or recommendation for use by the U.S. Government.

This report is available in digital format from the Natural Resource Publications Management website. If you have difficulty accessing information in this publication, particularly if using assistive technology, please email irma@nps.gov.

Please cite this publication as:

Wesstrom, S. T., and C. Monz. 2021. Backcountry campsite environmental changes and effective monitoring practices: A case study in Kenai Fjords National Park. Natural Resource Report NPS/KEFJ/NRR—2021/2289. National Park Service, Fort Collins, Colorado.

https://doi.org/10.36967/nrr-2287087.

NPS 186/176999, August 2021 


\section{Contents}

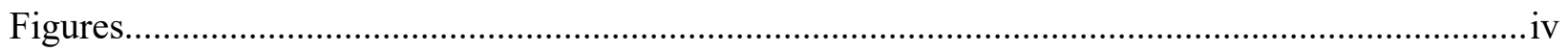

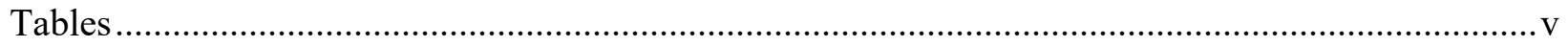

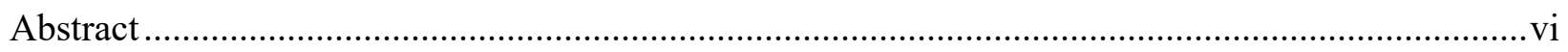

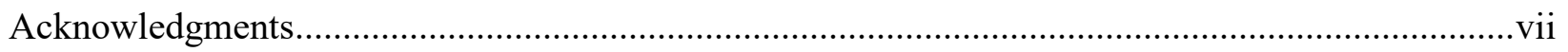

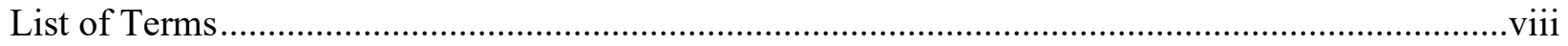

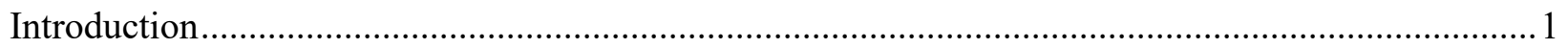

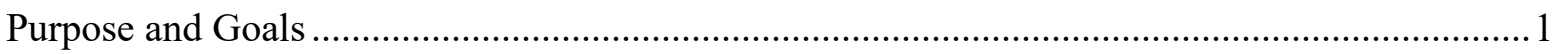

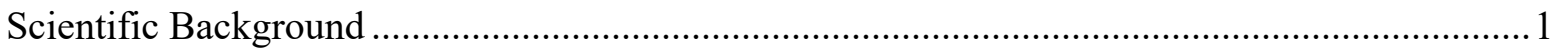

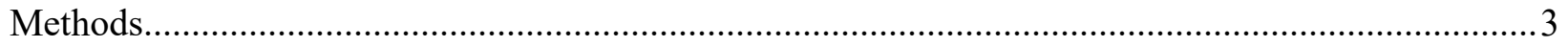

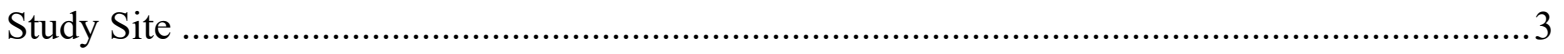

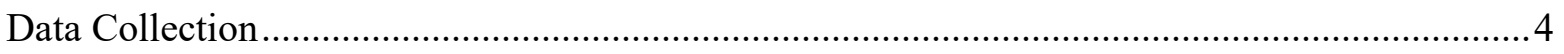

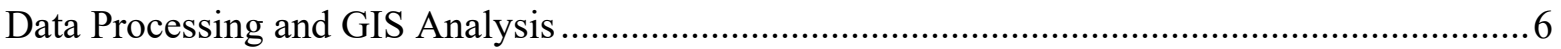

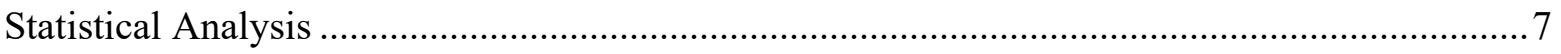

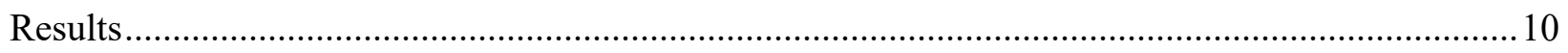

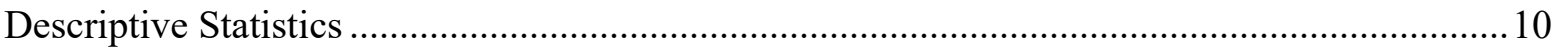

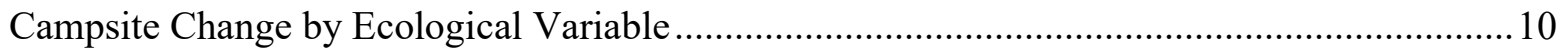

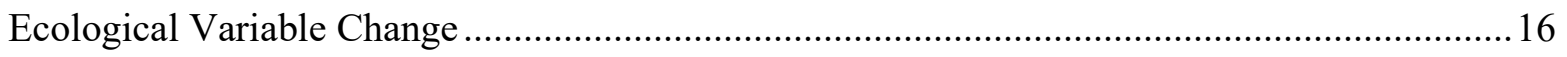

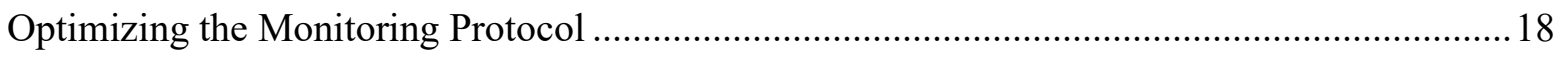

Redundant Ecological Variables ............................................................................ 18

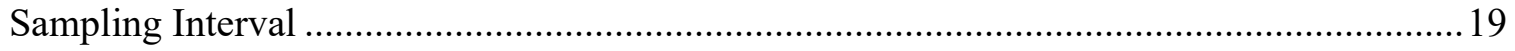

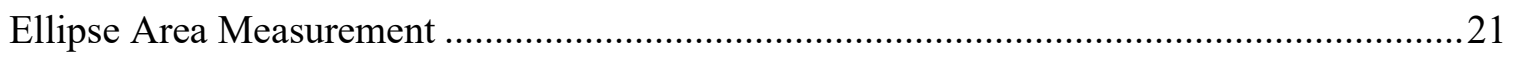

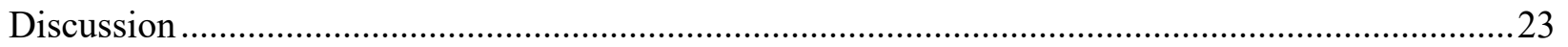

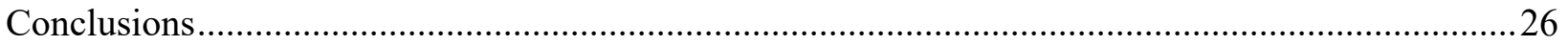

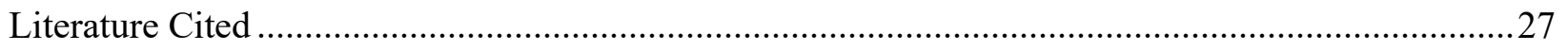

Appendix A. Annual Ecological Variable Changes by Campsite and Park Region ...........................30 


\section{Figures}

Figure 1. Beach locations of campsite study areas. ................................................................ 4

Figure 2. Drawn major and minor axes over theoretical campsite boundaries to create

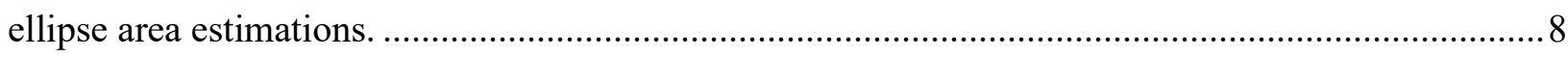

Figure 3. Yearly campsite area change by beach. Red campsite areas (NW Landing)

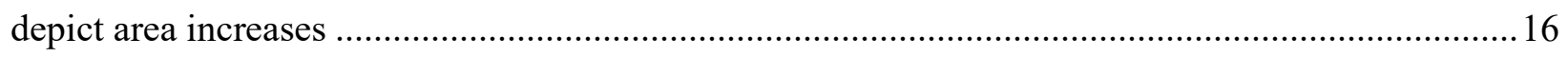

Figure 4. Scatterplot of campsites based on amount of change of variables in Factors 1 and 2 . 18

Figure 5. Simple linear regression model comparing radial area measurements to ellipse estimated area measurements 


\section{Tables}

Table 1. Impact assessment indicator variables, methods, and measurement scale..........................5

Table 2. Campsite condition class definitions (Monz et al. 2011, pg. 29) ...................................... 6

Table 3. Indicator variable summary for all sites in KEFJ ........................................................ 10

Table 4a. Indicator variable baseline (intercept) and annual change (slope) estimates by

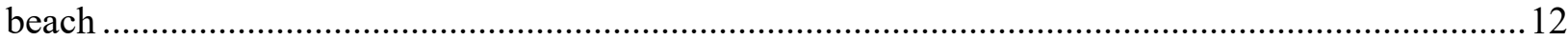

Table 4b. Indicator variable baseline (intercept) and annual change (slope) estimates by

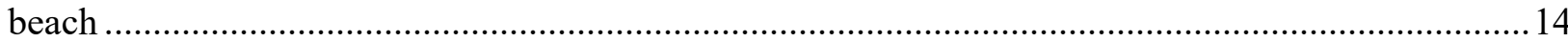

Table 5. Ranks of all indicator variables' change by beach........................................................ 15

Table 6. Factor analysis of indicator variables' change of campsites in KEFJ................................ 17

Table 7. Factor analysis of indicator variables' campsites in KEFJ. ............................................. 19

Table 8a. Continuous indicator variable change significance by sampling interval........................20

Table 8b. Categorical indicator variable change significance by sampling interval.......................21

Table 9. Descriptive statistics of different campsite area measurement methods. $\mathrm{N}=54 \ldots \ldots \ldots \ldots \ldots . .22$

Table 10. Paired samples correlations of radial versus estimated campsite area

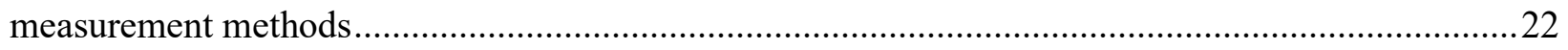

Table A1a. Indicator variable baseline (intercept) and annual change (slope) estimates by campsite

Table A1b. Indicator variable baseline (intercept) and annual change (slope) estimates by campsite

Table A2a. Indicator variable baseline (intercept) and annual change (slope) estimates by park region

Table A2b. Indicator variable baseline (intercept) and annual change (slope) estimates by park region 


\section{Abstract}

This report examines existing backcountry campsites' resource conditions over a five-year period in Kenai Fjords National Park (KEFJ), Alaska. Using campsite ecological monitoring techniques, 101 campsites were assessed for area size, vegetation cover loss, condition class assessments, as well as other indicator variable measurements. This research utilized parametric, non-parametric, robust linear regression, and principal component analysis statistical approaches to inform park managers of:

- Spatial and temporal patterns in changing campsite ecological variable conditions.

- Predicted annual variability of each ecological variable by campsite, beach, and bay.

- Opportunities for possible improvements in the efficiency of the current monitoring protocol by identifying:

○ An optimal sampling frequency.

- Key ecological variables to sample.

○ Areas of concern to focus sampling efforts.

Patterns in changing campsites were difficult to discern until examined by park region (i.e., bay). Tree damage, mineral soil exposure, and root exposure were indicator variables sensitive to change while campsite area displayed changes in some locations. Future monitoring protocols should replace the rapid and complete assessments with a streamlined comprehensive protocol that reduces the number of indicator variables to include: rapid campsite area measurements, tree damage, vegetation cover loss, tent rock counts, trail counts, condition class ratings, and ghost tree damage. Campsite assessments should be conducted at a three to five-year sampling interval and revised if large significant changes occur or there is a significant change in the level of visitor use. As parks and protected areas continue to see increases in visitation and overnight use, the potential for recreational impacts increases without the appropriate management strategies. Our conclusions provide evidence to determine suitable management approaches and can be applied to future monitoring protocols to ease the burden of time intensive and expensive sampling. 


\section{Acknowledgments}

We thank our field assistants including, K. Goonan, park officials, and park law enforcement, who helped to collect data in the field; Kenai Fjords National Park for the grant and overall opportunity to fulfill this research; C. Kriedeman, B. Pister, M. Hahr, L. Phillips, J. Cusick, and F. Klasner for their expertise, guidance, and support throughout the project. 


\section{List of Terms}

\section{Common acronyms:}

- PPA - Park and Protected Area

- KEFJ - Kenai Fjords National Park

- GPS - Global Positioning System

- GIS - Geographic Information System

\section{Ecological variables as defined by Monz et al. 2011 Coastal Campsite Monitoring} Protocol:

- Azimuth - The compass heading or "bearing" used in the radial transect method to determine campsite area.

- Campsite - Backcountry areas of disturbed vegetation, surface litter, or soils caused by use during overnight stays.

- Condition Class - Numerical categorization of campsite condition that refers to several ecological variables simultaneously: vegetation cover, mineral soil and root exposure, tree damage, trails, etc. inside the campsite perimeter.

- Fire Rings - Manmade rock formations to contain a camping fire.

- Ghost Tree - Dead standing tree stumps left behind from the 1964 Good Friday earthquake.

- High Tide Line - Marked by vegetation or sea debris at its highest point on the beach indicating the water level at high tide.

- Human Waste - Sightings of human feces and/or toilet paper.

- Mineral Soil - Soil devoid of the obvious organic layer and by general observation to consist only of mineral components (i.e., sand, silt, and clay).

- Substrate - A general term used to describe the soil-vegetation types where campsites and control plots are found.

○ Bedrock - Shelf bedrock.

○ Cobble - Fist sized rocks, larger than gravel, that do not move when you walk on them.

○ Sand - Sandy beach soils that do not form a surface crust in trampled areas.

- Sand/Cobble - Combination of sandy soils and cobble stones.

- Soil - Includes clay and loamy soils. Soil is a mixture of minerals, organic matter (dead and alive), water and air.

- Soil/Cobble - Combination of soil and cobble stones.

- Tent Rocks - Rocks used to stake out tents.

- Trails - Trampled vegetation or exposed soil leading to or from a campsite. 
- Trash - All recreation litter seen when standing in the center of the campsite, does not include flotsam.

- $\quad$ Tree/Ghost Tree Damage - Presence of scars, nails, cuts, fire burns as signified by a fire pit/site nearby all identified as human caused. Not windfall or bear damage related.

- Root Exposure - Tree root exposed due to trampling and erosion caused by human impacts.

- Vegetation Cover - Estimate percentage of the amount of live non-woody plants within the campsite and control plot perimeter. 


\section{Introduction}

\section{Purpose and Goals}

This assessment of the ecological condition of campsites from 2008 to 2012 in Kenai Fjords National Park combines several statistical applications to analyze changing conditions and attempts to optimize the monitoring protocol for the future.

Specific objectives for our research were:

- Determine the amount of change and annual variability by each campsite.

- Determine which ecological variables had the greatest amount of change during the sample period.

- Identify spatial patterns of change by campsite, beach, and park region.

- Enhance the current monitoring protocol by identifying key ecological indicators to sample and determine an optimized sampling frequency.

By analyzing a five-year dataset of coastal campsite assessments, we addressed the following questions:

- What is the magnitude of change of the sampled ecological variables over time in camping locations in Kenai Fjords National Park (KEFJ)?

- How can the monitoring protocol be optimized to reduce indicator redundancy, site observation intervals, and be more efficient in the field?

\section{Scientific Background}

Monitoring natural resource conditions is fundamental to park and protected area (PPA) management (Manning, 2011). Preserving the integrity of natural resources is the responsibility of PPA managers. With increasing demand for outdoor recreation (Machlis \& Tichnell, 2019), limiting the amount of ecological impact associated with increased visitation rates to recreation sites has grown more challenging. An effective and commonly used practice to evaluate recreational impacts, is to set up longitudinal monitoring programs. These programs assess changes in conditions and determine areas of concern based on the level of anthropogenic impacts. Study areas are commonly confined to popular visitor areas such as trails and campsites (Leung \& Marion, 2000).

Multiple studies have been conducted that suggest some generalizations of campsite effects on the environment (e.g. Twardock et al., 2010; Arredondo et al., 2018; Cole \& Hall, 1992). Cole and Hall (1992) discovered that even with increases in campsite size, vegetation cover remained consistent over an 11-year study period in Eagle Cap Wilderness, Oregon. Monz et al. (2010) summarized this phenomenon by explaining that on established sites, changes in areal extent or surface area were more obvious than the changes in the impact intensity. Increases in site numbers (which imply increases in surface area disturbances) over time may be more of a concern for managers than the degradation at the individual site level. This type of impact opens up a popular discussion for managers in terms of planning strategies. More confined "designated camping areas" limit the formation of new sites and allow for more desirable well-maintained sites, which work well in 
locations with high volumes of visitors (Brame \& Cole, 2011; Leung \& Marion, 1999). Conversely, Cole et al. (2008) found in a study of Grand Canyon National Park that, over 20 years, informal sites were created even under a functioning confinement campsite plan, resulting in an increase in total disturbed area. These results led managers to believe that a more dispersed campsite strategy plan works in areas where visitor campsite demand is low.

Dispersing or concentrating visitors to certain areas are not the only management strategies to reduce the amount of anthropogenic impacts. Hammitt et al. (2011) present type of recreational use, visitor behavior, timing of use, site hardening or shielding, and recreational site location as factors that can be manipulated by managers to reduce ecological impacts. When considering the composition of the recreational site location, vegetation morphology may be a better predictor of the resilience of a location than the amount of use (Cole, 1995a; 1995b; Shrader-Frechette et al., 1995). Numerous studies suggest that in terms of vegetation, the most resilient landscapes are those with rocky surfaces and grasses. Vegetation that is flexible, with rapid growth, and few stems tend to be the most resilient (Monz et al., 2013; Cole, 1995a; 1995b). Given that the ecological composition of many coastal camping locations in our study site are rocky, it is possible that there is little landcover disturbance caused by campers.

Kenai Fjords National Park (KEFJ) is in the unique position of offering experiences with receding tidewater glaciers, endangered wildlife, and backcountry adventures within a three hour drive of Anchorage, Alaska. Given the dispersal of overnight backcountry visitors in KEFJ, our study aims to investigate the potential ecological changes of campsites over a five-year sampling period. Determining the extent of ecological changes due to recreational influences in KEFJ helps managers improve backcountry experiences and may change how visitors are educated on Leave No Trace Principles to minimize damage. Additionally, while long term monitoring practices are vital to understanding the conditions of natural resources and how they might change due to certain management strategies, they are often expensive and time intensive. The original protocol for this project was designed to examine the ecological changes at campsites in KEFJ to a high degree of accuracy and precision. However, data collection often exceeded the time and resource expectations managers prepared for. Therefore, our study objectives were to inform managers of potential areas of concern in the backcountry landscape and how best to assess campsite conditions in the future.

The implications of our research test the applicability of Leung and Marion's (1999) multipleindicator monitoring system in a coastal Alaskan setting and are relevant to all campsite monitoring protocols. By investigating the rate and presence of ecological change and comparing the extent of change to each location we have a better understanding of appropriate monitoring intervals for park managers and the relationships between the variables at each campsite. Understanding visitor influence on the environment is integral for park managers to better establish guidelines to reduce the anthropogenic impacts on the environment. This analysis optimizes future monitoring programs by suggesting alternative data collection frequencies and establishing key indicators for observation to reduce cost and staffing needs. 


\section{Methods}

\section{Study Site}

Located in south central coastal Alaska, KEFJ provides a sanctuary for marine and terrestrial wildlife, a productive environment for colorful flora, and a dynamic geological landscape. Spanning $1,685 \mathrm{~km}^{2}, \mathrm{KEFJ}$ is less remote than other protected areas of Alaska. The Seward visitor center is less than a three hour drive from the city of Anchorage, making it more accessible to visitors because of the road system layout of the state. Unlike other protected areas of the state, visitors do not have to charter a plane or a boat to get to KEFJ once they are on the main Alaskan road system. Nearly 51\% of the park is covered in ice with 14 named glaciers within the park boundary (Nagorski et al., 2010). The dynamic landscape receives 203 to 381 centimeters of precipitation each year, establishing it as part of the temperate rainforest biome. Sitka spruce (Picea sitchensis) and Mountain hemlock (Tsuga mertensiana) are the dominant tree species in the region. While a majority of the tree species are coniferous, there are a few deciduous species including: black cottonwoods (Populus trichocarpa), Sitka alder (Alnus viridis ssp. sinuate), and several willow (Salix sp.) species (NPS, 2018; Boggs et al., 2008).

With harsh weather conditions from September to April, visitor use is typically confined to the summer months. Overnight visitors arrive at their campsites via kayak, motorized boats, or, on the rare occasion, sea planes. With the exception of the walk-in campground near Exit Glacier, there are no formal or designated campsites in the park. Most camping is confined to 15 beaches dispersed in the most popular three bays of KEFJ: Northwestern Fjord, Aialik Bay, and Resurrection Bay (Figure 1). These are the three most accessible bays due to their proximity to Seward and where most backcountry visitors camp and recreate in the park. Campsite substrate types on these beaches are classified as sand, soil, cobble, or some combination of the three. Soil/sandy beaches occur near a source of sediment deposition: rivers, eroding sea cliffs, and sand transported by wind or from the ocean shelf (Ritter, 1986). Above the high tide line, where visitors camp, the beach is dominated by grasses, forbs, and ferns. Species include: American dunegrass (Leymus mollis), beach pea (Lathyrus maritimus), lady fern (Athyrium filix), and alpine buckler fern (Dryopteris expansa). Further beyond the high tide line common graminoid vegetation species include: Hordeum bracteosum, Poa eminens, Festuca rubra, Deschampsia spp., and others. The graywacke (cobble) beaches are a result of erosion on rocky shorelines and cliffs and are often the remains of deglaciation. With exposure to wave action and storm swells common resilient species on these beaches include: American dune grass (Leymus mollis) and beach pea (Lathyrus maritimus), sea sandwort (Honckenya peploides), mountain hemlock (Tsuga mertensiana), Scottish licorice-root (Ligusticum scoticum), villous cinquefoil (Potentilla villosa), and lupine (Lupinus nootkatensis) (Boggs et al., 2008). 


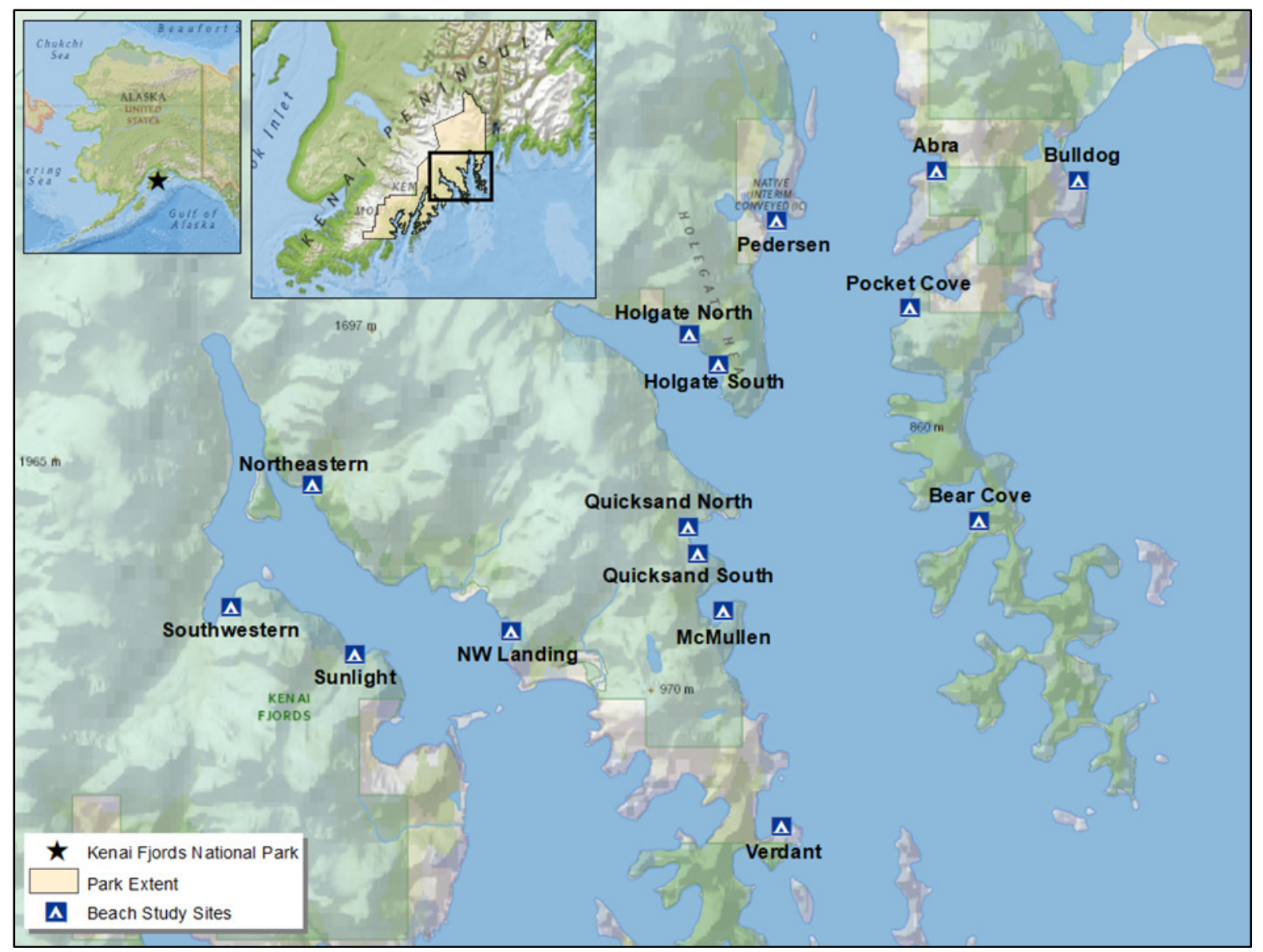

Figure 1. Beach locations of campsite study areas.

\section{Data Collection}

Monz et al. (2011) developed an Alaskan coast specific monitoring protocol following well established procedures created by Marion (1995), Leung and Marion (1999), Newsome et al. (2001), and previous work done by Monz and Twardock (2010). Data collection occurred from 2008 to 2012, with a sampling of 101 total observed campsites. To discern potential campsites, entire beaches feasible for camping were searched to find locations of flattened vegetation, surface layers, or soil disturbances that may indicate human influences. These search techniques occurred on the limited number of beaches able to accommodate camping. Combined with regular ranger patrols in most areas, this technique, provided a reasonable assurance that most camping locations were found and assessed.

Two types of campsite assessments were conducted as part of a long-term monitoring program for KEFJ: Rapid and Complete. Rapid assessments were designed to be done by one person and take approximately five minutes to complete per campsite. These quick assessments were intended to discover new sites not previously recorded and to check in on campsites the monitoring program was already aware of for large scale damages and severe ecological degradation. These assessments were planned to be performed in between regular monitoring field seasons to reduce the sampling burden 
(Monz et al., 2011). However, due to staff changes and the desire to acquire a more comprehensive data set, rapid assessments only occurred in 2008 with complete assessments taking place in 2009 through 2012. Complete assessments were designed for two to three technicians to complete in about fifteen to twenty minutes. Using a Trimble Global Positioning System (GPS) unit, several observations of the campsite were noted and recorded (Tables 1 and 2). Campsite area measurements were captured following Marion's (1995) radial transect protocol (Monz et al., 2011 pg. 39). The perimeter of the campsite was flagged and a metal center point marker with a unique identification number was buried in the center of the campsite. With a technician standing on the center point, the azimuth, or compass bearing from magnetic north, was recorded and the distance from the center point to each established boundary flag was measured. This process allowed for the determination of parameter points that could be georeferenced and plotted as polygons in real space after the data was collected. Photographs were also taken of the exact location to assist with the identification of the campsite in the following sampling years. All data were then uploaded, stored, and analyzed in GPS Pathfinder Office to be reviewed and evaluated later (Marion, 1995; Monz et al., 2011). While the intentions and requirements were straightforward for each assessment protocol, technicians in the field found the protocols to be more cumbersome than expected. Relocating the metal campsite center point pins proved to be the most difficult and time intensive. Combined with setting up the boundary flags and establishing the compass bearings, complete assessments took 30 minutes per campsite.

Table 1. Impact assessment indicator variables, methods, and measurement scale.

\begin{tabular}{|c|c|c|}
\hline Site Attribute & Method & Measurement Scale \\
\hline Campsite Area & Radial transect & Square Meters \\
\hline Distance from high tide & $\begin{array}{l}\text { Measurement of campsite } \\
\text { distance from high tide line } \\
\text { marked by vegetation }\end{array}$ & Meters \\
\hline Landing and campsite substrate type & Observation & $\begin{array}{l}\text { Sand, Sand/Cobble, Cobble, } \\
\text { Soil/Cobble, Soil, Bedrock }\end{array}$ \\
\hline Tree Canopy & Observation & Presence/Absence \\
\hline $\begin{array}{l}\text { Vegetation cover onsite/control and } \\
\text { mineral soil exposure onsite }\end{array}$ & Ocular estimation & $\begin{array}{l}\text { Six level scale: } 0-5 \%, 6-25 \%, 26- \\
50 \%, 51-75 \%, 76-95 \%, 96-100 \%\end{array}$ \\
\hline $\begin{array}{l}\text { Tree and ghost tree* damage and root } \\
\text { exposure onsite }\end{array}$ & Ocular estimation & $\begin{array}{l}\text { Four level scale: N/A, None/Slight, } \\
\text { Moderate, Severe }\end{array}$ \\
\hline $\begin{array}{l}\text { Tree and ghost tree* stumps, fire } \\
\text { rings, and trails }\end{array}$ & Counts & Total number present \\
\hline Tent Rocks & Ocular estimation & Four level scale: $0,1-5,6-15,16+$ \\
\hline Trash & Ocular estimation & $\begin{array}{l}\text { Three level scale: None to a handful, } \\
\text { more than a handful to a gallon, } \\
\text { greater than a gallon }\end{array}$ \\
\hline
\end{tabular}

\footnotetext{
* Ghost trees are the dead standing tree stumps left behind from the 1964 Good Friday earthquake. All three variables including root exposure, should only be assessed if trees are present.
} 
Table 1 (continued). Impact assessment indicator variables, methods, and measurement scale.

\begin{tabular}{lll}
\hline Site Attribute & Method & Measurement Scale \\
\hline Human Waste & Ocular estimation & Two level scale: None and Some \\
Condition Class & Ocular estimation & Six level classification scale \\
\hline
\end{tabular}

Table 2. Campsite condition class definitions (Monz et al. 2011, pg. 29).

\begin{tabular}{|c|c|}
\hline Class & Description \\
\hline \multirow[t]{4}{*}{ Class 0: } & $\begin{array}{l}\text { - Describes a previously established site that has re-grown and is not showing } \\
\text { current, observable disturbance. This class can only be used for re-measurement } \\
\text { of an established site }\end{array}$ \\
\hline & - Recreation site barely distinguishable \\
\hline & - None or minimal disturbance of vegetation and/or organic liter \\
\hline & - No observable vegetation loss in campsite as compared to off site \\
\hline \multirow[t]{3}{*}{ Class 1: } & - Recreation site barely distinguishable \\
\hline & - Slight loss of vegetation cover and/or minimal disturbance of organic liter \\
\hline & - $6-25 \%$ vegetation loss in campsite as compared to off site \\
\hline \multirow[t]{3}{*}{ Class 2: } & - Recreation site obvious \\
\hline & - Vegetation cover lost and/or organic litter pulverized in primary use areas \\
\hline & - $26-50 \%$ vegetation loss in campsite as compared to off site \\
\hline \multirow[t]{3}{*}{ Class 3: } & - Vegetation cover lost and/or organic litter pulverized on much of the site \\
\hline & - Some bare soil exposed in primary use areas \\
\hline & - $51-75 \%$ vegetation loss in campsite as compared to off site \\
\hline \multirow[t]{3}{*}{ Class 4: } & - Nearly complete or total loss of vegetation cover and organic liter \\
\hline & - Bare soil widespread \\
\hline & - $76-95 \%$ vegetation loss in campsite as compared to off site \\
\hline \multirow[t]{2}{*}{ Class 5: } & $\begin{array}{l}\text { - Soil erosion obvious, indicated by exposed tree roots and rocks and/or gullies } \\
\text { formed }\end{array}$ \\
\hline & - $96-100 \%$ vegetation loss in campsite as compared to off site \\
\hline
\end{tabular}

\section{Data Processing and GIS Analysis}

Polygons created from the radial transect measurements were imported into Esri's ArcMap 10.6.1 (2019, Environmental Systems Research Institute (ESRI), Redlands, CA, USA) and depicted campsite areas in meters squared. Center points, located in the center of each polygon, detailed the list of recorded variables (Monz et al., 2011). The polygons and center points were sorted by year of data collection and cleaned. Center points and polygons with notes indicating they were the new identification number for a center point marker that could not be found in the field were paired with the old center point marker number. For analysis purposes, campsites on beaches Verdant South and Verdant were combined due to their proximity to each other and to increase the sample size for a more accurate statistical analysis. Holgate Mid campsites were combined with Holgate South campsites for the same reason. While the majority of the indicator variables were recorded as 
numerical values or categories, vegetation cover loss needed to be calculated post hoc using vegetation cover estimates and the following equation (Monz \& Twardock, 2010):

$$
\text { Vegetation Cover Loss }=1-\frac{\% \text { cover in campsite }}{\% \text { cover in control plot }} \times 100 \%
$$

\section{Statistical Analysis}

Using a combination of parametric and non-parametric statistical analysis techniques, we determined which campsites, beaches, and regions had the most change during the study period, if there was redundancy in the observed indicator variables, and calculated more reasonable sampling intervals. A random coefficients model was used to estimate campsite ecological change by campsite, beach, and region over time. Campsites within a beach and beaches within a region were considered to be replicates. Each campsite, beach, and region was analyzed separately for each variable using a random coefficients model to estimate change per year as the linear slope coefficient. Random intercepts incorporated variance among campsites, however, there were too few repeated measures on campsites to estimate random slopes (Harrison et al., 2018). There were some limitations in definitively describing change by campsites due to smaller sample size, however, estimates for annual change were calculated. Since categorical variables used in this analysis were ordinal, they were recoded as integers. To determine which beach had the most ecological change, all estimated slopes for each indicator variable were ordered from most improved (negative integers) to most static (zero) or degrading (positive integers) and ranked. The data collection process was designed so each increase in category indicated progressive wear on the site. Positive values for change represented increased degradation and negative values for change indicated more recovery. Each variable ranking was added together to create one value to represent change rank amount for each beach. Each indictor held the same weight when being ranked to distinguish dynamic versus static beaches. This means, for example, campsite area had the same amount of pull in determining which beaches changed more as the amount of trash found on campsites.

Exploratory principal component analyses were conducted to visualize change occurring at campsites that were sampled more than once during the sampling period, using the estimated slopes data. Factor loadings were determined using varimax rotation and the results of the first two factors that describe the most variance were ordinated to illustrate patterns of change. A principal component analysis was used again using the cleaned raw data from each site, at each sampling year to determine if there was redundancy when measuring the independent indicator variables. This was an effort to determine if there were superfluous variables that could be excluded for a more efficient monitoring protocol (Monz \& Twardock, 2010; Leung \& Marion, 1999). Condition class rating assessments were not included in this analysis because of the covariance between these ratings and the other indicator variables. Condition class ratings were determined and provided as a generalization of all other indicator variables combined.

The data collection protocol specified intensive sampling measurements every other year at each campsite. The actual data collection sampling periods occurred more sporadically. Most sites were sampled in three-year increments (intensive sampling was completed three years apart). This proved to be beneficial for our study because it allowed us to determine a more appropriate intensive 
sampling interval. Paired student's T-tests were run on continuous variables, while Pearson's Chisquare tests were run for categorical data to determine which variables changed significantly over time (Twardock et al., 2010). Change significance of each indicator variable was conducted by interval sample group. Each campsite was assigned to one of four groups based on the time difference between its first sample and last. For example, if a site was first observed in 2008 and its last sample observation was in 2010, that site would be in the year 2 group. The sample number is often different for groups for each variable because there was missing data for some variables during sampling, but not all variables. By identifying when significant changes occurred for the variables, we determined a more efficient sampling interval.

Finally, to provide a more efficient method of measuring campsite area in the field, we compared the campsite area sizes that resulted from the radial transect method to estimated ellipses drawn around the polygons established by the radial transect method. To calculate the ellipse area, a major (a) and minor (b) axis of the campsite were drawn over the campsite polygon in ArcMap. The longest section from vertex to vertex was drawn as the major axis and the shortest section from vertex to vertex was drawn as the minor axis. Each axis aimed to cut the polygon in half trying to maintain equal parts on both sides (Figure 2). The estimated area was then calculated using the ellipse area equation:

$$
\text { Area }=\pi \frac{\mathrm{ab}}{2}
$$

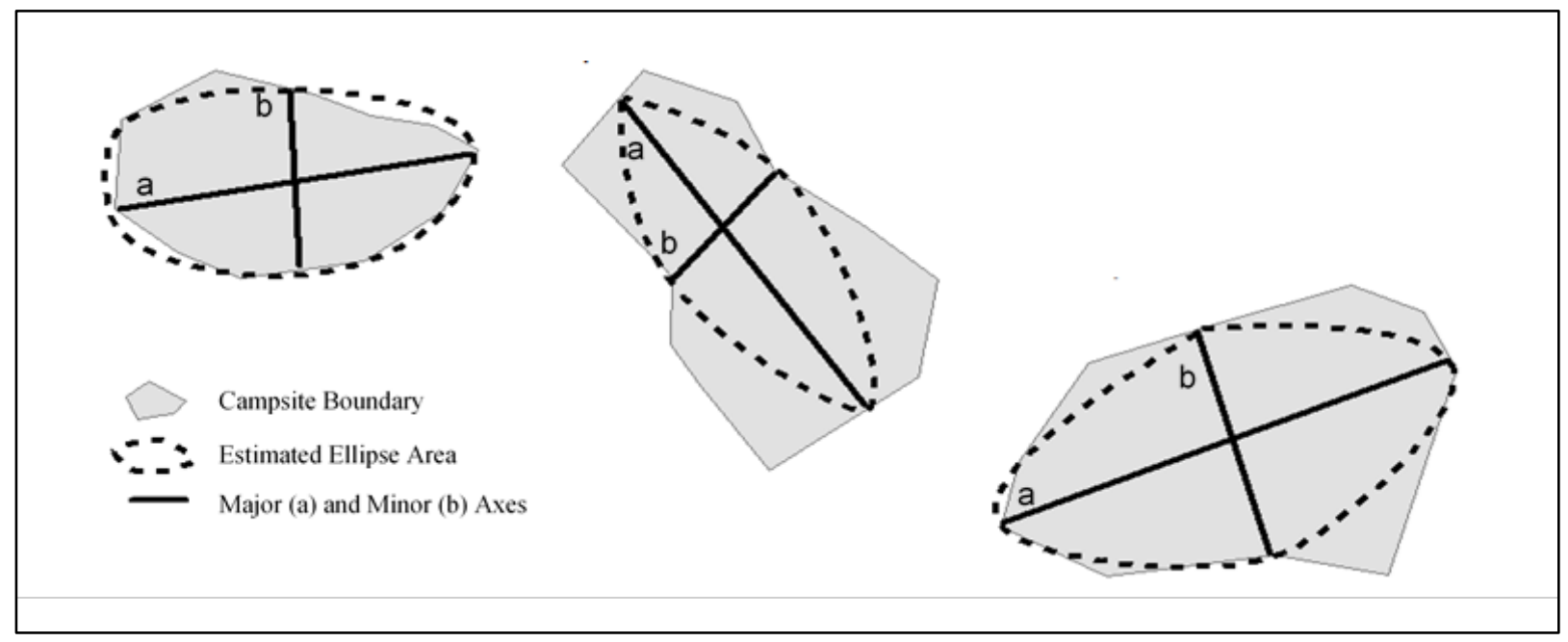

Figure 2. Drawn major and minor axes over theoretical campsite boundaries to create ellipse area estimations.

All 54 of the campsites measured in 2012 were used in the sample to determine if there was a significant difference in areas from the radial transect and ellipse methods. We calculated the means and standard deviations of both area measurements and compared the two with a paired T-Test to examine significant differences in the means. A simple linear regression analysis was also conducted in order to determine how well the ellipse areas could predict the true area determined by the radial 
transect. SPSS and R were used to summarize and conduct statistical analyses (v.25, IBM Corp., Armonk, NY, USA; v.1.1.456, R Foundation for Statistical Computing, Vienna, Austria). 


\section{Results}

\section{Descriptive Statistics}

Over the five-year study period, measurable impacts were found on 101 campsites. Based on the data collected from every campsite for each year sampled, the mean area for campsites in KEFJ was $31.46 \mathrm{~m}^{2}\left(\right.$ median $\left.=19.52 \mathrm{~m}^{2}\right)$. The mean condition class for all of the sites and their observations was two, indicating obvious recreational use. Vegetation loss and mineral soil exposure were both around $50 \%$. There was a mean of 8.47 tent rocks found at each site (Table 3). It is possible that tent rock numbers were inflated, because tent rocks were not counted individually but rather in categories.

Table 3. Indicator variable summary for all sites in KEFJ. Values are mean \pm SD for continuous variables and median \pm range for ordinal variables.

\begin{tabular}{llrr}
\hline Variable Type & Site Attribute & KEFJ Study Area & N \\
\hline Continuous & Area $\left(\mathrm{m}^{2}\right)$ & $31.46 \pm 41.85$ & 208 \\
& Condition Class & $2.14 \pm 1.06$ & 226 \\
& Vegetation Loss $(\%)$ & $55.66 \pm 39.22$ & 216 \\
& Mineral Soil Exposure (\%) & $58.56 \pm 37.15$ & 213 \\
& Tent Rocks & $8.47 \pm 5.98$ & 221 \\
& Trails & $2.19 \pm 1.63$ & 230 \\
& Fire Rings & $0.18 \pm 0.44$ & 230 \\
& Tree Stumps & $0.19 \pm 0.66$ & 230 \\
& Ghost Trees & $0.19 \pm 0.86$ & 230 \\
& Tree Damage & $0 \pm 2$ & 170 \\
Ordinal & Ghost Tree Damage & $1 \pm 2$ & 61 \\
& Root Exposure & $1 \pm 2$ & 173 \\
& Trash & $1 \pm 2$ & 229 \\
& Human Waste & $0 \pm 1$ & 229 \\
\hline
\end{tabular}

${ }^{\text {a }}$ Categorical Variables: $0=$ None/Slight, $1=$ Moderate, $2=$ Severe, NA $=$ Not Applicable.

${ }^{\mathrm{b}}$ Trash Variable: $0=$ None to a handful $1=$ Handful to a gallon, $2=$ Greater than a gallon .

${ }^{\mathrm{c}}$ Human Waste Variable: $0=$ None and $1=$ Some.

\section{Campsite Change by Ecological Variable}

A majority of variables were improving or remained static for each campsite. Trails and vegetation cover loss were the variables most likely to experience degradation. Number of tree stumps, fire rings, ghost tree damage, trash, and human waste predominately remained static. For these variables, if there was change, it was mostly improving. Campsites 93 (Pedersen) and 27 (Quicksand North) were the only two sampled sites that were degrading by four variables (area, vegetation loss, tent 
rocks, and mineral soil exposure), with the other variables remaining static. All other campsites either had a combination of recovering and static variables or a combination of recovering, static, and degrading variables (Tables $4 \mathrm{a}$ and $4 \mathrm{~b}$ ). Overall, 15 campsites recovered, and 14 new sites were found by the final year of sampling and no beaches experienced campsite proliferation. 
Table 4a. Indicator variable baseline (intercept) and annual change (slope) estimates by beach. Variable baseline of predicted 2008 value (Yearly Change). For all variables, a negative yearly change indicates improvement, 0 is no change, positive yearly change indicates degradation.

\begin{tabular}{|c|c|c|c|c|c|c|c|c|c|}
\hline Beach & Area $\left(\mathrm{m}^{2}\right)$ & $\begin{array}{r}\text { Condition } \\
\text { Class }\end{array}$ & $\begin{array}{r}\text { Vegetation } \\
\text { Loss (\%) }\end{array}$ & Tent Rocks & Trails & $\begin{array}{r}\text { Ghost Tree } \\
\text { Damage }\end{array}$ & Trash & $\begin{array}{l}\text { Human } \\
\text { Waste }\end{array}$ & $\begin{array}{r}\text { \# of } \\
\text { Campsites }\end{array}$ \\
\hline Abra & $\begin{array}{r}29.16 \\
(-2.41)\end{array}$ & $\begin{array}{r}3.48 \\
(-0.27)^{a}\end{array}$ & $\begin{array}{r}-64.06 \\
(7.78)\end{array}$ & $\begin{array}{r}16.80 \\
(-0.91)\end{array}$ & $\begin{array}{r}1.88 \\
(0.04)\end{array}$ & $\begin{array}{r}0.00 \\
(0.00)\end{array}$ & $\begin{array}{r}0.00 \\
(0.00)\end{array}$ & $\begin{array}{r}0.00 \\
(0.00)\end{array}$ & 7 \\
\hline Bear Cove & $\begin{array}{r}117.99 \\
(-19.27)^{a}\end{array}$ & $\begin{array}{r}3.97 \\
(-0.42)^{b}\end{array}$ & $\begin{array}{r}-40.29 \\
(1.60)\end{array}$ & $\begin{array}{r}8.05 \\
(0.10)\end{array}$ & $\begin{array}{r}3.82 \\
(-0.29)\end{array}$ & $\begin{array}{r}1.22 \\
(0.00)\end{array}$ & $\begin{array}{r}-0.03 \\
(0.25)^{b}\end{array}$ & $\begin{array}{r}0.00 \\
(0.00)\end{array}$ & 6 \\
\hline Bulldog & $\begin{array}{r}78.36 \\
(-12.78)^{a}\end{array}$ & $\begin{array}{r}3.57 \\
(-0.52)^{a}\end{array}$ & $\begin{array}{l}-44.10 \\
(-7.62)\end{array}$ & $\begin{array}{r}13.72 \\
(-1.22)\end{array}$ & $\begin{array}{r}1.52 \\
(0.03)\end{array}$ & $\begin{array}{r}0.105 \\
(-0.01)\end{array}$ & $\begin{array}{r}0.37 \\
(0.26)^{a}\end{array}$ & $\begin{array}{r}0.00 \\
(0.00)\end{array}$ & 5 \\
\hline Holgate North & $\begin{array}{r}53.59 \\
(-8.71)\end{array}$ & $\begin{array}{r}4.09 \\
(-0.78)^{b}\end{array}$ & $\begin{array}{c}-29.47 \\
(-7.33)^{a}\end{array}$ & $\begin{array}{r}19.09 \\
(-3.15)^{a}\end{array}$ & $\begin{array}{r}4.17 \\
(-0.83)^{b}\end{array}$ & $\begin{array}{r}0.18 \\
(-0.04)\end{array}$ & $\begin{array}{r}-0.10 \\
(0.26)^{b}\end{array}$ & $\begin{array}{r}0.00 \\
(0.00)\end{array}$ & 6 \\
\hline Holgate South & $\begin{array}{r}51.96 \\
(-9.90)^{a}\end{array}$ & $\begin{array}{r}5.14 \\
(-0.90)^{a}\end{array}$ & $\begin{array}{l}-13.26 \\
(-4.08)\end{array}$ & $\begin{array}{r}7.02 \\
(-0.75)\end{array}$ & $\begin{array}{r}1.89 \\
(-0.23)\end{array}$ & $\begin{array}{r}0.42 \\
(0.00)\end{array}$ & $\begin{array}{r}0.00 \\
(0.00)\end{array}$ & $\begin{array}{r}0.00 \\
(0.00)\end{array}$ & 3 \\
\hline McMullen & $\begin{array}{r}26.48 \\
(-2.99)\end{array}$ & $\begin{array}{r}1.54 \\
(0.04)\end{array}$ & $\begin{array}{r}-67.63 \\
(0.00)\end{array}$ & $\begin{array}{r}1.14 \\
(2.85)\end{array}$ & $\begin{array}{r}0.60 \\
(0.13)\end{array}$ & $\begin{array}{r}0.92 \\
(-0.13)\end{array}$ & $\begin{array}{r}-0.11 \\
(0.25)^{b}\end{array}$ & $\begin{array}{r}0.28 \\
(-0.06)\end{array}$ & 4 \\
\hline Northeastern & $\begin{array}{r}99.82 \\
(-10.35)^{a}\end{array}$ & $\begin{array}{r}2.92 \\
(-0.13)\end{array}$ & $\begin{array}{r}-78.13 \\
(6.25)\end{array}$ & $\begin{array}{r}12.47 \\
(-0.75)\end{array}$ & $\begin{array}{r}1.86 \\
(0.21)^{a}\end{array}$ & $\begin{array}{r}0.00 \\
(0.00)\end{array}$ & $\begin{array}{r}0.00 \\
(0.00)\end{array}$ & $\begin{array}{r}0.00 \\
(0.00)\end{array}$ & 11 \\
\hline NW Landing & $\begin{array}{l}14.89 \\
(2.22)\end{array}$ & $\begin{array}{r}0.25 \\
(0.75)\end{array}$ & $\begin{array}{l}-70.03 \\
(-1.77)\end{array}$ & $\begin{array}{r}6.75 \\
(-0.25)\end{array}$ & $\begin{array}{r}1.00 \\
(0.75)\end{array}$ & $\begin{array}{r}0.00 \\
(0.00)\end{array}$ & $\begin{array}{r}0.00 \\
(0.00)\end{array}$ & $\begin{array}{r}0.00 \\
(0.00)\end{array}$ & 4 \\
\hline Pedersen & $\begin{array}{r}63.74 \\
(-9.84)\end{array}$ & $\begin{array}{r}2.42 \\
(-0.04)\end{array}$ & $\begin{array}{r}-57.68 \\
(0.23)\end{array}$ & $\begin{array}{r}4.22 \\
(-0.23)\end{array}$ & $\begin{array}{r}2.79 \\
(0.24)\end{array}$ & $\begin{array}{r}0.64 \\
(-0.11)\end{array}$ & $\begin{array}{r}0.00 \\
(0.00)\end{array}$ & $\begin{array}{r}0.00 \\
(0.00)\end{array}$ & 13 \\
\hline Pocket Cove & $\begin{array}{r}62.30 \\
(-9.36)\end{array}$ & $\begin{array}{r}4.16 \\
(-0.58)\end{array}$ & $\begin{array}{r}-40.05 \\
(7.83)\end{array}$ & $\begin{array}{r}14.76 \\
(-0.88)\end{array}$ & $\begin{array}{l}-0.55 \\
(0.32)\end{array}$ & $\begin{array}{r}1.39 \\
(-0.17)\end{array}$ & $\begin{array}{r}0.00 \\
(0.00)\end{array}$ & $\begin{array}{r}0.00 \\
(0.00)\end{array}$ & 2 \\
\hline Quicksand North & $\begin{array}{r}30.82 \\
(-2.01)\end{array}$ & $\begin{array}{r}3.13 \\
(-0.13)\end{array}$ & $\begin{array}{r}-40.17 \\
(6.75)\end{array}$ & $\begin{array}{r}0.56 \\
(0.56)\end{array}$ & $\begin{array}{r}3.50 \\
(-0.25)\end{array}$ & $\begin{array}{r}0.63 \\
(-0.13)\end{array}$ & $\begin{array}{r}0.00 \\
(0.00)\end{array}$ & $\begin{array}{r}0.00 \\
(0.00)\end{array}$ & 4 \\
\hline
\end{tabular}

a P-value $<0.05$

${ }^{b}$ P-value $<0.001$ 
Table 4a (continued). Indicator variable baseline (intercept) and annual change (slope) estimates by beach. Variable baseline of predicted 2008 value (Yearly Change). For all variables, a negative yearly change indicates improvement, 0 is no change, positive yearly change indicates degradation.

\begin{tabular}{|c|c|c|c|c|c|c|c|c|c|}
\hline Beach & Area $\left(m^{2}\right)$ & $\begin{array}{r}\text { Condition } \\
\text { Class }\end{array}$ & $\begin{array}{r}\text { Vegetation } \\
\text { Loss (\%) }\end{array}$ & Tent Rocks & Trails & $\begin{array}{r}\text { Ghost Tree } \\
\text { Damage }\end{array}$ & Trash & $\begin{array}{r}\text { Human } \\
\text { Waste }\end{array}$ & $\begin{array}{r}\text { \# of } \\
\text { Campsites }\end{array}$ \\
\hline Quicksand South & $\begin{array}{r}45.98 \\
(-7.69)^{\mathrm{a}}\end{array}$ & $\begin{array}{r}4.65 \\
(-0.80)^{b}\end{array}$ & $\begin{array}{r}-45.99 \\
(5.07)\end{array}$ & $\begin{array}{r}14.05 \\
(-2.33)^{a}\end{array}$ & $\begin{array}{r}3.25 \\
(-0.37)\end{array}$ & $\begin{array}{r}1.79 \\
(-0.36)^{\mathrm{a}}\end{array}$ & $\begin{array}{r}0.00 \\
(0.00)\end{array}$ & $\begin{array}{r}0.00 \\
(0.00)\end{array}$ & 7 \\
\hline Southwestern & $\begin{array}{r}14.12 \\
(-0.76)\end{array}$ & $\begin{array}{r}2.02 \\
(0.00)\end{array}$ & $\begin{array}{r}-43.42 \\
(5.74)\end{array}$ & $\begin{array}{l}14.30 \\
(0.06)\end{array}$ & $\begin{array}{r}0.07 \\
(0.31)\end{array}$ & $\begin{array}{r}0.00 \\
(0.00)\end{array}$ & $\begin{array}{r}0.00 \\
(0.00)\end{array}$ & $\begin{array}{r}0.00 \\
(0.00)\end{array}$ & 6 \\
\hline Verdant & $\begin{array}{r}59.19 \\
(-9.19)^{a}\end{array}$ & $\begin{array}{r}3.77 \\
(-0.58)^{a}\end{array}$ & $\begin{array}{r}-18.48 \\
(1.65)\end{array}$ & $\begin{array}{r}17.08 \\
(-2.07)^{a}\end{array}$ & $\begin{array}{r}1.19 \\
(0.01)\end{array}$ & $\begin{array}{r}1.41 \\
(-0.15)\end{array}$ & $\begin{array}{r}0.00 \\
(0.00)\end{array}$ & $\begin{array}{l}-0.14 \\
(0.05)\end{array}$ & 7 \\
\hline
\end{tabular}

a $P$-value $<0.05$

${ }^{b} \mathrm{P}$-value $<0.001$ 
Table 4b. Indicator variable baseline (intercept) and annual change (slope) estimates by beach. Variable baseline of predicted 2008 value (Yearly Change). For all variables, a negative yearly change indicates improvement, 0 is no change, positive yearly change indicates degradation.

\begin{tabular}{|c|c|c|c|c|c|c|}
\hline Beach & $\begin{array}{r}\text { Mineral Soil } \\
\text { Exposure (\%) }\end{array}$ & Fire Rings & $\begin{array}{r}\text { Tree } \\
\text { Stumps }\end{array}$ & $\begin{array}{r}\text { Tree } \\
\text { Damage }\end{array}$ & $\begin{array}{r}\text { Root } \\
\text { Exposure }\end{array}$ & $\begin{array}{r}\text { \# of } \\
\text { Campsites }\end{array}$ \\
\hline Abra & $\begin{array}{r}67.53 \\
(-0.43)\end{array}$ & $\begin{array}{r}0.00 \\
(0.00)\end{array}$ & $\begin{array}{r}0.00 \\
(0.00)\end{array}$ & $\begin{array}{r}1.05 \\
(-0.17)\end{array}$ & $\begin{array}{r}0.84 \\
(-0.12)\end{array}$ & 7 \\
\hline Bear Cove & $\begin{array}{r}65.63 \\
(-2.96)\end{array}$ & $\begin{array}{r}0.11 \\
(0.00)\end{array}$ & $\begin{array}{r}0.69 \\
(-0.08)\end{array}$ & $\begin{array}{r}1.00 \\
(0.00)\end{array}$ & $\begin{array}{r}1.06 \\
(0.00)\end{array}$ & 6 \\
\hline Bulldog & $\begin{array}{r}82.62 \\
(-7.18)\end{array}$ & $\begin{array}{r}0.68 \\
(-0.10)\end{array}$ & $\begin{array}{r}0.00 \\
(0.00)\end{array}$ & $\begin{array}{r}1.53 \\
(-0.19)\end{array}$ & $\begin{array}{r}1.52 \\
(-0.25)^{\mathrm{a}}\end{array}$ & 5 \\
\hline Holgate North & $\begin{array}{r}70.66 \\
(-4.26)\end{array}$ & $\begin{array}{r}0.00 \\
(0.00)\end{array}$ & $\begin{array}{r}0.00 \\
(0.00)\end{array}$ & $\begin{array}{r}0.26 \\
(0.01)\end{array}$ & $\begin{array}{r}0.25 \\
(0.12)\end{array}$ & 6 \\
\hline Holgate South & $\begin{array}{r}124.29 \\
(-18.04)^{\mathrm{a}}\end{array}$ & $\begin{array}{r}0.00 \\
(0.00)\end{array}$ & $\begin{array}{r}0.00 \\
(0.00)\end{array}$ & $\begin{array}{r}1.30 \\
(-0.13)\end{array}$ & $\begin{array}{r}1.30 \\
(-0.13)\end{array}$ & 3 \\
\hline McMullen & $\begin{array}{l}98.00 \\
(0.00)\end{array}$ & $\begin{array}{r}0.07 \\
(0.13)\end{array}$ & $\begin{array}{r}0.00 \\
(0.00)\end{array}$ & $\begin{array}{r}0.09 \\
(0.00)\end{array}$ & $\begin{array}{r}0.00 \\
(0.00)\end{array}$ & 4 \\
\hline Northeastern & $\begin{array}{r}66.76 \\
(-3.54)^{a}\end{array}$ & $\begin{array}{r}0.13 \\
(0.00)\end{array}$ & $\begin{array}{l}-0.08 \\
(0.03)\end{array}$ & $\begin{array}{r}1.68 \\
(-0.23)^{\mathrm{a}}\end{array}$ & $\begin{array}{r}1.47 \\
(-0.19)^{b}\end{array}$ & 11 \\
\hline NW Landing & $\begin{array}{r}-0.13 \\
(8.88)\end{array}$ & $\begin{array}{r}0.75 \\
(-0.25)\end{array}$ & $\begin{array}{r}3.75 \\
(-1.25)\end{array}$ & $\begin{array}{r}0.75 \\
(0.25)\end{array}$ & $\begin{array}{r}0.75 \\
(0.25)\end{array}$ & 4 \\
\hline Pedersen & $\begin{array}{l}-11.08 \\
(7.50)^{a}\end{array}$ & $\begin{array}{r}0.29 \\
(0.01)\end{array}$ & $\begin{array}{r}-0.12 \\
(0.21)^{\mathrm{a}}\end{array}$ & $\begin{array}{r}0.84 \\
(0.11)^{\mathrm{a}}\end{array}$ & $\begin{array}{r}1.59 \\
(0.03)\end{array}$ & 13 \\
\hline Pocket Cove & $\begin{array}{r}93.92 \\
(-0.27)\end{array}$ & $\begin{array}{r}0.50 \\
(0.00)\end{array}$ & $\begin{array}{l}-0.35 \\
(0.17)\end{array}$ & $\begin{array}{r}-0.35 \\
(0.17)\end{array}$ & $\begin{array}{r}0.33 \\
(0.02)\end{array}$ & 2 \\
\hline Quicksand North & $\begin{array}{l}66.63 \\
(1.50)\end{array}$ & $\begin{array}{r}0.00 \\
(0.00)\end{array}$ & $\begin{array}{r}0.00 \\
(0.00)\end{array}$ & $\begin{array}{r}1.00 \\
(0.00)\end{array}$ & $\begin{array}{r}1.00 \\
(0.00)\end{array}$ & 4 \\
\hline Quicksand South & $\begin{array}{r}87.32 \\
(-3.34)\end{array}$ & $\begin{array}{r}0.00 \\
(0.00)\end{array}$ & $\begin{array}{r}0.00 \\
(0.00)\end{array}$ & $\begin{array}{r}1.36 \\
(-0.07)\end{array}$ & $\begin{array}{r}0.64 \\
(0.07)\end{array}$ & 7 \\
\hline Southwestern & $\begin{array}{l}95.50 \\
(0.00)\end{array}$ & $\begin{array}{r}0.00 \\
(0.00)\end{array}$ & $\begin{array}{r}0.00 \\
(0.00)\end{array}$ & $\begin{array}{r}1.58 \\
(-0.24)^{\mathrm{a}}\end{array}$ & $\begin{array}{r}1.58 \\
(-0.24)^{a}\end{array}$ & 6 \\
\hline Verdant & $\begin{array}{r}94.72 \\
(-0.60)\end{array}$ & $\begin{array}{r}0.51 \\
(-0.05)\end{array}$ & $\begin{array}{r}0.38 \\
(-0.01)\end{array}$ & $\begin{array}{r}1.22 \\
(-0.07)\end{array}$ & $\begin{array}{r}0.69 \\
(0.06)\end{array}$ & 7 \\
\hline
\end{tabular}

\footnotetext{
a P-value $<0.05$

${ }^{b}$ P-value $<0.001$
}

At the beach level, by summing all ranked values for each variable Bulldog, Holgate South and North, and Quicksand South recovered the most. NW Landing experienced the most degradation, followed by Pedersen and McMullen (Table 5). This was the same pattern for fully recovered campsites. NW Landing was the only beach to experience, on average, an increase in area size. 
While all remaining beaches had a reduction in campsite area, on average, campsites on Bear Cove, Bulldog, Holgate North and South, Northeastern, Pedersen, Pocket Cove, Quicksand South, Sunlight, and Verdant had the most significant area decreases (Figure 3). Sunlight beach was not included in this ranked analysis at the beach level because only one campsite was sampled more than once over the course of the sampling period. Beaches in Aialik Bay had the most recovering campsites.

Northwestern Fjord had less recovery and was the location for the most new sites found at the end of the study period. Bulldog beach was ranked the highest in terms of recovery but is the only beach sampled in Resurrection Bay. While we were able to rank the amount of change at each sampling level, change was still somewhat marginal for most variables. Change values for each indicator variable can be found by campsite and by region in the Appendix.

Table 5. Ranks of all indicator variables' change by beach.

\begin{tabular}{lr}
\hline Beach & Change Rank $^{\mathbf{a}}$ \\
\hline Bulldog & 1 \\
Holgate South & 2 \\
Holgate North & 3 \\
Quicksand South & 4 \\
Verdant & 5 \\
Bear Cove & 6 \\
Northeastern & 7 \\
Abra & 8 \\
Pocket Cove & 9 \\
Southwestern & 10 \\
Quicksand North & 11 \\
McMullen & 12 \\
Pedersen & 13 \\
NW Landing & 14 \\
\hline
\end{tabular}

a Change ranks are based on the amount of change occurring for each variable and their sum. By beach, each variable is ranked from most recovery to most degradation and all variable rank scores are summed. 


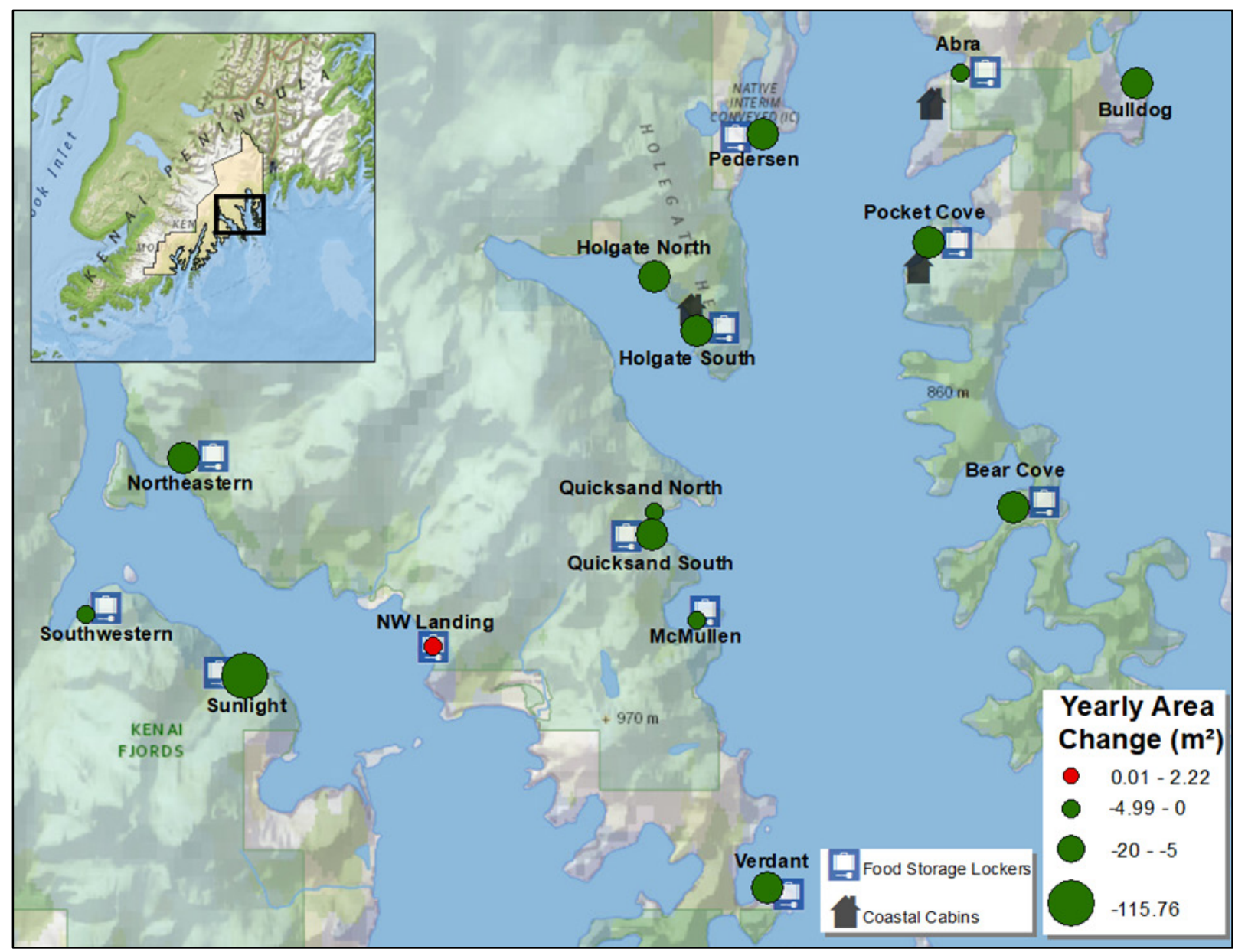

Figure 3. Yearly campsite area change by beach. Red campsite areas (NW Landing) depict area increases. Larger green circles depict greater area reductions.

\section{Ecological Variable Change}

Campsite area, tree damage, and root exposure are the most sensitive to change of all indicator variables. The data for this analysis includes the 70 campsites with at least two observation sample periods and no missing data. The exploratory principal component analysis revealed five factors that explained $64.6 \%$ of the variation in the change data. Campsite area, tree damage, and root exposure influence the first factor the most and account for $16.3 \%$ of the variance. The second factor explains $13.9 \%$ of the variability and includes variables: condition class, mineral soil exposure, trails, and trash (Table 6). For ease of interpretation, factor loadings between -0.4 and 0.4 are not listed. Factor loadings are reported as positive or negative because change occurs in either direction. 
Table 6. Factor analysis of indicator variables' change of campsites in KEFJ.

\begin{tabular}{lccccc}
\hline & \multicolumn{5}{c}{ Rotated Factor Loadings } \\
\cline { 2 - 6 } Variable & Factor $\mathbf{1}$ & Factor 2 & Factor 3 & Factor 4 & Factor 5 \\
\hline Campsite Area & -650 & - & - & - & - \\
Condition Class & - & .755 & - & - & - \\
Vegetation Cover Loss & - & - & -.708 & - & - \\
Mineral Soil Exposure & - & .462 & - & - & - \\
Tent Rocks & - & - & - & -.769 & - \\
Trails & - & .708 & - & - & - \\
Fire Rings & - & - & .764 & - & - \\
Tree Stumps & - & - & .768 & - & - \\
Tree Damage & .887 & - & - & - & - \\
Ghost Tree Damage & - & - & - & - & .926 \\
Root Exposure & .851 & - & - & - & - \\
Trash & - & .654 & - & - & - \\
Human Waste & - & - & - & .788 & - \\
Cumulative Variation Explanation (Percent) & 16.3 & 30.2 & 43.9 & 56.2 & 64.6 \\
\hline
\end{tabular}

a Principal components extraction results with varimax rotation. Factors loadings above 0.4 are presented for ease of interpretation. $\mathrm{N}=70$.

The ordination visualizes which campsites are changing differently from other campsites, based on the variables that loaded the highest in factor one and two. There appears to be no pattern of a specific beach experiencing more exaggerated change than others (Figure 4). Campsite 66, the only campsite on Sunlight beach with more than one year of data, is varying from the clustering of campsites for the factor one variables. This particular campsite did experience a decrease in area size between sampling periods. Where a majority of campsites were observed to have a $10 \mathrm{~m}^{2}$ or less difference in area size per year, campsite 66 decreased in size by $115.76 \mathrm{~m}^{2}$ annually. Additionally, most campsites do not exhibit much change in tree damage or root exposure from year to year, but for this site, severity for both variables increased. Campsite 47 also presented itself as an outlier in the ordination plot. This site stood out as different from other sites because of its annual $10.25 \mathrm{~m}^{2}$ area growth, where the majority of sites were declining in size. Similar to campsite 66, this site's change in tree damage and root exposure changes drastically from year to year. For most sites, if there was a change in these variables, it is incremental. Unlike campsite 66, campsite 47 had severe tree damage and root exposure in the first year of sampling and improved by the final sample (Tables A1a and A1b in Appendix A). 


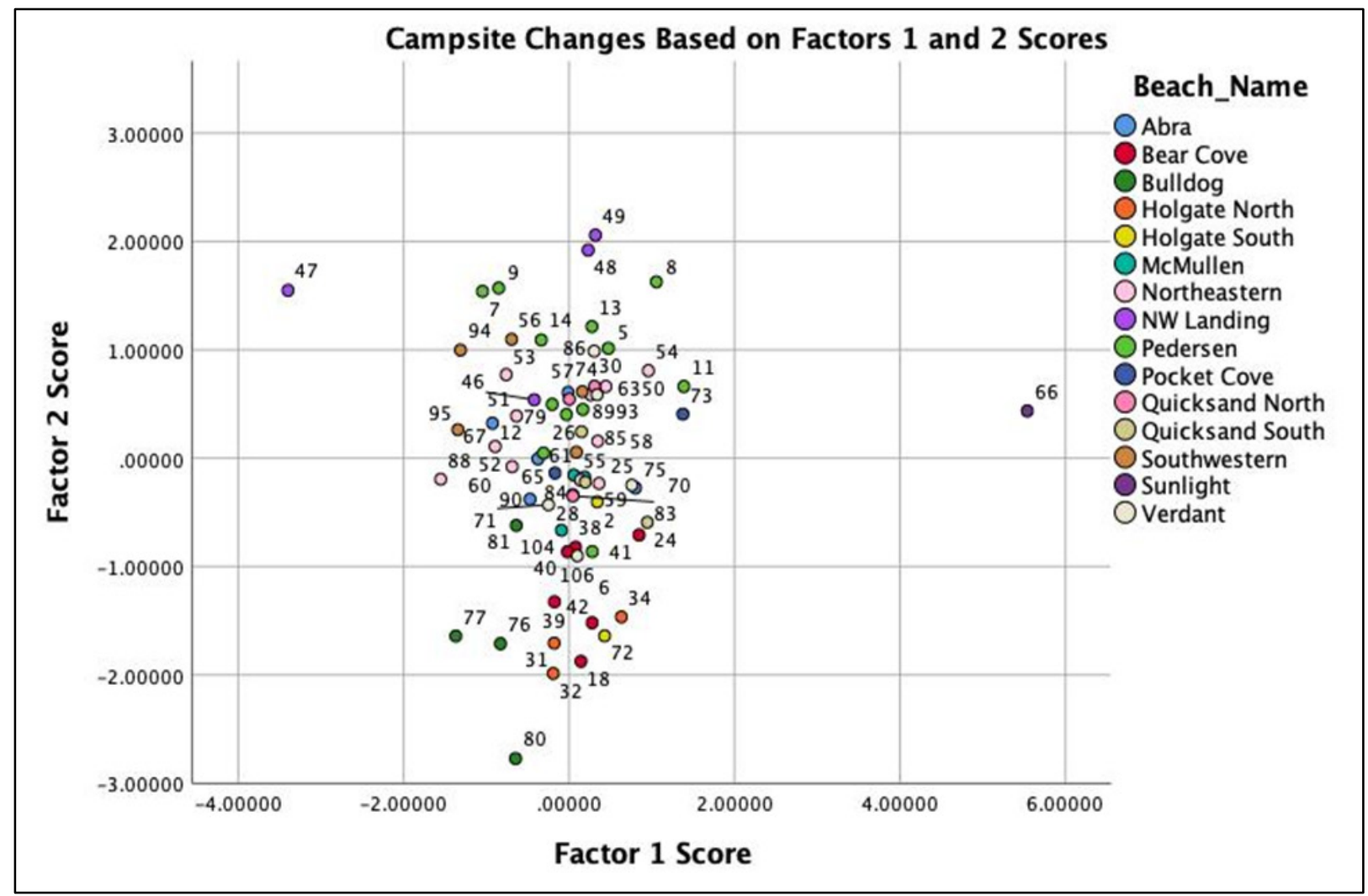

Figure 4. Scatterplot of campsites based on amount of change of variables in Factors 1 and 2. Factor 1: Area, Tree Damage, and Root Exposure. Factor 2: Condition Class, Mineral Soil Exposure, Trails, and Trash.

Factor two scores indicated some patterns by beach as all campsites on Bear Cove and Bulldog beach fell below zero on the $\mathrm{Y}$ axis while all of NW Landing and the majority of Pedersen campsites were above zero. This indicates there was a greater magnitude of change at these beaches for the variables condition class, mineral soil exposure, trails, and trash. Bear Cove and Bulldog campsites recovered more for all variables, while NW Landing and Pedersen campsites were degrading. This is evidenced when comparing Bulldog campsite 80 to NW Landing campsites 48 and 49. Campsite 80 improves by one condition class rating and decreases mineral soil exposure by $25 \%$ annually, ultimately recovering by the end of sampling. Both campsites 48 and 49 degraded by one condition class rating and gained a trail each year. Mineral soil exposure and trash observations remained static (Tables Ala and A1b in Appendix).

\section{Optimizing the Monitoring Protocol}

\section{Redundant Ecological Variables}

The data for this analysis included all 186 observations from the 101 campsites. Since this was the cleaned raw data, it included each sample made from all sampling periods for each campsite. Six equations were created to explain $74.1 \%$ of the variation in the data (Table 7). Variables loading similarly on the same factor accounted for similar characteristics in the data set. By eliminating one of the variables per factor, most of the variance could still be identified with less measurements. For 
example, factor two was most influenced by vegetation cover loss, mineral soil exposure, and tent rocks. A future protocol might suggest only measuring vegetation cover loss instead of mineral soil exposure, because it is easier to properly identify vegetation than it is mineral soil exposure with limited training. The elimination of the excessive variable measurement would still account for most of the variation in the data. Additionally, these results also suggest that removing trash, campsite area, and tent rocks as measured variables would yield a new protocol that would still capture 53.7\% of the variance in the data.

Table 7. Factor analysis of indicator variables' campsites in KEFJ.

\begin{tabular}{lcccccc}
\hline & \multicolumn{5}{c}{ Rotated Factor Loadings $^{\mathbf{a}}$} \\
\cline { 2 - 6 } Site Attribute & Factor 1 & Factor 2 & Factor 3 & Factor 4 & Factor 5 & Factor 6 \\
\hline Campsite Area & - & - & - & - & .799 & - \\
Vegetation Cover Loss & - & .841 & - & - & - & - \\
Mineral Soil Exposure & - & .890 & - & - & - & - \\
Tent Rocks & - & .437 & - & - & .643 & - \\
Trails & - & - & - & .655 & - & - \\
Fire Rings & - & - & - & .756 & - & - \\
Tree Stumps & - & - & - & .502 & - & - \\
Ghost Tree Stumps & - & - & .858 & - & - & - \\
Tree Damage & .940 & - & - & - & - & - \\
Ghost Tree Damage & - & - & .760 & - & - & - \\
Root Exposure & .935 & - & - & - & - & - \\
Trash & - & - & - & - & - & .882 \\
Cumulative Variation Explanation (Percent) & 16.6 & 31.5 & 42.7 & 53.7 & 64.0 & 74.1 \\
\hline
\end{tabular}

a Principal components extraction results with varimax rotation. Factors loadings above 0.4 are presented for ease of interpretation. $\mathrm{N}=186$.

\section{Sampling Interval}

To determine a more appropriate sampling interval, paired Student's T-tests were run to compare the value of the first sample of the measured variable to the last and test for a significant change (Tables $8 \mathrm{a}$ and $8 \mathrm{~b}$ ). Our results indicated very little observable change occurred within one year. Significant changes did occur after at least two years. For the categorical variables root exposure, trash, and human waste there was no change detected between sampling years therefore, they were not included in Table 8b. Because ghost tree stumps are evidence of the 1964 Good Friday earthquake, the number of stumps at a campsite should not change as the stumps remain stationary. For this reason, the change in the number of ghost tree stumps was not evaluated, as there was no change. However, in campsites with ghost trees present, the level of damage to them was evaluated because this 
variable serves as an assessment of human impact when the damage can clearly be identified as human caused. Increased signs of damage such as scratches or cuts provided evidence of increased or irresponsible use.

Table 8a. Continuous indicator variable change significance by sampling interval.

\begin{tabular}{|c|c|c|c|c|}
\hline Continuous Variable & Interval & Mean Difference & $p$-value & $\mathbf{N}$ \\
\hline \multirow[t]{4}{*}{ Area $\left(m^{2}\right)$} & 1 Year & -18.638 & 0.384 & 6 \\
\hline & 2 Years* & -10.695 & $<0.001$ & 30 \\
\hline & 3 Years $^{*}$ & -20.96 & $<0.001$ & 36 \\
\hline & 4 Years & -64.452 & 0.078 & 6 \\
\hline \multirow[t]{4}{*}{ Condition Class } & 1 Year & -0.429 & 0.120 & 7 \\
\hline & 2 Years* & 0.786 & $<0.001$ & 28 \\
\hline & 3 Years* & 0.686 & $<0.001$ & 35 \\
\hline & 4 Years* $^{*}$ & 2.364 & $<0.001$ & 11 \\
\hline \multirow[t]{4}{*}{ Vegetation Loss } & 1 Year & 26.112 & 0.232 & 9 \\
\hline & 2 Years & -6.994 & 0.142 & 25 \\
\hline & 3 Years & -9.911 & 0.057 & 32 \\
\hline & 4 Years & 0.500 & 0.965 & 12 \\
\hline \multirow[t]{4}{*}{ Mineral Soil Exposure } & 1 Year & -1.857 & 0.736 & 7 \\
\hline & 2 Years & -5.614 & 0.297 & 22 \\
\hline & 3 Years & 3.455 & 0.462 & 33 \\
\hline & 4 Years & 14.500 & 0.287 & 8 \\
\hline \multirow[t]{4}{*}{ Tent Rocks } & 1 Year & 0.143 & 0.928 & 7 \\
\hline & 2 Years* & 2.815 & 0.024 & 27 \\
\hline & 3 Years* & 1.280 & 0.042 & 34 \\
\hline & 4 Years & 3.100 & 0.451 & 10 \\
\hline \multirow[t]{4}{*}{ Trails } & 1 Year* & -0.571 & 0.030 & 7 \\
\hline & 2 Years & 0.179 & 0.510 & 28 \\
\hline & 3 Years & -0.400 & 0.124 & 35 \\
\hline & 4 Years* & 1.400 & 0.022 & 15 \\
\hline
\end{tabular}

* Significant p-value $<0.05$ 
Table 8a (continued). Continuous indicator variable change significance by sampling interval.

\begin{tabular}{llrrr}
\hline Continuous Variable & Interval & Mean Difference & p-value & $\mathbf{N}$ \\
\hline Fire Rings & 1 Year & 0.143 & 0.356 & 7 \\
& 2 Years & -0.072 & 0.161 & 28 \\
& 3 Years & 0.057 & 0.624 & 35 \\
& 4 Years & -0.133 & 0.164 & 15 \\
Tree Stumps & 1 Year & 0.714 & 0.253 & 7 \\
& 2 Years & 0 & 1.000 & 28 \\
& 3 Years & -0.229 & 0.147 & 35 \\
& 4 Years & 0.133 & 0.719 & 15 \\
\hline
\end{tabular}

* Significant $p$-value $<0.05$

Table 8b. Categorical indicator variable change significance by sampling interval.

\begin{tabular}{llrrr}
\hline Categorical Variable & Interval & $\chi^{\mathbf{2}}$ & p-value & $\mathbf{~ N}$ \\
\hline Tree Damage & 1 Year & 3.080 & 0.2144 & 7 \\
& 2 Years & 16.741 & 0.002 & 24 \\
& 3 Years & 2.954 & 0.566 & 33 \\
& 4 Years $^{*}$ & 11.123 & $<0.001$ & 6 \\
Ghost Tree Damage & 1 Year $^{*}$ & $\mathrm{~N} / \mathrm{A}$ & $\mathrm{N} / \mathrm{A}$ & 0 \\
& 2 Years $^{*}$ & 6.412 & 0.041 & 11 \\
& 3 Years $^{*}$ & 11.074 & 0.004 & 9 \\
& 4 Years $^{*}$ & 5.799 & 0.055 & 8 \\
\hline
\end{tabular}

* Significant $p$-value $<0.05$

\section{Ellipse Area Measurement}

In 2012, campsite areas measured with the radial transect method were, on average, $20.58 \mathrm{~m}^{2}$. On average, the same campsites measured using the ellipse estimation method were larger by a small margin at $21.02 \mathrm{~m}^{2}$ (Table 9). Radial transect and estimated area measurements were strongly and positively correlated $(\mathrm{r}=0.971, \mathrm{p}<0.001)$. There was no significant difference between radial transect and estimated area measurements $\left(\mathrm{t}_{53}=-0.93, \mathrm{p}=0.357\right)$. On average, radial transect area measurements were $0.45 \mathrm{~m}^{2}$ smaller than estimated measurements (95\% CI [-1.42, 0.52], Table 10). A simple linear regression analysis supported these results, explaining $94 \%$ of the variation across the two measures (Figure 5). 
Table 9. Descriptive statistics of different campsite area measurement methods. $\mathrm{N}=54$.

\begin{tabular}{lrr}
\hline Parameter & Mean & $\begin{array}{r}\text { Standard } \\
\text { Deviation }\end{array}$ \\
\hline Radial Area $\left(\mathrm{m}^{2}\right)$ & 20.58 & 14.66 \\
Estimated Area $\left(\mathrm{m}^{2}\right)$ & 21.02 & 14.57 \\
\hline
\end{tabular}

Table 10. Paired samples correlations of radial versus estimated campsite area measurement methods. $\mathrm{N}=54$.

\begin{tabular}{lrrrrrrr}
\hline Correlation & Mean & $\begin{array}{r}\text { Standard } \\
\text { Deviation }\end{array}$ & $\begin{array}{r}\text { Lower } \\
\mathbf{( 9 5 \% )}\end{array}$ & $\begin{array}{r}\text { Upper } \\
\mathbf{( 9 5 \% )}\end{array}$ & $\mathbf{t}$ & df & $\begin{array}{r}\text { Significance } \\
(\mathbf{2} \text {-tailed) }\end{array}$ \\
\hline Radial - Estimated & -0.45 & 3.55 & -1.42 & 0.52 & -0.93 & 53 & 0.357 \\
\hline
\end{tabular}

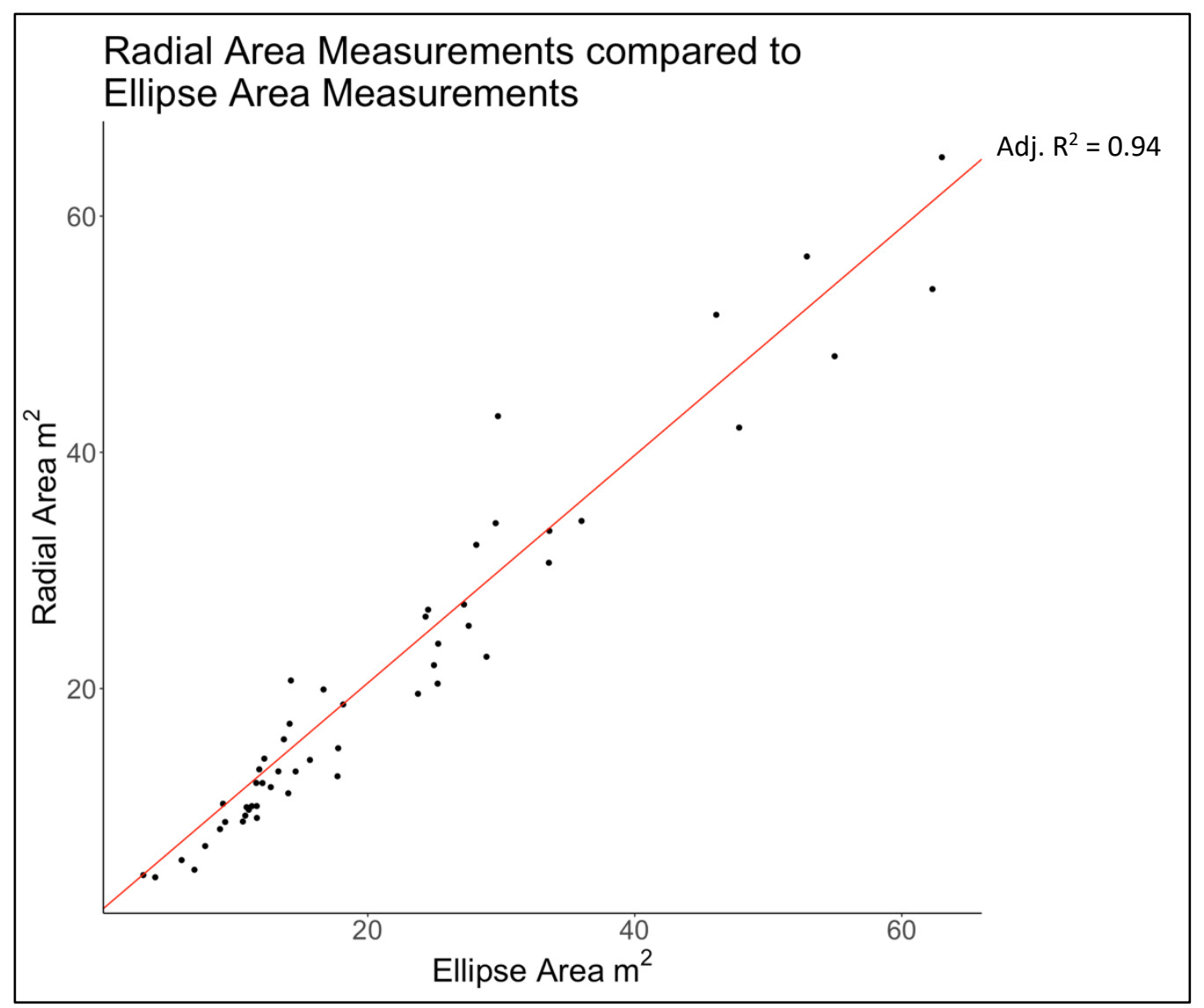

Figure 5. Simple linear regression model comparing radial area measurements to ellipse estimated area measurements. Regression line $p$-value $<0.01, R^{2}=0.94$. 


\section{Discussion}

This assessment of campsite changes and the efficiency of current monitoring procedures is an important step in determining best natural resource management practices. Visitors notice ecological impacts on campsites (Farrell et al., 2001). Results from monitoring the conditions of visitor use areas provides the biophysical evidence to bridge the gap of what visitors find acceptable in terms of ecological impact and the reality of the condition they are in (D'Antonio et al., 2013; Goonan et al., 2012). Additionally, results from these assessments provide land managers evidence to justify restoration strategies if determined necessary. Confining visitors to certain designated camping areas (Reid \& Marion, 2004), dispersing visitors to different areas (Cole et al., 2008), and closing campsites completely for restoration (Cole \& Hall, 1992) are proven management strategies that have protected the natural resource (Hammitt et al., 2015).

Based on the results of our investigation, for our first objective, we have concluded that the majority of sites indicated recovery or remained static, but there was no spatial pattern in changing campsites at the individual or beach level. However, some spatial patterns were seen by park region. Resurrection Bay exhibited the most recovery, but only consisted of Bulldog beach. Given that Bulldog beach is exposed to high surf and weather from the Gulf of Alaska it is more dangerous to land a vessel there and may suggest fewer visitation levels. NW Landing beach, located in Northwestern Fjord, was a region of some concern. It was the only beach where campsites, on average, increased in size and variables were degrading more than recovering. Pedersen and McMullen beaches had slightly more degrading tendencies than other beaches as well and should continue to be monitored because of the new sites located in the later years of sampling. The same could be said for Abra, Sunlight, Southwestern, and Northeastern beaches. These are areas where new sites were discovered in the last two years of complete sampling assessments and there was little evidence of full recovery on older campsites. In comparison to the work by Twardock et al. (2010) in Prince William Sound, overall KEFJ campsites exhibited more recovery but appear to experience more degradation in terms of mineral soil exposure. While Twardock et al. (2010) presented findings that visitors were using multiple locations with less intensity, our results suggest a change in the overall pattern of use intensity. Visitors appear to be camping in completely different beach locations over time, allowing some beaches to recover while focusing use in other locations. Bulldog may have once been a popular destination, but Northwestern Fjord and areas of Aialik Bay seem to experience more use currently.

Our second conclusion, is that while campsite area did change and is an important measure of campsite impact (Monz \& Twardock, 2010; Cole \& Hall, 1992), it did not account for much of the variation in the data (Table 7). These mixed results indicate that area change occurs in some places and remains static in others. The precise nature of conducting a radial transect area measurement allowed for determining more sensitive changes in area (Table 6), even though most changes in area were quite small. This suggests that perhaps future monitoring protocols may not require the precision that radial transect measurements offer. While exact campsite areas may be helpful to capture sensitive changes in the data, they can be quite cumbersome in the field. A revised protocol might include a more rapid area measurements such as our suggested ellipse area calculation. With 
no significant difference in the areas calculated from the radial transect method compared to the ellipse estimation and with $94 \%$ of the variance accounted for, the ellipse measurements still offer accurate area measurements with just slightly less precision. In the field, the campsite boundary would still be established, however only the length of the major and minor axis would be measured. With this type of measurement, managers will still be able to identify areas of concern and determine sites that are increasing in size at an alarming rate.

Finally, our results suggest a few modifications to the current rapid and complete assessment (Monz et al., 2011) techniques are necessary. With very little change occurring in one year and by campsite, change seems to occur more broadly by only a subset of campsites, and after at least two years.

Based on the paired Student's T-test and Pearson Chi-Square results, suitable monitoring assessments could be completed at a three to five-year sampling interval. Simultaneously considering all our statistical analysis approaches, the high priority variables would include: tree damage, vegetation cover on and offsite, tent rocks, trails, condition class ratings, ghost tree damage, and campsite area. Mineral soil exposure, vegetation cover loss, and root exposure all loaded fairly high in the principal component analysis and accounted for most of the variance in the data. As these variables were correlated, vegetation cover loss was chosen as the most effective indicator variable. Identifying percent vegetation cover on the campsite and at a control plot requires less training than identifying percent mineral soil exposure accurately. Mineral soil exposure can be a complicated variable to measure. It requires correctly identifying mineral soil substances as opposed to the more commonly seen organic soil layer. Root exposure was thought to be less universally applicable because a tree would need to be present on the campsite. Properly identifying an appropriate control plot to compare to the amount of vegetation found on the campsite is imperative to the vegetation cover loss variable. Technicians should be trained on locating control plots within five meters of the campsite's perimeter with the same substrate type. Since the variables trash and human waste occurred so infrequently and except for severe cases, are fairly ephemeral, they do not need their own category to classify during each observation. However, they are important indicators of previous use and deviations from Leave No Trace principles and they should be noted if found. Campsite substrate and high tide line measurements should also be taken for future analysis purposes that may shed light on the causation of some campsite changes. Henceforth, our optimal monitoring protocol would replace the need for separate rapid and complete campsite assessments, opting for one streamlined comprehensive sampling program that provides a consistent set of variables measured each time. Campsite assessments would occur every three to five years and include: rapid campsite area measurements, tree damage (noting N/A if there are no trees), percent vegetation onsite and at a control plot, tent rock counts, trail counts, condition class ratings, ghost tree damage (noting N/A if there are no ghost trees), campsite substrate type, high tide measurements (meters), and noting any campsite abnormalities such as trash or human waste. Sampling intervals should be adjusted if large amounts of change are occurring or if visitor use levels change significantly. In these instances, a shorter interval period may be necessary.

A successful monitoring protocol inevitably comes down to feasibility. Some of the limitations in our study were brought on because not all campsites could be sampled more than twice in the five-year study period. Our variable model predictions would be more robust if each campsite had more 
samples. Additionally, because these are results from observations taken a decade ago, it might be in the best interest of the park to conduct another round of monitoring using the original protocol to compare to these results. If large scale changes have not occurred, the monitoring interval could be extended beyond the suggested three to five years. Important logistics to consider when determining an appropriate monitoring protocol depends on finances, time, and crew availability. Locating the metal pins used as the site identifiers seems to be the most time consuming part of the assessment process. The investment in sub-decimeter high accuracy GPS units could potentially mitigate this issue. By collecting center point data at each site with a higher degree of precision, returning to that exact location should be much easier in years to come. Considering the geographical location of Alaska and the effect of the magnetic field in KEFJ, a sub-decimeter level of GPS precision when relocating the campsites center point pin may not be achievable. With this in mind, having photographs of the campsites with the center points clearly identified with permanent landmarks in the photographs may cut down on the search time for the center point. Efforts to relocate the metal center points with a high accuracy GPS unit and reference photos should not exceed five to seven minutes. A new center point using a technician's best judgement should be created if the search time exceeds seven minutes. More thoughtfully organized record keeping protocols could also decrease assessment times. This would include providing drop down menus to select from a short list of options to reduce sampling times for variables such as substrate type, percent vegetation cover, and tree and ghost tree damage. Taking counts of most variables as opposed to ordinal ocular estimations for variables such as tent rocks and trails would also provide more precise results. Additionally, understanding intensity of use or tracking where visitors camp would improve this analysis. Future research might include providing visitors with a GPS unit to track their trip or asking visitors to document which beaches they camped on after returning from their trip (D'Antonio et al., 2013; Stamberger et al., 2018).

Results from research such as this, provide park managers the information they need to determine how best to mitigate recreation ecological damage and maintain a wilderness experience for visitors. This analysis has also garnered evidence to improve existing campsite monitoring protocols. Management suggestions might include: designating campsites, moving to a reservation system for use, or promoting camping use on more durable surfaces. Furthermore, we believe the ecological conclusions brought forth in this report can be extended to public land campsites, off a road system, in temperate coastal rainforests. Campsites in British Columbia, Canada and the Pacific Northwest region of the United States would likely prove suitable environments to test the suggested optimized protocol. 


\section{Conclusions}

While patterns in campsites were hard to discern, looking more broadly at the park region level, beaches in Resurrection (Bulldog) and Aialik Bay (Holgate North and South and Quicksand South) recovered more than beaches in Northwestern Fjord (NW Landing and Sunlight) of KEFJ. Future research would include continued longitudinal monitoring of these campsites. To have data that spans decades and has consistent sampling periods would provide a more robust analysis. An alternative monitoring protocol would call for comprehensive sampling every three to five years focusing on: rapid campsite area measurements, tree damage, vegetation cover onsite and at a control plot, tent rock counts, trail counts, condition class ratings, and ghost tree damage. Research such as this should also expand to other locations. Conducting this study in areas with similar biomes could corroborate and support these results or provide new insight into ecological impacts of backcountry camping. 


\section{Literature Cited}

Arredondo, J. R., Meadema, F. P., \& Wimpey, J. F. 2018. Modeling areal measures of campsite impacts on the Appalachian National Scenic Trail to enhance ecological sustainability. 26. https://doi.org/10.1016/j.jenvman.2020.111693

Boggs, K., Klein, S. C., Flagstad, L., Boucher, T., Grunblatt, J., \& Koltun, B. 2008. Landcover classes, ecosystems and plant associations of Kenai Fjords National Park. Natural Resource Technical Report NPS/KEFJ/NRTR—2008/136. National Park Service, Fort Collins, Colorado.

Brame, R., \& Cole, D. 2011. Soft paths: Enjoying the wilderness without harming it (4 ${ }^{\text {th }}$ ed.). Stackpole Books.

Cole, D. N. 1995a. Experimental trampling of vegetation. I. Relationship between trampling intensity and vegetation response. Applied Ecology, 32, 203-214. https://doi.org/10.2307/2404429

Cole, D. N. 1995b. Experimental trampling of vegetation. II. Predictors of resistance and resilience. Applied Ecology, 32, 215-224. https://doi.org/10.2307/2404430

Cole, D. N., Foti, P., \& Brown, M. 2008. Twenty years of change on campsites in the backcountry of Grand Canyon National Park. Environmental Management. 41(6): 959-970. https://doi.org/10.1007/s00267-008-9087-5

Cole, D. N., \& Hall, T. E. 1992. Trends in campsite condition: Eagle Cap Wilderness, Bob Marshall Wilderness, and Grand Canyon National Park. Res. Pap. INT-453 Ogden: UT: U.S. Department of Agriculture, Forest Service, Intermountain Research Station.

D’Antonio, A., Monz, C., Newman, P., Lawson, S., \& Taff, D. 2013. Enhancing the utility of visitor impact assessment in parks and protected areas: A combined social-ecological approach. Journal of Environmental Management, 124, 72-81. https://doi.org/10.1016/j.jenvman.2013.03.036

Farrell, T., Hall, T. E., \& White, D. D. 2001. Wilderness campers' perception and evaluation of campsite impacts. Journal of Leisure Research, 33(3), 229-250.

https://doi.org/10.1080/00222216.2001.11949939

Goonan, K. A., Monz, C., Manning, R. E., \& Anderson, L. E. 2012. Resource conditions and paddler standards for primitive campsites along Lake Champlain. Journal of Great Lakes Research, 38, 157-166. https://doi.org/10.1016/j.jglr.2011.05.010

Hammitt W. E., Cole, D. N., \& Monz, C. 2015. Wildland recreation: Ecology and management (3 ${ }^{\text {rd }}$ ed.). John Wiley \& Sons.

Harrison, X. A., Donaldson, L., Correa-Cano, M. E., Evans, J., Fisher, D. N., Goodwin, C. E. D., Robinson, B. S., Hodgson, D. J., \& Inger, R. 2018. A brief introduction to mixed effects modelling and multi-model inference in ecology. PeerJ, 6, e4794.

https://doi.org/10.7717/peerj.4794 
Leung, Y. F., \& Marion, J. L. 1999. Characterizing backcountry camping impacts in Great Smokey Mountains National Park, USA. Journal of Environmental Management, 57, 193-203.

Leung, Y. F., \& Marion, J. L. 2000. Recreation impacts and management in wilderness: A state-ofknowledge review. US Forest Service Proceedings 5, 27.

Machlis, G. E., \& Tichnell, D. L. 2019. The state of the world's parks: An international assessment for resource management, policy, and research. Routledge.

Manning, R. E. 2011 Studies in outdoor recreation: Search and research for satisfaction ( $3^{\text {rd }}$ ed.). Oregon State University Press.

Marion, J. L. 1995. Capabilities and management utility of recreation impact monitoring programs. Environmental Management, 19, 763-771. https://doi.org/10.1007/BF02471958

Monz, C., Klasner, F., Goonan, K. 2011. Coastal campsite monitoring protocol- Kenai Fjords National Park. National Park Service, Fort Collins, Colorado. 73.

Monz, C. A., Pickering, C. M., \& Hadwen, W. L. 2013. Recent advances in recreation ecology and the implications of different relationships between recreation use and ecological impacts. Frontiers in Ecology and the Environment, 11(8), 441-446. https://doi.org/10.1890/120358

Monz, C. A., \& Twardock, P. 2010. A classification of backcountry campsites in Prince William Sound, Alaska, USA. Journal of Environmental Management, 91(7), 1566-1572. https://doi.org/10.1016/j.jenvman.2010.02.030

Nagorski, S., Hood, E., Eckert, G., \& Pyare, S. 2010. Assessment of coastal water resources and watershed conditions: Kenai Fjords National Park. Natural Resource Report NPS/NRPC/WRD/NRR—2010/192. National Park Service, Fort Collins, Colorado.

National Park Service. 2018. Kenai Fjords: Plant communities. U.S. Department of the Interior. https://www.nps.gov/kefj/learn/nature/plant-communities.htm.

Newsome, D., S. A. Moore, \& R. K. Dowling. 2001. Natural area tourism: Ecology, impacts, and management. Channel View Books, Clevedon, UK.

Reid, S. E., \& Marion, J. L. 2004. Effectiveness of a confinement strategy for reducing campsite impacts in Shenandoah National Park. Environmental Conservation, 31(4), 274-282. https://doi.org/10.1017/S0376892904001602

Ritter, D. F. 1986. Process geomorphology ( $2^{\text {nd }}$ ed.). William C. Brown Publishers, Dubuque, IA.

Shrader-Frechette, K. S., \& McCoy, E. D. 1995. Natural landscapes, natural communities, and natural ecosystems. Forest \& Conservation History, 39(3), 138-142. https://doi.org/10.2307/3983518 
Stamberger, L., van Riper, C. J., Keller, R., Brownlee, M., \& Rose, J. 2018. A GPS tracking study of recreationists in an Alaskan protected area. Applied Geography, 93, 92-102. https://doi.org/10.1016/j.apgeog.2018.02.011

Twardock, P., Monz, C., Smith, M., \& Colt, S. 2010. Long-term changes in resource conditions on backcountry campsites in Prince William Sound, Alaska, USA. Northwest Science, 84(3), 223 232. https://doi.org/10.3955/046.084.0302 


\section{Appendix A. Annual Ecological Variable Changes by Campsite and Park Region}

Indicator variable baseline and annual change estimates are presented by campsite in Tables Ala and $\mathrm{A} 1 \mathrm{~b}$ and by park region in Tables $\mathrm{A} 2 \mathrm{a}$ and $\mathrm{A} 2 \mathrm{~b}$. 
Table A1a. Indicator variable baseline (intercept) and annual change (slope) estimates by campsite. Variable baseline of predicted 2008 value (Yearly Change). For all variables, a negative yearly change indicates improvement, 0 is no change, positive yearly change indicates degradation.

\begin{tabular}{|c|c|c|c|c|c|c|c|c|c|c|}
\hline Site & $\begin{array}{r}\text { Tag } \\
\text { Number }\end{array}$ & Area $\left(\mathrm{m}^{2}\right)$ & $\begin{array}{r}\text { Condition } \\
\text { Class }\end{array}$ & $\begin{array}{r}\text { Vegetation } \\
\text { Loss (\%) }\end{array}$ & $\begin{array}{r}\text { Tent } \\
\text { Rocks }\end{array}$ & Trails & $\begin{array}{r}\text { Ghost Tree } \\
\text { Damage }\end{array}$ & Trash & $\begin{array}{r}\text { Human } \\
\text { Waste }\end{array}$ & $\begin{array}{r}\text { Latitude }\left({ }^{\circ} \mathrm{N}\right. \\
\text { Longitude }\left({ }^{\circ} \mathrm{W}\right.\end{array}$ \\
\hline \multirow[t]{7}{*}{ Abra } & 67 & $\begin{array}{r}17.96 \\
(3.08)^{a}\end{array}$ & $\begin{array}{r}2.00 \\
(0.00)^{\mathrm{a}}\end{array}$ & $\begin{array}{l}-226.20 \\
(30.70)^{\mathrm{a}}\end{array}$ & $\begin{array}{r}16.00 \\
(0.00)^{\mathrm{a}}\end{array}$ & $\begin{array}{r}2.00 \\
(0.00)^{\mathrm{a}}\end{array}$ & $\begin{array}{r}1.00 \\
(0.00)^{\mathrm{a}}\end{array}$ & $\begin{array}{r}0.00 \\
(0.00)^{\mathrm{a}}\end{array}$ & $\begin{array}{r}0.00 \\
(0.00)^{\mathrm{a}}\end{array}$ & $\begin{array}{r}59.8945 \\
149.6438\end{array}$ \\
\hline & 68 & $\begin{array}{r}44.78 \\
(-4.87)^{\mathrm{a}}\end{array}$ & $\begin{array}{r}3.67 \\
(-0.33)^{\mathrm{a}}\end{array}$ & $\begin{array}{l}-23.60 \\
(0.00)^{\mathrm{a}}\end{array}$ & $\begin{array}{r}16.00 \\
(0.00)^{\mathrm{a}}\end{array}$ & $\begin{array}{r}4.00 \\
(0.00)^{\mathrm{a}}\end{array}$ & $\begin{array}{r}1.00 \\
(0.00)^{\mathrm{a}}\end{array}$ & $\begin{array}{r}0.00 \\
(0.00)^{\mathrm{a}}\end{array}$ & $\begin{array}{r}0.00 \\
(0.00)^{\mathrm{a}}\end{array}$ & $\begin{array}{r}59.8944 \\
149.644 C\end{array}$ \\
\hline & 69 & NA & $\begin{array}{r}1.00 \\
(1.00)^{\mathrm{a}}\end{array}$ & $\begin{array}{l}-34.23 \\
(9.55)^{\mathrm{a}}\end{array}$ & $\begin{array}{r}16.00 \\
(0.00)^{\mathrm{a}}\end{array}$ & $\begin{array}{r}-2.00 \\
(1.00)^{\mathrm{a}}\end{array}$ & $\begin{array}{r}1.00 \\
(0.00)^{\mathrm{a}}\end{array}$ & $\begin{array}{r}0.00 \\
(0.00)^{\mathrm{a}}\end{array}$ & $\begin{array}{r}0.00 \\
(0.00)^{\mathrm{a}}\end{array}$ & $\begin{array}{r}59.8942 \\
149.6447\end{array}$ \\
\hline & 74 & $\begin{array}{r}28.72 \\
(-4.15)\end{array}$ & $\begin{array}{r}2.57 \\
(-0.07)\end{array}$ & $\begin{array}{l}-69.11 \\
(12.22)\end{array}$ & $\begin{array}{r}13.00 \\
(-2.00)\end{array}$ & $\begin{array}{r}1.43 \\
(0.07)\end{array}$ & $\begin{array}{r}1.00 \\
(0.00)^{\mathrm{a}}\end{array}$ & $\begin{array}{r}0.00 \\
(0.00)^{\mathrm{a}}\end{array}$ & $\begin{array}{r}0.00 \\
(0.00)^{\mathrm{a}}\end{array}$ & $\begin{array}{r}59.8943 \\
149.6423\end{array}$ \\
\hline & 75 & $\begin{array}{r}42.61 \\
(-1.98)\end{array}$ & $\begin{array}{r}4.43 \\
(-0.43)\end{array}$ & $\begin{array}{r}4.27 \\
(-6.19)\end{array}$ & $\begin{array}{r}16.00 \\
(0.00)^{\mathrm{a}}\end{array}$ & $\begin{array}{r}2.57 \\
(-0.07)\end{array}$ & $\begin{array}{r}1.00 \\
(0.00)^{\mathrm{a}}\end{array}$ & $\begin{array}{r}0.00 \\
(0.00)^{\mathrm{a}}\end{array}$ & $\begin{array}{r}0.00 \\
(0.00)^{\mathrm{a}}\end{array}$ & $\begin{array}{r}59.8944 \\
149.6443\end{array}$ \\
\hline & 90 & $\begin{array}{r}24.47 \\
(-3.80)^{\mathrm{a}}\end{array}$ & $\begin{array}{r}2.00 \\
(0.00)^{a}\end{array}$ & $\begin{array}{l}-88.62 \\
(9.76)^{\mathrm{a}}\end{array}$ & $\begin{array}{r}24.25 \\
(-2.75)^{a}\end{array}$ & $\begin{array}{r}4.50 \\
(-0.50)^{a}\end{array}$ & $\begin{array}{r}1.00 \\
(0.00)^{\mathrm{a}}\end{array}$ & $\begin{array}{r}0.00 \\
(0.00)^{\mathrm{a}}\end{array}$ & $\begin{array}{r}0.00 \\
(0.00)^{\mathrm{a}}\end{array}$ & $\begin{array}{r}59.8944 \\
149.6425\end{array}$ \\
\hline & 91 & NA & $\begin{array}{r}4.50 \\
(-0.50)^{\mathrm{a}}\end{array}$ & $\begin{array}{l}-15.13 \\
(0.00)^{\mathrm{a}}\end{array}$ & $\begin{array}{r}24.25 \\
(-2.75)^{\mathrm{a}}\end{array}$ & $\begin{array}{r}1.00 \\
(0.00)^{\mathrm{a}}\end{array}$ & $\begin{array}{r}1.00 \\
(0.00)^{\mathrm{a}}\end{array}$ & $\begin{array}{r}0.00 \\
(0.00)^{\mathrm{a}}\end{array}$ & $\begin{array}{r}0.00 \\
(0.00)^{\mathrm{a}}\end{array}$ & $\begin{array}{r}59.8943 \\
149.6410\end{array}$ \\
\hline \multirow[t]{3}{*}{ Bear Cove } & 18 & $\begin{array}{r}67.50 \\
(-8.44)^{\mathrm{a}}\end{array}$ & $\begin{array}{r}4.83 \\
(-0.50)\end{array}$ & $\begin{array}{r}-30.04 \\
(5.16)\end{array}$ & $\begin{array}{r}6.38 \\
(0.37)\end{array}$ & $\begin{array}{r}4.50 \\
(-0.50)^{\mathrm{d}}\end{array}$ & $\begin{array}{r}1.25 \\
(0.25)^{\mathrm{a}}\end{array}$ & $\begin{array}{r}1.10 \\
(-0.25)^{a}\end{array}$ & $\begin{array}{r}0.00 \\
(0.00)^{\mathrm{a}}\end{array}$ & $\begin{array}{r}59.7907 \\
149.6167\end{array}$ \\
\hline & 38 & $\begin{array}{r}73.78 \\
(-9.42)^{a}\end{array}$ & $\begin{array}{r}4.83 \\
(-0.50)\end{array}$ & $\begin{array}{l}-7.69 \\
(0.00)\end{array}$ & $\begin{array}{l}14.92 \\
(0.25)\end{array}$ & $\begin{array}{r}2.58 \\
(0.25)\end{array}$ & $\begin{array}{c}1.67 \\
(0.00)^{\mathrm{a}}\end{array}$ & $\begin{array}{r}1.08 \\
(-0.25)^{\mathrm{a}}\end{array}$ & $\begin{array}{r}0.00 \\
(0.00)^{\mathrm{a}}\end{array}$ & $\begin{array}{r}59.7896 \\
149.6167\end{array}$ \\
\hline & 39 & $\begin{array}{r}48.86 \\
(-3.26)\end{array}$ & $\begin{array}{r}4.83 \\
(-0.50)\end{array}$ & $\begin{array}{r}-3.06 \\
(0.26)\end{array}$ & $\begin{array}{r}20.33 \\
(-3.00)\end{array}$ & $\begin{array}{r}4.20 \\
(-0.50)\end{array}$ & $\begin{array}{r}1.00 \\
(0.00)^{\mathrm{a}}\end{array}$ & $\begin{array}{r}1.75 \\
(-0.25)^{a}\end{array}$ & $\begin{array}{r}0.00 \\
(0.00)^{\mathrm{a}}\end{array}$ & $\begin{array}{r}59.7897 \\
149.6167\end{array}$ \\
\hline
\end{tabular}

a Sample size of 2. P-value is not attainable.

${ }^{b}$ Recovered campsite

c P-value $<0.05$

d $P$-value $<0.001$ 
Table A1a (continued). Indicator variable baseline (intercept) and annual change (slope) estimates by campsite. Variable baseline of predicted 2008 value (Yearly Change). For all variables, a negative yearly change indicates improvement, 0 is no change, positive yearly change indicates degradation.

\begin{tabular}{|c|c|c|c|c|c|c|c|c|c|c|}
\hline Site & $\begin{array}{r}\text { Tag } \\
\text { Number }\end{array}$ & Area $\left(m^{2}\right)$ & $\begin{array}{r}\text { Condition } \\
\text { Class }\end{array}$ & $\begin{array}{l}\text { Vegetation } \\
\text { Loss (\%) }\end{array}$ & $\begin{array}{r}\text { Tent } \\
\text { Rocks }\end{array}$ & Trails & $\begin{array}{r}\text { Ghost Tree } \\
\text { Damage }\end{array}$ & Trash & $\begin{array}{l}\text { Human } \\
\text { Waste }\end{array}$ & $\begin{array}{r}\text { Latitude }\left({ }^{\circ} \mathrm{N}\right) \\
\text { Longitude }\left({ }^{\circ} \mathrm{W}\right)\end{array}$ \\
\hline \multirow{6}{*}{$\begin{array}{l}\text { Bear Cove } \\
\text { (continued) }\end{array}$} & 40 & 61.55 & 3.42 & -44.10 & 7.67 & 5.17 & 0.92 & 1.08 & 0.00 & 59.7898 \\
\hline & & $(-10.30)^{a}$ & $(-0.25)$ & $(5.16)$ & $(-0.50)$ & $(-0.50)$ & $(0.25)^{a}$ & $(-0.25)^{a}$ & $(0.00)^{a}$ & 149.6167 \\
\hline & 41 & 287.99 & 3.42 & -62.12 & -1.00 & 2.92 & 1.67 & 1.08 & 0.00 & 59.7903 \\
\hline & & $(-46.28)^{a}$ & $(-0.25)$ & $(-0.99)$ & (3.50) & $(0.25)^{a}$ & $(0.00)^{a}$ & $(-0.25)^{a}$ & $(0.00)^{a}$ & 149.6166 \\
\hline & $42^{b}$ & 137.60 & 2.50 & -94.75 & 0.00 & 3.58 & 1.00 & 1.08 & 0.00 & 59.7905 \\
\hline & & $(-28.95)$ & $(-0.50)^{d}$ & $(0.00)$ & $(0.00)^{a}$ & $(-0.75)$ & $(0.00)^{\mathrm{a}}$ & $(-0.25)^{a}$ & $(0.00)^{a}$ & 149.6161 \\
\hline \multirow[t]{10}{*}{ Bulldog } & 76 & 57.00 & 4.43 & -39.58 & 20.71 & 3.86 & 1.00 & 1.86 & 0.00 & 59.8913 \\
\hline & & $(-3.10)$ & $(-0.43)$ & $(4.02)$ & $(-1.96)$ & $(-0.36)$ & $(0.00)^{a}$ & $(-0.36)^{a}$ & $(0.00)^{a}$ & 149.5580 \\
\hline & $77^{b}$ & 40.01 & 3.14 & 191.32 & 27.00 & 5.00 & 1.00 & 0.00 & 0.00 & 59.8914 \\
\hline & & $(-8.25)$ & $(-0.64)$ & $(-145.16)^{a}$ & $(-5.50)^{a}$ & $(-1.00)^{d}$ & $(0.00)^{a}$ & $(0.00)^{a}$ & $(0.00)^{a}$ & 149.5577 \\
\hline & 78 & NA & 3.00 & -20.04 & 0.00 & 0.00 & 1.00 & 0.00 & 0.00 & 59.8921 \\
\hline & & & $(-1.00)^{a}$ & $(-26.32)^{a}$ & $(0.00)^{a}$ & $(0.00)^{a}$ & $(0.00)^{a}$ & $(0.00)^{a}$ & $(0.00)^{a}$ & 149.5632 \\
\hline & $80^{b}$ & 120.67 & 5.57 & 18.75 & 13.00 & 0.29 & 1.57 & 1.86 & 0.00 & 59.8912 \\
\hline & & $(-24.12)^{a}$ & $(-1.07)$ & $(-20.95)^{c}$ & $(-2.00)$ & $(0.21)$ & $(-0.07)^{a}$ & $(-0.36)^{a}$ & $(0.00)^{a}$ & 149.5572 \\
\hline & 81 & 123.10 & 3.00 & -15.32 & 16.00 & -0.29 & 1.00 & 1.86 & 0.00 & 59.8913 \\
\hline & & $(-21.73)$ & $(0.00)$ & $(2.13)$ & $(0.00)^{a}$ & $(0.79)$ & $(0.00)^{a}$ & $(-0.36)^{a}$ & $(0.00)^{a}$ & 149.5572 \\
\hline
\end{tabular}

a Sample size of 2. P-value is not attainable.

${ }^{\mathrm{b}}$ Recovered campsite

c $P$-value $<0.05$

d $P$-value $<0.001$ 
Table A1a (continued). Indicator variable baseline (intercept) and annual change (slope) estimates by campsite. Variable baseline of predicted 2008 value (Yearly Change). For all variables, a negative yearly change indicates improvement, 0 is no change, positive yearly change indicates degradation.

\begin{tabular}{|c|c|c|c|c|c|c|c|c|c|c|}
\hline Site & $\begin{array}{r}\text { Tag } \\
\text { Number }\end{array}$ & Area $\left(\mathrm{m}^{2}\right)$ & $\begin{array}{r}\text { Condition } \\
\text { Class }\end{array}$ & $\begin{array}{r}\text { Vegetation } \\
\text { Loss (\%) }\end{array}$ & $\begin{array}{r}\text { Tent } \\
\text { Rocks }\end{array}$ & Trails & $\begin{array}{r}\text { Ghost Tree } \\
\text { Damage }\end{array}$ & Trash & $\begin{array}{l}\text { Human } \\
\text { Waste }\end{array}$ & $\begin{array}{r}\text { Latitude }\left({ }^{\circ} \mathrm{N}\right) \\
\text { Longitude }\left({ }^{\circ} \mathbf{W}\right)\end{array}$ \\
\hline \multirow[t]{12}{*}{ Holgate North } & $31^{b}$ & 28.23 & 3.92 & -52.64 & 28.09 & 2.50 & 1.00 & 1.08 & 0.00 & 59.8456 \\
\hline & & $(-5.65)^{a}$ & $(-0.75)$ & $(-6.68)^{a}$ & $(-6.25)$ & $(-0.50)^{d}$ & $(0.00)^{a}$ & $(-0.25)^{a}$ & $(0.00)^{a}$ & 149.7887 \\
\hline & 32 & 14.78 & 4.58 & 2.04 & 15.25 & 4.58 & 1.00 & 1.08 & 0.00 & 59.8456 \\
\hline & & $(-0.73)^{a}$ & $(-0.75)$ & $(-5.42)$ & $(-2.25)$ & $(-0.75)$ & $(0.00)^{a}$ & $(-0.25)^{a}$ & $(0.00)^{a}$ & 149.7889 \\
\hline & 33 & NA & 3.92 & -15.24 & 28.04 & 7.50 & 1.00 & 1.08 & 0.00 & 59.8455 \\
\hline & & & $(-0.75)$ & $(-5.67)$ & $(-3.63)$ & $(-1.50)$ & $(0.00)^{a}$ & $(-0.25)^{a}$ & $(0.00)^{a}$ & 149.7890 \\
\hline & $34^{b}$ & 79.52 & 5.00 & -15.51 & 12.75 & 2.50 & 1.00 & 1.08 & 0.00 & 59.8453 \\
\hline & & $(-15.90)^{a}$ & $(-1.00)^{d}$ & $(-8.10)^{a}$ & $(-0.75)^{a}$ & $(-0.50)^{d}$ & $(0.00)^{a}$ & $(-0.25)^{a}$ & $(0.00)^{a}$ & 149.7888 \\
\hline & $35^{b}$ & NA & 3.25 & -15.66 & 7.50 & 3.25 & 1.00 & 1.08 & 0.00 & 59.8454 \\
\hline & & & $(-0.75)$ & $(-27.78)^{a}$ & $(-1.50)^{a}$ & $(-0.75)$ & $(0.00)^{a}$ & $(-0.25)^{a}$ & $(0.00)^{a}$ & 149.7888 \\
\hline & 36 & NA & 3.50 & -52.64 & 14.50 & 5.50 & 2.50 & 1.50 & 0.00 & 59.8457 \\
\hline & & & $(-0.50)^{a}$ & $(-6.68)^{a}$ & $(0.50)^{a}$ & $(-1.50)^{a}$ & $(-0.50)^{\mathrm{a}}$ & $(-0.50)^{a}$ & $(0.00)^{a}$ & 149.7890 \\
\hline \multirow[t]{6}{*}{ Holgate South } & 2 & 87.38 & 4.50 & -59.05 & 10.50 & 4.50 & 1.00 & 0.00 & 0.00 & 59.8318 \\
\hline & & $(-16.48)$ & $(-0.50)^{a}$ & $(7.09)^{a}$ & $(0.00)^{a}$ & $(-0.50)^{a}$ & $(0.00)^{a}$ & $(0.00)^{a}$ & $(0.00)^{a}$ & 149.7688 \\
\hline & $64^{\mathrm{b}}$ & 32.46 & 5.00 & NA & 5.00 & 0.00 & 1.00 & 0.00 & 0.00 & 59.8365 \\
\hline & & $(-6.49)^{a}$ & $(-1.00)^{a}$ & & $(-1.00)^{a}$ & $(0.00)^{a}$ & $(0.00)^{a}$ & $(0.00)^{a}$ & $(0.00)^{a}$ & 149.7717 \\
\hline & $72^{\mathrm{b}}$ & 32.97 & 5.00 & 35.49 & 3.86 & 1.86 & 1.00 & 0.00 & 0.00 & 59.8380 \\
\hline & & $(-6.34)$ & $(-1.00)^{d}$ & $(-26.31)^{a}$ & $(-0.86)$ & $(-0.36)$ & $(0.00)^{a}$ & $(0.00)^{a}$ & $(0.00)^{a}$ & 149.7740 \\
\hline
\end{tabular}

a Sample size of 2. P-value is not attainable.

${ }^{b}$ Recovered campsite

c P-value $<0.05$

d $P$-value $<0.001$ 
Table A1a (continued). Indicator variable baseline (intercept) and annual change (slope) estimates by campsite. Variable baseline of predicted 2008 value (Yearly Change). For all variables, a negative yearly change indicates improvement, 0 is no change, positive yearly change indicates degradation.

\begin{tabular}{|c|c|c|c|c|c|c|c|c|c|c|}
\hline Site & $\begin{array}{r}\text { Tag } \\
\text { Number }\end{array}$ & Area $\left(\mathrm{m}^{2}\right)$ & $\begin{array}{r}\text { Condition } \\
\text { Class }\end{array}$ & $\begin{array}{r}\text { Vegetation } \\
\text { Loss (\%) }\end{array}$ & $\begin{array}{r}\text { Tent } \\
\text { Rocks }\end{array}$ & Trails & $\begin{array}{r}\text { Ghost Tree } \\
\text { Damage }\end{array}$ & Trash & $\begin{array}{l}\text { Human } \\
\text { Waste }\end{array}$ & $\begin{array}{r}\text { Latitude }\left({ }^{\circ} \mathrm{N}\right) \\
\text { Longitude }\left({ }^{\circ} \mathbf{W}\right)\end{array}$ \\
\hline \multirow[t]{8}{*}{ McMullen } & 84 & 35.23 & 2.00 & -71.04 & -0.25 & -0.50 & 1.33 & 1.08 & 0.00 & 59.7634 \\
\hline & & $(-4.45)^{a}$ & $(0.00)^{a}$ & $(0.00)$ & $(3.75)$ & $(0.50)^{d}$ & $(0.00)^{a}$ & $(-0.25)^{a}$ & $(0.00)^{a}$ & 149.7685 \\
\hline & 85 & -4.34 & -0.50 & -133.95 & -16.50 & -0.42 & 2.42 & 1.08 & 1.08 & 59.7635 \\
\hline & & $(2.68)^{a}$ & $(0.50)^{a}$ & $(20.97)$ & $(6.50)^{\mathrm{a}}$ & $(0.25)$ & $(-0.25)^{a}$ & $(-0.25)^{a}$ & $(-0.25)^{a}$ & 149.7684 \\
\hline & 104 & 47.34 & 2.00 & 23.00 & 16.00 & 2.00 & 2.75 & 1.08 & 0.00 & 59.7633 \\
\hline & & $(-6.48)^{a}$ & $(0.00)^{a}$ & $(-20.97)$ & $(0.00)^{a}$ & $(0.00)^{a}$ & $(-0.25)^{a}$ & $(-0.25)^{a}$ & $(0.00)^{a}$ & 149.7686 \\
\hline & 114 & NA & NA & -99.00 & NA & 1.25 & 1.00 & 1.25 & 0.00 & 59.7627 \\
\hline & & & & $(0.00)^{a}$ & & $(-0.25)^{a}$ & $(0.00)^{a}$ & $(-0.25)^{a}$ & $(0.00)^{a}$ & 149.7684 \\
\hline \multirow[t]{10}{*}{ Northeastern } & 50 & 414.34 & 2.57 & -89.53 & 16.00 & 0.43 & 1.00 & 0.00 & 0.00 & 59.8016 \\
\hline & & $(-57.82)$ & $(-0.07)$ & (2.01) & $(0.00)^{\mathrm{a}}$ & $(0.57)$ & $(0.00)^{a}$ & $(0.00)^{a}$ & $(0.00)^{a}$ & 150.0138 \\
\hline & 51 & 35.44 & 2.00 & -144.91 & 0.86 & 1.57 & 1.00 & 0.00 & 0.00 & 59.8018 \\
\hline & & $(-5.68)$ & $(0.00)^{a}$ & $(10.20)$ & $(2.14)$ & $(-0.07)$ & $(0.00)^{a}$ & $(0.00)^{a}$ & $(0.00)^{a}$ & 150.0138 \\
\hline & 52 & 44.46 & 2.57 & -80.05 & 7.29 & 2.57 & 1.00 & 0.00 & 0.00 & 59.8015 \\
\hline & & $(-6.87)^{\mathrm{c}}$ & $(-0.07)$ & $(7.98)$ & $(-0.54)$ & $(-0.07)$ & $(0.00)^{a}$ & $(0.00)^{a}$ & $(0.00)^{a}$ & 150.0137 \\
\hline & 53 & 54.66 & 0.71 & -144.91 & 5.57 & 0.14 & 1.00 & 0.00 & 0.00 & 59.8015 \\
\hline & & $(-10.30)$ & $(0.29)$ & $(10.20)$ & $(-1.07)$ & $(0.36)$ & $(0.00)^{a}$ & $(0.00)^{a}$ & $(0.00)^{a}$ & 150.0135 \\
\hline & 54 & 191.49 & 3.00 & -134.31 & 16.00 & 1.36 & 1.00 & 0.00 & 0.00 & 59.8007 \\
\hline & & $(-7.87)$ & $(0.00)$ & $(20.25)$ & $(0.00)^{a}$ & $(0.36)$ & $(0.00)^{a}$ & $(0.00)^{a}$ & $(0.00)^{a}$ & 150.0126 \\
\hline
\end{tabular}

a Sample size of 2. P-value is not attainable.

${ }^{b}$ Recovered campsite

c P-value $<0.05$

d P-value $<0.001$ 
Table A1a (continued). Indicator variable baseline (intercept) and annual change (slope) estimates by campsite. Variable baseline of predicted 2008 value (Yearly Change). For all variables, a negative yearly change indicates improvement, 0 is no change, positive yearly change indicates degradation.

\begin{tabular}{|c|c|c|c|c|c|c|c|c|c|c|}
\hline Site & $\begin{array}{r}\text { Tag } \\
\text { Number }\end{array}$ & Area $\left(\mathrm{m}^{2}\right)$ & $\begin{array}{r}\text { Condition } \\
\text { Class }\end{array}$ & $\begin{array}{r}\text { Vegetation } \\
\text { Loss (\%) }\end{array}$ & $\begin{array}{r}\text { Tent } \\
\text { Rocks }\end{array}$ & Trails & $\begin{array}{r}\text { Ghost Tree } \\
\text { Damage }\end{array}$ & Trash & $\begin{array}{r}\text { Human } \\
\text { Waste }\end{array}$ & $\begin{array}{r}\text { Latitude }\left({ }^{\circ} \mathrm{N}\right) \\
\text { Longitude }\left({ }^{\circ} \mathrm{W}\right)\end{array}$ \\
\hline \multirow{12}{*}{$\begin{array}{l}\text { Northeastern } \\
\text { (continued) }\end{array}$} & 55 & 47.19 & 3.90 & 6.39 & 10.50 & 3.00 & 1.00 & 0.00 & 0.00 & 59.8009 \\
\hline & & $(-1.02)^{a}$ & $(-0.40)$ & $(-20.07)$ & $(0.00)^{a}$ & $(0.00)^{a}$ & $(0.00)^{a}$ & $(0.00)^{a}$ & $(0.00)^{a}$ & 150.0126 \\
\hline & 58 & 70.25 & 3.89 & -5.98 & 17.57 & -0.14 & 1.00 & 0.00 & 0.00 & 59.8004 \\
\hline & & $(-8.72)$ & $(-0.36)$ & $(-2.84)$ & $(-1.57)$ & $(0.64)$ & $(0.00)^{a}$ & $(0.00)^{a}$ & $(0.00)^{a}$ & 150.0123 \\
\hline & 59 & 71.83 & 3.67 & -136.04 & 16.00 & 4.00 & 1.00 & 0.00 & 0.00 & 59.8006 \\
\hline & & $(-1.37)^{a}$ & $(-0.33)^{a}$ & $(18.52)^{a}$ & $(0.00)^{a}$ & $(0.00)^{a}$ & $(0.00)^{a}$ & $(0.00)^{a}$ & $(0.00)^{a}$ & 150.0125 \\
\hline & 60 & 38.76 & 3.00 & -28.13 & 17.65 & 4.70 & 1.00 & 0.00 & 0.00 & 59.8005 \\
\hline & & $(4.46)$ & $(0.00)^{a}$ & (1.67) & $(-1.65)$ & $(-0.20)$ & $(0.00)^{a}$ & $(0.00)^{a}$ & $(0.00)^{a}$ & 150.0128 \\
\hline & 88 & 29.25 & 4.50 & -91.11 & 26.25 & 3.00 & 1.00 & 0.00 & 0.00 & 59.8004 \\
\hline & & $(-1.94)^{a}$ & $(-0.50)^{a}$ & $(17.11)^{\mathrm{a}}$ & $(-5.25)^{a}$ & $(0.00)^{a}$ & $(0.00)^{a}$ & $(0.00)^{a}$ & $(0.00)^{a}$ & 150.0125 \\
\hline & 89 & 39.17 & 4.67 & -10.46 & 25.50 & -1.33 & 1.00 & 0.00 & 0.00 & 59.8004 \\
\hline & & $(-5.20)^{a}$ & $(-0.50)$ & $(1.83)$ & $(-5.25)$ & $(1.00)$ & $(0.00)^{a}$ & $(0.00)^{a}$ & $(0.00)^{a}$ & 150.0124 \\
\hline \multirow[t]{8}{*}{ NW Landing } & 46 & 17.47 & 3.00 & -262.74 & 27.00 & 3.00 & 1.00 & 0.00 & 0.00 & 59.7563 \\
\hline & & $(-1.04)^{a}$ & $(0.00)^{a}$ & $(81.87)^{a}$ & $(-5.50)^{a}$ & $(0.00)^{a}$ & $(0.00)^{a}$ & $(0.00)^{a}$ & $(0.00)^{a}$ & 149.8944 \\
\hline & 47 & 10.89 & 0.00 & 101.68 & -12.00 & 1.00 & 1.00 & 0.00 & 0.00 & 59.7564 \\
\hline & & $(10.25)^{a}$ & $(1.00)^{a}$ & $(-62.64)^{a}$ & $(7.50)^{a}$ & $(1.00)^{a}$ & $(0.00)^{a}$ & $(0.00)^{a}$ & $(0.00)^{a}$ & 149.8945 \\
\hline & 48 & 12.78 & -1.00 & -20.04 & 9.00 & 0.00 & 1.00 & 0.00 & 0.00 & 59.7565 \\
\hline & & $(0.09)^{a}$ & $(1.00)^{a}$ & $(-26.32)^{a}$ & $(-3.00)^{a}$ & $(1.00)^{a}$ & $(0.00)^{a}$ & $(0.00)^{a}$ & $(0.00)^{a}$ & 149.8945 \\
\hline & 49 & 18.45 & -1.00 & -99.00 & 3.00 & 0.00 & 1.00 & 0.00 & 0.00 & 59.7566 \\
\hline & & $(-0.41)^{a}$ & $(1.00)^{a}$ & $(0.00)^{a}$ & $(0.00)^{a}$ & $(1.00)^{a}$ & $(0.00)^{a}$ & $(0.00)^{a}$ & $(0.00)^{a}$ & 149.8945 \\
\hline
\end{tabular}

a Sample size of 2. P-value is not attainable.

${ }^{b}$ Recovered campsite

c P-value $<0.05$

d P-value $<0.001$ 
Table A1a (continued). Indicator variable baseline (intercept) and annual change (slope) estimates by campsite. Variable baseline of predicted 2008 value (Yearly Change). For all variables, a negative yearly change indicates improvement, 0 is no change, positive yearly change indicates degradation.

\begin{tabular}{|c|c|c|c|c|c|c|c|c|c|c|}
\hline Site & $\begin{array}{r}\text { Tag } \\
\text { Number }\end{array}$ & Area $\left(m^{2}\right)$ & $\begin{array}{r}\text { Condition } \\
\text { Class }\end{array}$ & $\begin{array}{r}\text { Vegetation } \\
\text { Loss (\%) }\end{array}$ & $\begin{array}{r}\text { Tent } \\
\text { Rocks }\end{array}$ & Trails & $\begin{array}{r}\text { Ghost Tree } \\
\text { Damage }\end{array}$ & Trash & $\begin{array}{r}\text { Human } \\
\text { Waste }\end{array}$ & $\begin{array}{l}\text { Latitude }\left({ }^{\circ} \mathrm{N}\right) \\
\text { Longitude }\left({ }^{\circ} \mathrm{W}\right)\end{array}$ \\
\hline \multirow[t]{20}{*}{ Pedersen } & 5 & 303.71 & 3.00 & -27.94 & 7.29 & 5.86 & 1.00 & 0.00 & 0.00 & 59.8796 \\
\hline & & $(-61.65)$ & $(0.00)$ & $(1.64)$ & $(-0.54)$ & $(0.14)$ & $(0.00)^{a}$ & $(0.00)^{a}$ & $(0.00)^{a}$ & 149.7369 \\
\hline & 6 & 149.98 & 2.57 & -64.02 & 3.00 & 7.43 & 2.71 & 0.00 & 0.00 & 59.8797 \\
\hline & & $(-28.05)$ & $(-0.07)$ & $(-5.65)$ & $(0.00)$ & $(-1.43)$ & $(-0.21)^{a}$ & $(0.00)^{a}$ & $(0.00)^{a}$ & 149.7370 \\
\hline & 7 & 11.71 & 3.00 & -37.78 & 21.75 & 4.50 & 1.00 & 0.00 & 0.00 & 59.8798 \\
\hline & & (2.39) & $(0.00)^{a}$ & $(0.00)^{a}$ & $(-3.75)^{a}$ & $(0.50)^{a}$ & $(0.00)^{a}$ & $(0.00)^{a}$ & $(0.00)^{a}$ & 149.7367 \\
\hline & 8 & 15.94 & 3.00 & -34.17 & 7.50 & -0.50 & 1.00 & 0.00 & 0.00 & 59.8797 \\
\hline & & $(-0.32)$ & $(0.00)^{a}$ & $(6.45)^{a}$ & $(-1.50)^{a}$ & $(1.50)^{a}$ & $(0.00)^{a}$ & $(0.00)^{a}$ & $(0.00)^{a}$ & 149.7366 \\
\hline & 9 & 43.24 & 3.00 & -29.29 & 21.75 & 2.00 & 1.00 & 0.00 & 0.00 & 59.8797 \\
\hline & & $(-0.06)$ & $(0.00)^{a}$ & $(-2.83)^{a}$ & $(-3.75)^{a}$ & $(1.00)^{a}$ & $(0.00)^{a}$ & $(0.00)^{a}$ & $(0.00)^{a}$ & 149.7366 \\
\hline & $11^{b}$ & 100.25 & 0.00 & -99.00 & -4.50 & 0.00 & -0.50 & 0.00 & 0.00 & 59.8796 \\
\hline & & $(-22.28)$ & $(0.00)^{a}$ & $(0.00)^{a}$ & $(1.50)^{\mathrm{a}}$ & $(0.00)^{a}$ & $(0.50)^{a}$ & $(0.00)^{a}$ & $(0.00)^{a}$ & 149.7373 \\
\hline & 12 & 20.84 & 2.86 & -86.24 & 0.00 & 2.14 & 2.86 & 0.00 & 0.00 & 59.8798 \\
\hline & & $(-1.09)$ & $(-0.36)$ & $(0.00)$ & $(0.00)^{a}$ & $(0.36)$ & $(-0.36)^{a}$ & $(0.00)^{a}$ & $(0.00)^{a}$ & 149.7370 \\
\hline & 13 & 17.10 & 2.00 & -57.03 & 5.00 & -1.00 & 1.00 & 0.00 & 0.00 & 59.8797 \\
\hline & & $(-1.80)^{\mathrm{a}}$ & $(0.00)^{a}$ & $(-3.13)^{a}$ & $(-1.00)^{a}$ & $(1.00)^{a}$ & $(0.00)^{a}$ & $(0.00)^{a}$ & $(0.00)^{a}$ & 149.7369 \\
\hline & 14 & 13.61 & 2.00 & -55.24 & 5.57 & 0.29 & 1.00 & 0.00 & 0.00 & 59.8799 \\
\hline & & $(-0.78)$ & $(0.00)^{a}$ & $(-3.35)$ & $(-1.07)$ & $(0.71)$ & $(0.00)^{a}$ & $(0.00)^{a}$ & $(0.00)^{a}$ & 149.7367 \\
\hline & 16 & 37.19 & 4.00 & -15.81 & 0.14 & 5.86 & 2.86 & 0.00 & 0.00 & 59.8795 \\
\hline & & $(-1.77)$ & $(0.00)^{a}$ & $(-0.16)$ & (2.61) & $(-0.36)$ & $(-0.36)^{a}$ & $(0.00)^{a}$ & $(0.00)^{a}$ & 149.7371 \\
\hline
\end{tabular}

a Sample size of 2. P-value is not attainable.

${ }^{b}$ Recovered campsite

c P-value $<0.05$

d P-value $<0.001$ 
Table A1a (continued). Indicator variable baseline (intercept) and annual change (slope) estimates by campsite. Variable baseline of predicted 2008 value (Yearly Change). For all variables, a negative yearly change indicates improvement, 0 is no change, positive yearly change indicates degradation.

\begin{tabular}{|c|c|c|c|c|c|c|c|c|c|c|}
\hline Site & $\begin{array}{r}\text { Tag } \\
\text { Number }\end{array}$ & Area $\left(m^{2}\right)$ & $\begin{array}{r}\text { Condition } \\
\text { Class }\end{array}$ & $\begin{array}{r}\text { Vegetation } \\
\text { Loss (\%) }\end{array}$ & $\begin{array}{r}\text { Tent } \\
\text { Rocks }\end{array}$ & Trails & $\begin{array}{c}\text { Ghost Tree } \\
\text { Damage }\end{array}$ & Trash & $\begin{array}{l}\text { Human } \\
\text { Waste }\end{array}$ & $\begin{array}{r}\text { Latitude }\left({ }^{\circ} \mathrm{N}\right) \\
\text { Longitude }\left({ }^{\circ} \mathrm{W}\right)\end{array}$ \\
\hline \multirow{6}{*}{$\begin{array}{l}\text { Pedersen } \\
\text { (continued) }\end{array}$} & 79 & 15.61 & 0.43 & -99.00 & 1.29 & 0.43 & 1.00 & 0.00 & 0.00 & 59.8799 \\
\hline & & $(-2.32)^{a}$ & $(0.07)$ & $(0.00)$ & $(0.21)$ & $(0.07)$ & $(0.00)^{a}$ & $(0.00)^{a}$ & $(0.00)^{\mathrm{a}}$ & 149.7364 \\
\hline & 92 & NA & 3.00 & -93.07 & 3.00 & 0.00 & 1.00 & 0.00 & 0.00 & 59.8795 \\
\hline & & & $(0.00)^{a}$ & $(9.93)^{a}$ & $(0.00)^{a}$ & $(1.00)^{a}$ & $(0.00)^{a}$ & $(0.00)^{a}$ & $(0.00)^{a}$ & 149.7368 \\
\hline & 93 & 9.90 & 2.00 & -116.54 & -4.50 & 3.00 & 1.00 & 0.00 & 0.00 & 59.8795 \\
\hline & & $(0.34)^{a}$ & $(0.00)^{a}$ & $(14.62)^{a}$ & $(1.50)^{a}$ & $(0.00)^{a}$ & $(0.00)^{\mathrm{a}}$ & $(0.00)^{a}$ & $(0.00)^{a}$ & 149.7367 \\
\hline \multirow[t]{4}{*}{ Pocket Cove } & 65 & 52.92 & 5.29 & -18.90 & 10.50 & -1.14 & 2.86 & 0.00 & 0.00 & 59.8533 \\
\hline & & $(-7.03)$ & $(-0.79)$ & (3.77) & $(0.00)$ & $(0.64)$ & $(-0.36)^{a}$ & $(0.00)^{a}$ & $(0.00)^{a}$ & 149.6577 \\
\hline & 73 & 73.81 & 2.67 & -64.34 & 19.67 & 0.00 & 1.00 & 0.00 & 0.00 & 59.8535 \\
\hline & & $(-11.95)^{a}$ & $(-0.33)^{a}$ & $(12.27)^{\mathrm{a}}$ & $(-1.83)^{a}$ & $(0.00)^{a}$ & $(0.00)^{a}$ & $(0.00)^{a}$ & $(0.00)^{a}$ & 149.6577 \\
\hline \multirow{8}{*}{$\begin{array}{l}\text { Quicksand } \\
\text { North }\end{array}$} & 27 & 34.98 & 3.00 & -38.37 & 3.00 & 3.00 & 1.00 & 0.00 & 0.00 & 59.7886 \\
\hline & & $(3.77)^{\mathrm{a}}$ & $(0.00)^{a}$ & $(7.08)^{a}$ & $(0.00)^{a}$ & $(0.00)^{a}$ & $(0.00)^{\mathrm{a}}$ & $(0.00)^{a}$ & $(0.00)^{a}$ & 149.7885 \\
\hline & 28 & 54.92 & 4.50 & -20.13 & -8.25 & 3.50 & 3.50 & 0.00 & 0.00 & 59.7888 \\
\hline & & $(-9.24)^{a}$ & $(-0.50)^{a}$ & $(1.00)^{a}$ & $(3.75)^{a}$ & $(-0.50)^{a}$ & $(-0.50)^{a}$ & $(0.00)^{a}$ & $(0.00)^{a}$ & 149.7884 \\
\hline & 30 & 7.96 & 2.00 & -96.60 & 0.00 & 1.50 & 1.00 & 0.00 & 0.00 & 59.7880 \\
\hline & & $(-0.06)^{a}$ & $(0.00)^{a}$ & $(18.94)^{a}$ & $(0.00)^{a}$ & $(0.50)^{a}$ & $(0.00)^{a}$ & $(0.00)^{a}$ & $(0.00)^{a}$ & 149.7894 \\
\hline & 83 & 25.41 & 3.00 & -5.58 & 7.50 & 6.00 & 1.00 & 0.00 & 0.00 & 59.7884 \\
\hline & & $(-2.49)^{\mathrm{a}}$ & $(0.00)^{a}$ & $(0.00)^{a}$ & $(-1.50)^{a}$ & $(-1.00)^{a}$ & $(0.00)^{a}$ & $(0.00)^{a}$ & $(0.00)^{\mathrm{a}}$ & 149.7889 \\
\hline
\end{tabular}

a Sample size of 2. P-value is not attainable.

${ }^{b}$ Recovered campsite

c P-value $<0.05$

d $P$-value $<0.001$ 
Table A1a (continued). Indicator variable baseline (intercept) and annual change (slope) estimates by campsite. Variable baseline of predicted 2008 value (Yearly Change). For all variables, a negative yearly change indicates improvement, 0 is no change, positive yearly change indicates degradation.

\begin{tabular}{|c|c|c|c|c|c|c|c|c|c|c|}
\hline Site & $\begin{array}{r}\text { Tag } \\
\text { Number }\end{array}$ & Area $\left(m^{2}\right)$ & $\begin{array}{r}\text { Condition } \\
\text { Class }\end{array}$ & $\begin{array}{r}\text { Vegetation } \\
\text { Loss (\%) }\end{array}$ & $\begin{array}{r}\text { Tent } \\
\text { Rocks }\end{array}$ & Trails & $\begin{array}{r}\text { Ghost Tree } \\
\text { Damage }\end{array}$ & Trash & $\begin{array}{c}\text { Human } \\
\text { Waste }\end{array}$ & $\begin{array}{r}\text { Latitude }\left({ }^{\circ} \mathrm{N}\right) \\
\text { Longitude }\left({ }^{\circ} \mathrm{W}\right)\end{array}$ \\
\hline \multirow{14}{*}{$\begin{array}{l}\text { Quicksand } \\
\text { South }\end{array}$} & \multirow[t]{2}{*}{$19^{b}$} & 107.77 & 5.00 & \multirow[t]{2}{*}{ NA } & \multirow[t]{2}{*}{ NA } & 3.50 & 3.50 & 0.00 & 0.00 & 59.7841 \\
\hline & & $(-21.55)^{a}$ & $(-1.00)^{a}$ & & & $(-0.50)^{a}$ & $(-0.50)^{a}$ & $(0.00)^{a}$ & $(0.00)^{a}$ & 149.7897 \\
\hline & \multirow[t]{2}{*}{$21^{b}$} & 36.34 & 2.50 & \multirow[t]{2}{*}{ NA } & 7.50 & 1.00 & -0.50 & 0.00 & 0.00 & 59.7840 \\
\hline & & $(-7.27)^{a}$ & $(-0.50)^{a}$ & & $(-1.50)^{a}$ & $(0.00)^{a}$ & $(0.50)^{a}$ & $(0.00)^{a}$ & $(0.00)^{a}$ & 149.7896 \\
\hline & \multirow[t]{2}{*}{22} & NA & 7.50 & \multirow[t]{2}{*}{ NA } & 7.50 & 4.50 & 3.50 & 0.00 & 0.00 & 59.7835 \\
\hline & & & $(-1.50)^{a}$ & & $(-1.50)^{a}$ & $(-0.50)$ & $(-0.50)^{a}$ & $(0.00)^{a}$ & $(0.00)^{a}$ & 149.7895 \\
\hline & \multirow[t]{2}{*}{$23^{b}$} & 25.13 & 5.00 & \multirow[t]{2}{*}{ NA } & 26.25 & 5.00 & 3.50 & 0.00 & 0.00 & 59.7831 \\
\hline & & $(-5.03)^{a}$ & $(-1.00)^{a}$ & & $(-5.25)^{a}$ & $(-1.00)^{a}$ & $(-0.50)^{a}$ & $(0.00)^{a}$ & $(0.00)^{a}$ & 149.7891 \\
\hline & \multirow[t]{2}{*}{24} & 24.46 & 3.50 & -72.68 & 21.75 & 3.50 & 1.00 & 0.00 & 0.00 & 59.7827 \\
\hline & & $(-3.04)^{a}$ & $(-0.50)^{a}$ & $(0.00)^{a}$ & $(-3.75)^{a}$ & $(-0.50)^{a}$ & $(0.00)^{a}$ & $(0.00)^{a}$ & $(0.00)^{a}$ & 149.7889 \\
\hline & \multirow[t]{2}{*}{25} & 68.70 & 4.50 & -95.02 & 3.00 & 4.50 & 1.00 & 0.00 & 0.00 & 59.7826 \\
\hline & & $(-11.17)^{a}$ & $(-0.50)^{a}$ & $(18.41)^{\mathrm{a}}$ & $(0.00)^{a}$ & $(-0.50)^{a}$ & $(0.00)^{a}$ & $(0.00)^{a}$ & $(0.00)^{a}$ & 149.7889 \\
\hline & \multirow[t]{2}{*}{26} & 27.50 & 4.50 & -33.31 & 24.25 & 0.50 & 1.00 & 0.00 & 0.00 & 59.7828 \\
\hline & & $(-0.74)^{a}$ & $(-0.50)^{a}$ & $(3.24)^{a}$ & $(-2.75)^{a}$ & $(0.50)^{a}$ & $(0.00)^{a}$ & $(0.00)^{a}$ & $(0.00)^{a}$ & 149.7891 \\
\hline \multirow[t]{6}{*}{ Southwestern } & \multirow[t]{2}{*}{56} & 9.32 & 1.36 & -22.95 & 16.00 & -1.29 & 1.00 & 0.00 & 0.00 & 59.7641 \\
\hline & & $(1.45)$ & $(0.36)$ & $(4.34)$ & $(0.00)^{a}$ & $(0.71)$ & $(0.00)^{a}$ & $(0.00)^{a}$ & $(0.00)^{a}$ & 150.0605 \\
\hline & \multirow[t]{2}{*}{57} & 10.85 & 2.00 & -15.13 & 16.00 & -0.67 & 1.00 & 0.00 & 0.00 & 59.7643 \\
\hline & & $(0.46)$ & $(0.00)^{a}$ & $(0.00)^{a}$ & $(0.00)^{a}$ & $(0.33)^{a}$ & $(0.00)^{a}$ & $(0.00)^{a}$ & $(0.00)^{a}$ & 150.0605 \\
\hline & \multirow[t]{2}{*}{61} & 8.28 & 3.67 & -23.24 & 19.67 & 1.00 & 1.00 & 0.00 & 0.00 & 59.7640 \\
\hline & & $(0.71)$ & $(-0.33)^{a}$ & $(4.05)^{a}$ & $(-1.83)^{a}$ & $(0.00)^{a}$ & $(0.00)^{a}$ & $(0.00)^{a}$ & $(0.00)^{a}$ & 150.0608 \\
\hline
\end{tabular}

a Sample size of 2. P-value is not attainable.

${ }^{b}$ Recovered campsite

c $P$-value $<0.05$

d P-value $<0.001$ 
Table A1a (continued). Indicator variable baseline (intercept) and annual change (slope) estimates by campsite. Variable baseline of predicted 2008 value (Yearly Change). For all variables, a negative yearly change indicates improvement, 0 is no change, positive yearly change indicates degradation.

\begin{tabular}{|c|c|c|c|c|c|c|c|c|c|c|}
\hline Site & $\begin{array}{r}\text { Tag } \\
\text { Number }\end{array}$ & Area $\left(\mathrm{m}^{2}\right)$ & $\begin{array}{r}\text { Condition } \\
\text { Class }\end{array}$ & $\begin{array}{r}\text { Vegetation } \\
\text { Loss (\%) }\end{array}$ & $\begin{array}{r}\text { Tent } \\
\text { Rocks }\end{array}$ & Trails & $\begin{array}{r}\text { Ghost Tree } \\
\text { Damage }\end{array}$ & Trash & $\begin{array}{l}\text { Human } \\
\text { Waste }\end{array}$ & $\begin{array}{r}\text { Latitude }\left({ }^{\circ} \mathrm{N}\right) \\
\text { Longitude }\left({ }^{\circ} \mathrm{W}\right)\end{array}$ \\
\hline \multirow{6}{*}{$\begin{array}{l}\text { Southwestern } \\
\text { (continued) }\end{array}$} & 94 & 18.50 & 0.50 & -29.46 & 2.25 & 0.50 & 1.00 & 0.00 & 0.00 & 59.7644 \\
\hline & & $(-1.93)^{a}$ & $(0.50)^{a}$ & $(4.78)^{a}$ & $(2.75)^{a}$ & $(0.50)^{a}$ & $(0.00)^{a}$ & $(0.00)^{a}$ & $(0.00)^{\mathrm{a}}$ & 150.0602 \\
\hline & 95 & 19.32 & 2.00 & -187.82 & 2.25 & 2.00 & 1.00 & 0.00 & 0.00 & 59.7644 \\
\hline & & $(-2.38)^{a}$ & $(0.00)^{a}$ & $(29.61)^{a}$ & $(2.75)^{a}$ & $(0.00)^{a}$ & $(0.00)^{a}$ & $(0.00)^{a}$ & $(0.00)^{a}$ & 150.0604 \\
\hline & $107^{b}$ & 39.51 & NA & NA & NA & NA & NA & NA & NA & 59.7643 \\
\hline & & $(-7.90)^{\mathrm{a}}$ & & & & & & & & 150.0606 \\
\hline \multirow[t]{2}{*}{ Sunlight } & 66 & 355.90 & 1.00 & 20.04 & 10.50 & 0.00 & 1.00 & 0.00 & 0.00 & 59.7493 \\
\hline & & $(-115.76)^{a}$ & $(0.00)^{a}$ & $(-39.68)^{a}$ & $(0.00)^{a}$ & $(0.00)^{a}$ & $(0.00)^{a}$ & $(0.00)^{a}$ & $(0.00)^{a}$ & 149.9845 \\
\hline \multirow[t]{10}{*}{ Verdant } & 63 & 95.52 & 2.14 & -9.67 & 20.71 & -1.71 & 2.86 & 0.00 & 0.00 & 59.6986 \\
\hline & & $(-13.58)$ & $(-0.14)$ & $(-0.68)$ & $(-1.96)$ & $(0.71)$ & $(-0.36)^{a}$ & $(0.00)^{a}$ & $(0.00)^{a}$ & 149.7329 \\
\hline & 70 & 54.92 & 4.71 & -59.30 & 10.50 & 3.86 & 1.29 & 0.00 & 0.00 & 59.6968 \\
\hline & & $(-10.08)^{c}$ & $(-0.71)$ & (11.91) & $(0.00)$ & $(-0.36)$ & $(0.21)^{a}$ & $(0.00)^{a}$ & $(0.00)^{a}$ & 149.7387 \\
\hline & 71 & 72.43 & 4.71 & -10.96 & 16.93 & 1.57 & 1.00 & 0.00 & 0.00 & 59.6967 \\
\hline & & $(-10.35)$ & $(-0.71)$ & $(1.35)$ & $(-2.68)$ & $(-0.07)$ & $(0.00)^{a}$ & $(0.00)^{a}$ & $(0.00)^{a}$ & 149.7388 \\
\hline & 86 & 38.41 & 1.57 & -15.13 & 13.64 & -1.71 & 1.43 & 0.00 & 0.00 & 59.6986 \\
\hline & & $(-4.54)^{a}$ & $(-0.07)$ & $(0.00)$ & $(-0.39)$ & $(0.71)$ & $(0.07)^{a}$ & $(0.00)^{a}$ & $(0.00)^{a}$ & 149.7331 \\
\hline & $87^{b}$ & 31.52 & 5.00 & NA & 26.25 & 5.00 & 1.00 & 0.00 & 0.00 & 59.6982 \\
\hline & & $(-6.30)^{a}$ & $(-1.00)^{a}$ & & $(-5.25)^{a}$ & $(-1.00)^{a}$ & $(0.00)^{a}$ & $(0.00)^{a}$ & $(0.00)^{\mathrm{a}}$ & 149.7340 \\
\hline
\end{tabular}

a Sample size of 2. P-value is not attainable.

${ }^{\mathrm{b}}$ Recovered campsite

c P-value $<0.05$

d P-value $<0.001$ 
Table A1a (continued). Indicator variable baseline (intercept) and annual change (slope) estimates by campsite. Variable baseline of predicted 2008 value (Yearly Change). For all variables, a negative yearly change indicates improvement, 0 is no change, positive yearly change indicates degradation.

\begin{tabular}{|c|c|c|c|c|c|c|c|c|c|c|}
\hline Site & $\begin{array}{r}\text { Tag } \\
\text { Number }\end{array}$ & Area $\left(m^{2}\right)$ & $\begin{array}{r}\text { Condition } \\
\text { Class }\end{array}$ & $\begin{array}{r}\text { Vegetation } \\
\text { Loss (\%) }\end{array}$ & $\begin{array}{r}\text { Tent } \\
\text { Rocks }\end{array}$ & Trails & $\begin{array}{r}\text { Ghost Tree } \\
\text { Damage }\end{array}$ & Trash & $\begin{array}{c}\text { Human } \\
\text { Waste }\end{array}$ & $\begin{array}{r}\text { Latitude }\left({ }^{\circ} \mathrm{N}\right) \\
\text { Longitude }\left({ }^{\circ} \mathrm{W}\right)\end{array}$ \\
\hline \multirow{4}{*}{$\begin{array}{l}\text { Verdant } \\
\text { (continued) }\end{array}$} & $105^{b}$ & 64.59 & 7.50 & NA & 40.00 & 3.50 & 1.00 & 0.00 & 1.50 & 59.6984 \\
\hline & & $(-12.92)^{a}$ & $(-1.50)^{a}$ & & $(-8.00)^{a}$ & $(-0.50)^{a}$ & $(0.00)^{a}$ & $(0.00)^{a}$ & $(-0.25)^{a}$ & 149.7334 \\
\hline & 106 & -13.84 & 3.50 & 27.98 & -8.25 & 2.50 & 1.00 & 0.00 & 0.00 & 59.6981 \\
\hline & & $(9.20)^{a}$ & $(-0.50)^{a}$ & $(-10.32)^{a}$ & $(3.75)^{a}$ & $(-0.50)^{a}$ & $(0.00)^{a}$ & $(0.00)^{a}$ & $(0.00)^{a}$ & 149.7343 \\
\hline
\end{tabular}

\footnotetext{
a Sample size of 2. P-value is not attainable.

${ }^{\mathrm{b}}$ Recovered campsite

c $P$-value $<0.05$

d $P$-value $<0.001$
} 
Table A1b. Indicator variable baseline (intercept) and annual change (slope) estimates by campsite. Variable baseline of predicted 2008 value (Yearly Change). For all variables, a negative yearly change indicates improvement, 0 is no change, positive yearly change indicates degradation.

\begin{tabular}{|c|c|c|c|c|c|c|c|}
\hline Site & $\begin{array}{r}\text { Tag } \\
\text { Number }\end{array}$ & $\begin{array}{l}\text { Mineral } \\
\text { Soil (\%) }\end{array}$ & $\begin{array}{r}\text { Fire } \\
\text { Rings }\end{array}$ & $\begin{array}{r}\text { Tree } \\
\text { Stumps }\end{array}$ & $\begin{array}{r}\text { Tree } \\
\text { Damage }\end{array}$ & $\begin{array}{r}\text { Root } \\
\text { Exposure }\end{array}$ & $\begin{array}{r}\text { Latitude }\left({ }^{\circ} \mathrm{N}\right) \\
\text { Longitude }\left({ }^{\circ} \mathrm{W}\right)\end{array}$ \\
\hline \multirow[t]{14}{*}{ Abra } & \multirow[t]{2}{*}{67} & 38.00 & 0.00 & 0.00 & 2.67 & 2.67 & 59.8945 \\
\hline & & $(0.00)^{\mathrm{a}}$ & $(0.00)^{a}$ & $(0.00)^{a}$ & $(-0.33)^{a}$ & $(-0.33)^{a}$ & 149.6438 \\
\hline & \multirow[t]{2}{*}{68} & 85.00 & 0.00 & 0.00 & 2.67 & 1.00 & 59.8944 \\
\hline & & $(0.00)^{\mathrm{a}}$ & $(0.00)^{a}$ & $(0.00)^{a}$ & $(-0.33)^{a}$ & $(0.00)^{a}$ & 149.6440 \\
\hline & \multirow[t]{2}{*}{69} & 98.00 & 0.00 & 0.00 & 1.00 & 1.00 & 59.8942 \\
\hline & & $(0.00)^{a}$ & $(0.00)^{a}$ & $(0.00)^{a}$ & $(0.00)^{\mathrm{a}}$ & $(0.00)^{a}$ & 149.6447 \\
\hline & \multirow[t]{2}{*}{74} & 29.43 & 0.00 & 0.00 & 1.00 & 1.57 & 59.8943 \\
\hline & & $(9.82)$ & $(0.00)^{a}$ & $(0.00)^{a}$ & $(0.00)^{a}$ & $(-0.07)^{\mathrm{a}}$ & 149.6423 \\
\hline & \multirow[t]{2}{*}{75} & 120.86 & 0.00 & 0.00 & 1.43 & 0.71 & 59.8944 \\
\hline & & $(-11.61)^{c}$ & $(0.00)^{a}$ & $(0.00)^{\mathrm{a}}$ & $(0.07)^{\mathrm{a}}$ & $(0.29)^{a}$ & 149.6443 \\
\hline & \multirow[t]{2}{*}{90} & 71.75 & 0.00 & 0.00 & 1.00 & 3.50 & 59.8944 \\
\hline & & $(-11.25)^{\mathrm{a}}$ & $(0.00)^{a}$ & $(0.00)^{a}$ & $(0.00)^{\mathrm{a}}$ & $(-0.50)^{\mathrm{a}}$ & 149.6425 \\
\hline & \multirow[t]{2}{*}{91} & -52.00 & 0.00 & 0.00 & 1.00 & 1.00 & 59.8943 \\
\hline & & $(30.00)^{a}$ & $(0.00)^{a}$ & $(0.00)^{a}$ & $(0.00)^{a}$ & $(0.00)^{a}$ & 149.6419 \\
\hline \multirow{12}{*}{ Bear Cove } & \multirow[t]{2}{*}{18} & 120.08 & 0.00 & 5.42 & 1.00 & 1.00 & 59.7907 \\
\hline & & $(-20.75)$ & $(0.00)^{a}$ & $(-1.25)$ & $(0.00)^{a}$ & $(0.00)^{a}$ & 149.6167 \\
\hline & \multirow[t]{2}{*}{38} & 85.50 & 0.33 & -0.42 & 1.00 & 1.00 & 59.7896 \\
\hline & & $(0.00)$ & $(0.00)$ & $(0.25)$ & $(0.00)^{a}$ & $(0.00)^{\mathrm{a}}$ & 149.6167 \\
\hline & \multirow[t]{2}{*}{39} & 84.46 & 0.00 & -0.42 & 1.00 & 1.00 & 59.7897 \\
\hline & & $(3.12)$ & $(0.00)^{\mathrm{a}}$ & $(0.25)$ & $(0.00)^{a}$ & $(0.00)^{a}$ & 149.6167 \\
\hline & \multirow[t]{2}{*}{40} & 48.42 & 0.00 & -0.42 & 1.00 & 1.67 & 59.7898 \\
\hline & & $(8.75)$ & $(0.00)^{a}$ & $(0.25)$ & $(0.00)^{\mathrm{a}}$ & $(0.00)^{a}$ & 149.6167 \\
\hline & \multirow[t]{2}{*}{41} & 52.79 & 0.33 & 0.00 & 1.00 & 1.00 & 59.7903 \\
\hline & & $(-8.88)$ & $(0.00)$ & $(0.00)^{a}$ & $(0.00)^{a}$ & $(0.00)^{a}$ & 149.6166 \\
\hline & \multirow[t]{2}{*}{$42^{\mathrm{b}}$} & 2.5 & 0.00 & 0.00 & 1.00 & 1.00 & 59.7905 \\
\hline & & $(0.00)$ & $(0.00)^{a}$ & $(0.00)^{a}$ & $(0.00)^{a}$ & $(0.00)^{a}$ & 149.6161 \\
\hline \multirow[t]{4}{*}{ Bulldog } & \multirow[t]{2}{*}{76} & 92.64 & 0.00 & 0.00 & 2.86 & 1.00 & 59.8913 \\
\hline & & $(-0.89)$ & $(0.00)^{\mathrm{a}}$ & $(0.00)^{\mathrm{a}}$ & $(-0.36)^{a}$ & $(0.00)^{\mathrm{a}}$ & 149.5580 \\
\hline & \multirow[t]{2}{*}{$77^{\mathrm{b}}$} & 63.00 & -0.86 & 0.00 & 2.86 & 2.86 & 59.8914 \\
\hline & & $(0.00)^{\mathrm{a}}$ & $(0.36)$ & $(0.00)^{\mathrm{a}}$ & $(-0.36)^{a}$ & $(-0.36)^{a}$ & 149.5577 \\
\hline
\end{tabular}

\footnotetext{
a Sample size of 2. P-value is not attainable.

${ }^{\mathrm{b}}$ Recovered campsite

${ }^{\mathrm{c}} \mathrm{P}$-value $<0.05$
} 
Table A1b (continued). Indicator variable baseline (intercept) and annual change (slope) estimates by campsite. Variable baseline of predicted 2008 value (Yearly Change). For all variables, a negative yearly change indicates improvement, 0 is no change, positive yearly change indicates degradation.

\begin{tabular}{|c|c|c|c|c|c|c|c|}
\hline Site & $\begin{array}{r}\text { Tag } \\
\text { Number }\end{array}$ & $\begin{array}{l}\text { Mineral } \\
\text { Soil (\%) }\end{array}$ & $\begin{array}{r}\text { Fire } \\
\text { Rings }\end{array}$ & $\begin{array}{r}\text { Tree } \\
\text { Stumps }\end{array}$ & $\begin{array}{r}\text { Tree } \\
\text { Damage }\end{array}$ & $\begin{array}{r}\text { Root } \\
\text { Exposure }\end{array}$ & $\begin{array}{r}\text { Latitude }\left({ }^{\circ} \mathrm{N}\right) \\
\text { Longitude }\left({ }^{\circ} \mathrm{W}\right)\end{array}$ \\
\hline \multirow[t]{3}{*}{$\begin{array}{l}\text { Bulldog } \\
\text { (continued) }\end{array}$} & 78 & $\begin{array}{r}2.50 \\
(0.00)^{\mathrm{a}}\end{array}$ & $\begin{array}{r}1.00 \\
(0.00)^{\mathrm{a}}\end{array}$ & $\begin{array}{r}0.00 \\
(0.00)^{\mathrm{a}}\end{array}$ & $\begin{array}{r}1.00 \\
(0.00)^{\mathrm{a}}\end{array}$ & $\begin{array}{r}1.00 \\
(0.00)^{\mathrm{a}}\end{array}$ & $\begin{array}{r}59.8921 \\
149.5632\end{array}$ \\
\hline & $80^{b}$ & $\begin{array}{r}145.50 \\
(-25.00)\end{array}$ & $\begin{array}{r}1.29 \\
(-0.29)\end{array}$ & $\begin{array}{r}0.00 \\
(0.00)^{\mathrm{a}}\end{array}$ & $\begin{array}{r}2.86 \\
(-0.36)^{\mathrm{a}}\end{array}$ & $\begin{array}{r}2.29 \\
(-0.29)^{a}\end{array}$ & $\begin{array}{r}59.8912 \\
149.5572\end{array}$ \\
\hline & 81 & $\begin{array}{l}85.50 \\
(0.00)\end{array}$ & $\begin{array}{r}1.29 \\
(-0.29)\end{array}$ & $\begin{array}{r}0.00 \\
(0.00)^{\mathrm{a}}\end{array}$ & $\begin{array}{r}2.71 \\
(-0.21)^{a}\end{array}$ & $\begin{array}{r}2.86 \\
(-0.36)^{a}\end{array}$ & $\begin{array}{r}59.8913 \\
149.5572\end{array}$ \\
\hline \multirow[t]{6}{*}{ Holgate North } & $31^{b}$ & $\begin{array}{r}38.00 \\
(0.00)^{\mathrm{a}}\end{array}$ & $\begin{array}{r}0.00 \\
(0.00)^{\mathrm{a}}\end{array}$ & $\begin{array}{r}0.00 \\
(0.00)^{\mathrm{a}}\end{array}$ & $\begin{array}{r}1.33 \\
(0.00)^{\mathrm{a}}\end{array}$ & $\begin{array}{r}1.33 \\
(0.00)^{\mathrm{a}}\end{array}$ & $\begin{array}{r}59.8456 \\
149.7887\end{array}$ \\
\hline & 32 & $\begin{array}{r}94.88 \\
(-5.63)\end{array}$ & $\begin{array}{r}0.00 \\
(0.00)^{\mathrm{a}}\end{array}$ & $\begin{array}{r}0.00 \\
(0.00)^{\mathrm{a}}\end{array}$ & $\begin{array}{r}1.33 \\
(0.00)^{\mathrm{a}}\end{array}$ & $\begin{array}{r}1.33 \\
(0.00)^{\mathrm{a}}\end{array}$ & $\begin{array}{r}59.8456 \\
149.7889\end{array}$ \\
\hline & 33 & $\begin{array}{r}87.38 \\
(-5.63)\end{array}$ & $\begin{array}{r}0.00 \\
(0.00)^{\mathrm{a}}\end{array}$ & $\begin{array}{r}0.00 \\
(0.00)^{\mathrm{a}}\end{array}$ & $\begin{array}{r}1.33 \\
(0.00)^{\mathrm{a}}\end{array}$ & $\begin{array}{r}1.33 \\
(0.00)^{\mathrm{a}}\end{array}$ & $\begin{array}{r}59.8455 \\
149.7890\end{array}$ \\
\hline & $34^{b}$ & $\begin{array}{r}51.75 \\
(11.25)^{\mathrm{a}}\end{array}$ & $\begin{array}{r}0.00 \\
(0.00)^{\mathrm{a}}\end{array}$ & $\begin{array}{r}0.00 \\
(0.00)^{\mathrm{a}}\end{array}$ & $\begin{array}{r}1.00 \\
(0.00)^{\mathrm{a}}\end{array}$ & $\begin{array}{r}0.50 \\
(0.50)^{\mathrm{a}}\end{array}$ & $\begin{array}{r}59.8453 \\
149.7888\end{array}$ \\
\hline & $35^{b}$ & $\begin{array}{r}75.50 \\
(-12.50)^{\mathrm{a}}\end{array}$ & $\begin{array}{r}0.00 \\
(0.00)^{\mathrm{a}}\end{array}$ & $\begin{array}{r}0.00 \\
(0.00)^{\mathrm{a}}\end{array}$ & $\begin{array}{r}1.33 \\
(0.00)^{\mathrm{a}}\end{array}$ & $\begin{array}{r}1.33 \\
(0.00)^{\mathrm{a}}\end{array}$ & $\begin{array}{r}59.8454 \\
149.7888\end{array}$ \\
\hline & 36 & $\begin{array}{r}75.50 \\
(-12.50)^{\mathrm{a}}\end{array}$ & $\begin{array}{r}0.00 \\
(0.00)^{\mathrm{a}}\end{array}$ & $\begin{array}{r}0.00 \\
(0.00)^{\mathrm{a}}\end{array}$ & $\begin{array}{r}0.50 \\
(0.50)^{\mathrm{a}}\end{array}$ & $\begin{array}{r}1.00 \\
(0.00)^{\mathrm{a}}\end{array}$ & $\begin{array}{r}59.8457 \\
149.7890\end{array}$ \\
\hline \multirow[t]{3}{*}{ Holgate South } & 2 & $\begin{array}{r}63.00 \\
(0.00)^{\mathrm{a}}\end{array}$ & $\begin{array}{r}0.00 \\
(0.00)^{\mathrm{a}}\end{array}$ & $\begin{array}{r}0.00 \\
(0.00)^{\mathrm{a}}\end{array}$ & $\begin{array}{r}1.00 \\
(0.00)^{\mathrm{a}}\end{array}$ & $\begin{array}{r}1.00 \\
(0.00)^{\mathrm{a}}\end{array}$ & $\begin{array}{r}59.8318 \\
149.7688\end{array}$ \\
\hline & $64^{b}$ & NA & $\begin{array}{r}0.00 \\
(0.00)^{\mathrm{a}}\end{array}$ & $\begin{array}{r}0.00 \\
(0.00)^{\mathrm{a}}\end{array}$ & $\begin{array}{r}2.67 \\
(-0.33)^{\mathrm{a}}\end{array}$ & $\begin{array}{r}2.67 \\
(-0.33)^{\mathrm{a}}\end{array}$ & $\begin{array}{r}59.8365 \\
149.7717\end{array}$ \\
\hline & $72^{b}$ & $\begin{array}{r}156.64 \\
(-29.64)\end{array}$ & $\begin{array}{r}0.00 \\
(0.00)^{\mathrm{a}}\end{array}$ & $\begin{array}{r}0.00 \\
(0.00)^{\mathrm{a}}\end{array}$ & $\begin{array}{r}1.00 \\
(0.00)^{\mathrm{a}}\end{array}$ & $\begin{array}{r}1.00 \\
(0.00)^{\mathrm{a}}\end{array}$ & $\begin{array}{r}59.8380 \\
149.7740\end{array}$ \\
\hline \multirow[t]{4}{*}{ McMullen } & 84 & $\begin{array}{r}98.00 \\
(0.00)^{\mathrm{a}}\end{array}$ & $\begin{array}{r}0.00 \\
(0.00)^{\mathrm{a}}\end{array}$ & $\begin{array}{r}0.00 \\
(0.00)^{\mathrm{a}}\end{array}$ & $\begin{array}{r}1.33 \\
(0.00)^{\mathrm{a}}\end{array}$ & $\begin{array}{r}1.00 \\
(0.00)^{\mathrm{a}}\end{array}$ & $\begin{array}{r}59.7634 \\
149.7685\end{array}$ \\
\hline & 85 & $\begin{array}{r}98.00 \\
(0.00)^{\mathrm{a}}\end{array}$ & $\begin{array}{l}-0.42 \\
(0.25)\end{array}$ & $\begin{array}{r}0.00 \\
(0.00)^{\mathrm{a}}\end{array}$ & $\begin{array}{r}1.00 \\
(0.00)^{\mathrm{a}}\end{array}$ & $\begin{array}{r}1.00 \\
(0.00)^{\mathrm{a}}\end{array}$ & $\begin{array}{r}59.7635 \\
149.7684\end{array}$ \\
\hline & 104 & $\begin{array}{r}98.00 \\
(0.00)^{\mathrm{a}}\end{array}$ & $\begin{array}{r}0.00 \\
(0.00)^{\mathrm{a}}\end{array}$ & $\begin{array}{r}0.00 \\
(0.00)^{\mathrm{a}}\end{array}$ & $\begin{array}{r}1.00 \\
(0.00)^{\mathrm{a}}\end{array}$ & $\begin{array}{r}1.00 \\
(0.00)^{\mathrm{a}}\end{array}$ & $\begin{array}{r}59.7633 \\
149.7686\end{array}$ \\
\hline & 114 & NA & $\begin{array}{r}0.75 \\
(0.25)^{\mathrm{a}}\end{array}$ & $\begin{array}{r}0.00 \\
(0.00)^{\mathrm{a}}\end{array}$ & $\begin{array}{r}1.00 \\
(0.00)^{\mathrm{a}}\end{array}$ & $\begin{array}{r}1.00 \\
(0.00)^{\mathrm{a}}\end{array}$ & $\begin{array}{r}59.7627 \\
149.7684\end{array}$ \\
\hline
\end{tabular}

\footnotetext{
a Sample size of 2. P-value is not attainable.

${ }^{\mathrm{b}}$ Recovered campsite

c P-value $<0.05$
} 
Table A1b (continued). Indicator variable baseline (intercept) and annual change (slope) estimates by campsite. Variable baseline of predicted 2008 value (Yearly Change). For all variables, a negative yearly change indicates improvement, 0 is no change, positive yearly change indicates degradation.

\begin{tabular}{|c|c|c|c|c|c|c|c|}
\hline Site & $\begin{array}{r}\text { Tag } \\
\text { Number }\end{array}$ & $\begin{array}{l}\text { Mineral } \\
\text { Soil (\%) }\end{array}$ & $\begin{array}{r}\text { Fire } \\
\text { Rings }\end{array}$ & $\begin{array}{r}\text { Tree } \\
\text { Stumps }\end{array}$ & $\begin{array}{r}\text { Tree } \\
\text { Damage }\end{array}$ & $\begin{array}{r}\text { Root } \\
\text { Exposure }\end{array}$ & $\begin{array}{r}\text { Latitude }\left({ }^{\circ} \mathrm{N}\right) \\
\text { Longitude }\left({ }^{\circ} \mathrm{W}\right)\end{array}$ \\
\hline \multirow[t]{11}{*}{ Northeastern } & 50 & $\begin{array}{r}57.29 \\
(-8.04)^{\mathrm{a}}\end{array}$ & $\begin{array}{r}-0.86 \\
(0.36)\end{array}$ & $\begin{array}{r}-0.86 \\
(0.36)\end{array}$ & $\begin{array}{r}2.71 \\
(-0.21)^{\mathrm{a}}\end{array}$ & $\begin{array}{r}2.86 \\
(-0.36)^{a}\end{array}$ & $\begin{array}{r}59.8016 \\
150.0138\end{array}$ \\
\hline & 51 & $\begin{array}{r}2.50 \\
(0.00)\end{array}$ & $\begin{array}{l}-0.86 \\
(0.36)\end{array}$ & $\begin{array}{r}0.00 \\
(0.00)^{\mathrm{a}}\end{array}$ & $\begin{array}{r}2.71 \\
(-0.21)^{\mathrm{a}}\end{array}$ & $\begin{array}{r}2.86 \\
(-0.36)^{a}\end{array}$ & $\begin{array}{r}59.8018 \\
150.0138\end{array}$ \\
\hline & 52 & $\begin{array}{l}15.50 \\
(0.00)\end{array}$ & $\begin{array}{r}0.00 \\
(0.00)^{\mathrm{a}}\end{array}$ & $\begin{array}{r}0.00 \\
(0.00)^{\mathrm{a}}\end{array}$ & $\begin{array}{r}2.86 \\
(-0.36)^{a}\end{array}$ & $\begin{array}{r}2.86 \\
(-0.36)^{a}\end{array}$ & $\begin{array}{r}59.8015 \\
150.0137\end{array}$ \\
\hline & 53 & $\begin{array}{r}2.50 \\
(0.00)\end{array}$ & $\begin{array}{r}0.00 \\
(0.00)^{\mathrm{a}}\end{array}$ & $\begin{array}{r}0.00 \\
(0.00)^{\mathrm{a}}\end{array}$ & $\begin{array}{r}2.86 \\
(-0.36)^{\mathrm{a}}\end{array}$ & $\begin{array}{r}2.86 \\
(-0.36)^{\mathrm{a}}\end{array}$ & $\begin{array}{r}59.8015 \\
150.0135\end{array}$ \\
\hline & 54 & $\begin{array}{l}63.00 \\
(0.00)\end{array}$ & $\begin{array}{r}0.00 \\
(0.00)^{\mathrm{a}}\end{array}$ & $\begin{array}{r}0.00 \\
(0.00)^{\mathrm{a}}\end{array}$ & $\begin{array}{r}0.36 \\
(0.36)^{\mathrm{a}}\end{array}$ & $\begin{array}{r}1.00 \\
(0.00)^{\mathrm{a}}\end{array}$ & $\begin{array}{r}59.8007 \\
150.0126\end{array}$ \\
\hline & 55 & $\begin{array}{r}79.00 \\
(-4.75)\end{array}$ & $\begin{array}{r}0.00 \\
(0.00)^{\mathrm{a}}\end{array}$ & $\begin{array}{r}0.00 \\
(0.00)^{\mathrm{a}}\end{array}$ & $\begin{array}{r}1.00 \\
(0.00)^{\mathrm{a}}\end{array}$ & $\begin{array}{r}1.00 \\
(0.00)^{\mathrm{a}}\end{array}$ & $\begin{array}{r}59.8009 \\
150.0126\end{array}$ \\
\hline & 58 & $\begin{array}{l}101.57 \\
(-3.57)\end{array}$ & $\begin{array}{r}2.57 \\
(-0.57)\end{array}$ & $\begin{array}{r}0.00 \\
(0.00)^{\mathrm{a}}\end{array}$ & $\begin{array}{r}1.00 \\
(0.00)^{\mathrm{a}}\end{array}$ & $\begin{array}{r}1.00 \\
(0.00)^{\mathrm{a}}\end{array}$ & $\begin{array}{r}59.8004 \\
150.0123\end{array}$ \\
\hline & 59 & $\begin{array}{r}117.17 \\
(-15.83)^{\mathrm{a}}\end{array}$ & $\begin{array}{r}0.00 \\
(0.00)^{\mathrm{a}}\end{array}$ & $\begin{array}{r}0.00 \\
(0.00)^{\mathrm{a}}\end{array}$ & $\begin{array}{r}1.00 \\
(0.00)^{\mathrm{a}}\end{array}$ & $\begin{array}{r}1.00 \\
(0.00)^{\mathrm{a}}\end{array}$ & $\begin{array}{r}59.8006 \\
150.0125\end{array}$ \\
\hline & 60 & $\begin{array}{l}103.50 \\
(-6.75)\end{array}$ & $\begin{array}{r}0.00 \\
(0.00)^{\mathrm{a}}\end{array}$ & $\begin{array}{r}0.00 \\
(0.00)^{\mathrm{a}}\end{array}$ & $\begin{array}{r}2.20 \\
(-0.20)^{\mathrm{a}}\end{array}$ & $\begin{array}{r}2.80 \\
(-0.30)^{a}\end{array}$ & $\begin{array}{r}59.8005 \\
150.0128\end{array}$ \\
\hline & 88 & $\begin{array}{r}66.75 \\
(6.25)^{\mathrm{a}}\end{array}$ & $\begin{array}{r}0.00 \\
(0.00)^{\mathrm{a}}\end{array}$ & $\begin{array}{r}0.00 \\
(0.00)^{\mathrm{a}}\end{array}$ & $\begin{array}{r}3.50 \\
(-0.50)^{\mathrm{a}}\end{array}$ & $\begin{array}{r}3.50 \\
(-0.50)^{\mathrm{a}}\end{array}$ & $\begin{array}{r}59.8004 \\
150.0125\end{array}$ \\
\hline & 89 & $\begin{array}{l}98.00 \\
(0.00)\end{array}$ & $\begin{array}{r}0.00 \\
(0.00)^{\mathrm{a}}\end{array}$ & $\begin{array}{r}0.00 \\
(0.00)^{\mathrm{a}}\end{array}$ & $\begin{array}{r}1.00 \\
(0.00)^{\mathrm{a}}\end{array}$ & $\begin{array}{r}1.00 \\
(0.00)^{\mathrm{a}}\end{array}$ & $\begin{array}{r}59.8004 \\
150.0124\end{array}$ \\
\hline \multirow[t]{4}{*}{ NW Landing } & 46 & $\begin{array}{r}18.00 \\
(22.50)^{\mathrm{a}}\end{array}$ & $\begin{array}{r}3.00 \\
(-1.00)^{\mathrm{a}}\end{array}$ & $\begin{array}{r}12.00 \\
(-4.00)^{\mathrm{a}}\end{array}$ & $\begin{array}{r}1.00 \\
(0.00)^{\mathrm{a}}\end{array}$ & $\begin{array}{r}1.00 \\
(0.00)^{\mathrm{a}}\end{array}$ & $\begin{array}{r}59.7563 \\
149.8944\end{array}$ \\
\hline & 47 & $\begin{array}{r}-23.50 \\
(13.00)^{\mathrm{a}}\end{array}$ & $\begin{array}{r}0.00 \\
(0.00)^{\mathrm{a}}\end{array}$ & $\begin{array}{r}3.00 \\
(-1.00)^{a}\end{array}$ & $\begin{array}{r}4.00 \\
(-1.00)^{\mathrm{a}}\end{array}$ & $\begin{array}{r}4.00 \\
(-1.00)^{a}\end{array}$ & $\begin{array}{r}59.7564 \\
149.8945\end{array}$ \\
\hline & 48 & $\begin{array}{r}2.50 \\
(0.00)^{\mathrm{a}}\end{array}$ & $\begin{array}{r}0.00 \\
(0.00)^{\mathrm{a}}\end{array}$ & $\begin{array}{r}0.00 \\
(0.00)^{\mathrm{a}}\end{array}$ & $\begin{array}{r}1.00 \\
(0.00)^{\mathrm{a}}\end{array}$ & $\begin{array}{r}1.00 \\
(0.00)^{\mathrm{a}}\end{array}$ & $\begin{array}{r}59.7565 \\
149.8945\end{array}$ \\
\hline & 49 & $\begin{array}{r}2.50 \\
(0.00)^{\mathrm{a}}\end{array}$ & $\begin{array}{r}0.00 \\
(0.00)^{\mathrm{a}}\end{array}$ & $\begin{array}{r}0.00 \\
(0.00)^{\mathrm{a}}\end{array}$ & $\begin{array}{r}1.00 \\
(0.00)^{\mathrm{a}}\end{array}$ & $\begin{array}{r}1.00 \\
(0.00)^{a}\end{array}$ & $\begin{array}{r}59.7566 \\
149.8945\end{array}$ \\
\hline
\end{tabular}

a Sample size of 2. P-value is not attainable.

${ }^{b}$ Recovered campsite

${ }^{c} \mathrm{P}$-value $<0.05$ 
Table A1b (continued). Indicator variable baseline (intercept) and annual change (slope) estimates by campsite. Variable baseline of predicted 2008 value (Yearly Change). For all variables, a negative yearly change indicates improvement, 0 is no change, positive yearly change indicates degradation.

\begin{tabular}{|c|c|c|c|c|c|c|c|}
\hline Site & $\begin{array}{r}\text { Tag } \\
\text { Number }\end{array}$ & $\begin{array}{l}\text { Mineral } \\
\text { Soil (\%) }\end{array}$ & $\begin{array}{r}\text { Fire } \\
\text { Rings }\end{array}$ & $\begin{array}{r}\text { Tree } \\
\text { Stumps }\end{array}$ & $\begin{array}{r}\text { Tree } \\
\text { Damage }\end{array}$ & $\begin{array}{r}\text { Root } \\
\text { Exposure }\end{array}$ & $\begin{array}{r}\text { Latitude }\left({ }^{\circ} \mathrm{N}\right) \\
\text { Longitude }\left({ }^{\circ} \mathrm{W}\right)\end{array}$ \\
\hline \multirow[t]{13}{*}{ Pedersen } & 5 & $\begin{array}{r}-41.93 \\
(20.68)\end{array}$ & $\begin{array}{r}1.00 \\
(0.00)^{\mathrm{a}}\end{array}$ & $\begin{array}{r}1.57 \\
(-0.07)\end{array}$ & $\begin{array}{r}2.29 \\
(-0.29)^{a}\end{array}$ & $\begin{array}{r}1.00 \\
(0.00)^{\mathrm{a}}\end{array}$ & $\begin{array}{r}59.8796 \\
149.7369\end{array}$ \\
\hline & 6 & $\begin{array}{r}2.50 \\
(0.00)\end{array}$ & $\begin{array}{r}1.29 \\
(-0.29)\end{array}$ & $\begin{array}{r}0.00 \\
(0.00)^{\mathrm{a}}\end{array}$ & $\begin{array}{r}1.00 \\
(0.00)^{\mathrm{a}}\end{array}$ & $\begin{array}{r}1.57 \\
(-0.07)^{\mathrm{a}}\end{array}$ & $\begin{array}{r}59.8797 \\
149.7370\end{array}$ \\
\hline & 7 & $\begin{array}{r}-88.25 \\
(30.25)^{\mathrm{a}}\end{array}$ & $\begin{array}{r}-1.50 \\
(0.50)^{\mathrm{a}}\end{array}$ & $\begin{array}{r}-1.50 \\
(0.50)^{\mathrm{a}}\end{array}$ & $\begin{array}{r}3.50 \\
(-0.50)^{\mathrm{a}}\end{array}$ & $\begin{array}{r}1.00 \\
(0.00)^{a}\end{array}$ & $\begin{array}{r}59.8798 \\
149.7367\end{array}$ \\
\hline & 8 & $\begin{array}{r}2.50 \\
(0.00)^{\mathrm{a}}\end{array}$ & $\begin{array}{r}1.00 \\
(0.00)^{\mathrm{a}}\end{array}$ & $\begin{array}{r}0.00 \\
(0.00)^{\mathrm{a}}\end{array}$ & $\begin{array}{r}1.00 \\
(0.00)^{\mathrm{a}}\end{array}$ & $\begin{array}{r}-0.50 \\
(0.50)^{\mathrm{a}}\end{array}$ & $\begin{array}{r}59.8797 \\
149.7366\end{array}$ \\
\hline & 9 & $\begin{array}{r}-50.75 \\
(17.75)^{\mathrm{a}}\end{array}$ & $\begin{array}{r}-1.50 \\
(0.50)^{\mathrm{a}}\end{array}$ & $\begin{array}{r}0.00 \\
(0.00)^{\mathrm{a}}\end{array}$ & $\begin{array}{r}3.50 \\
(-0.50)^{\mathrm{a}}\end{array}$ & $\begin{array}{r}1.00 \\
(0.00)^{\mathrm{a}}\end{array}$ & $\begin{array}{r}59.8797 \\
149.7366\end{array}$ \\
\hline & $11^{b}$ & $\begin{array}{r}2.50 \\
(0.00)^{\mathrm{a}}\end{array}$ & $\begin{array}{r}0.00 \\
(0.00)^{\mathrm{a}}\end{array}$ & $\begin{array}{r}0.00 \\
(0.00)^{\mathrm{a}}\end{array}$ & $\begin{array}{r}1.00 \\
(0.00)^{\mathrm{a}}\end{array}$ & $\begin{array}{r}-0.50 \\
(0.50)^{\mathrm{a}}\end{array}$ & $\begin{array}{r}59.8796 \\
149.7373\end{array}$ \\
\hline & 12 & $\begin{array}{r}2.50 \\
(0.00)\end{array}$ & $\begin{array}{r}1.29 \\
(-0.29)\end{array}$ & $\begin{array}{r}-0.86 \\
(0.36)\end{array}$ & $\begin{array}{r}1.00 \\
(0.00)^{\mathrm{a}}\end{array}$ & $\begin{array}{r}2.29 \\
(-0.29)^{a}\end{array}$ & $\begin{array}{r}59.8798 \\
149.7370\end{array}$ \\
\hline & 13 & $\begin{array}{r}-6.17 \\
(4.33)^{\mathrm{a}}\end{array}$ & $\begin{array}{r}0.00 \\
(0.00)^{\mathrm{a}}\end{array}$ & $\begin{array}{r}-1.33 \\
(0.67)^{\mathrm{a}}\end{array}$ & $\begin{array}{r}1.00 \\
(0.00)^{\mathrm{a}}\end{array}$ & $\begin{array}{r}1.00 \\
(0.00)^{\mathrm{a}}\end{array}$ & $\begin{array}{r}59.8797 \\
149.7369\end{array}$ \\
\hline & 14 & $\begin{array}{r}-27.93 \\
(12.68)\end{array}$ & $\begin{array}{r}0.00 \\
(0.00)^{\mathrm{a}}\end{array}$ & $\begin{array}{r}-0.86 \\
(0.36)\end{array}$ & $\begin{array}{r}1.00 \\
(0.00)^{\mathrm{a}}\end{array}$ & $\begin{array}{r}2.29 \\
(-0.29)^{a}\end{array}$ & $\begin{array}{r}59.8799 \\
149.7367\end{array}$ \\
\hline & 16 & $\begin{array}{l}43.71 \\
(8.04)\end{array}$ & $\begin{array}{l}-0.29 \\
(0.29)\end{array}$ & $\begin{array}{r}0.00 \\
(0.00)^{\mathrm{a}}\end{array}$ & $\begin{array}{r}1.00 \\
(0.00)^{\mathrm{a}}\end{array}$ & $\begin{array}{r}1.00 \\
(0.00)^{\mathrm{a}}\end{array}$ & $\begin{array}{r}59.8795 \\
149.7371\end{array}$ \\
\hline & 79 & $\begin{array}{r}2.50 \\
(0.00)\end{array}$ & $\begin{array}{r}0.00 \\
(0.00)^{\mathrm{a}}\end{array}$ & $\begin{array}{r}0.43 \\
(0.71)\end{array}$ & $\begin{array}{r}2.67 \\
(-0.33)^{a}\end{array}$ & $\begin{array}{r}1.43 \\
(0.07)^{\mathrm{a}}\end{array}$ & $\begin{array}{r}59.8799 \\
149.7364\end{array}$ \\
\hline & 92 & $\begin{array}{r}-50.75 \\
(17.75)^{\mathrm{a}}\end{array}$ & $\begin{array}{r}0.00 \\
(0.00)^{\mathrm{a}}\end{array}$ & $\begin{array}{r}3.50 \\
(-0.50)^{\mathrm{a}}\end{array}$ & $\begin{array}{r}1.00 \\
(0.00)^{\mathrm{a}}\end{array}$ & $\begin{array}{r}-0.50 \\
(0.50)^{\mathrm{a}}\end{array}$ & $\begin{array}{r}59.8795 \\
149.7368\end{array}$ \\
\hline & 93 & $\begin{array}{r}2.50 \\
(0.00)^{\mathrm{a}}\end{array}$ & $\begin{array}{r}0.00 \\
(0.00)^{\mathrm{a}}\end{array}$ & $\begin{array}{r}3.00 \\
(0.00)^{\mathrm{a}}\end{array}$ & $\begin{array}{r}1.00 \\
(0.00)^{\mathrm{a}}\end{array}$ & $\begin{array}{r}1.00 \\
(0.00)^{\mathrm{a}}\end{array}$ & $\begin{array}{r}59.8795 \\
149.7367\end{array}$ \\
\hline \multirow[t]{2}{*}{ Pocket Cove } & 65 & $\begin{array}{l}108.71 \\
(-4.46)\end{array}$ & $\begin{array}{r}0.00 \\
(0.00)^{\mathrm{a}}\end{array}$ & $\begin{array}{r}0.00 \\
(0.00)^{\mathrm{a}}\end{array}$ & $\begin{array}{r}1.00 \\
(0.00)^{\mathrm{a}}\end{array}$ & $\begin{array}{r}2.29 \\
(-0.29)^{a}\end{array}$ & $\begin{array}{r}59.8533 \\
149.6577\end{array}$ \\
\hline & 73 & $\begin{array}{r}77.17 \\
(4.17)^{\mathrm{a}}\end{array}$ & $\begin{array}{r}1.00 \\
(0.00)^{\mathrm{a}}\end{array}$ & $\begin{array}{r}-0.67 \\
(0.33)^{\mathrm{a}}\end{array}$ & $\begin{array}{r}0.33 \\
(0.33)^{\mathrm{a}}\end{array}$ & $\begin{array}{r}0.33 \\
(0.33)^{\mathrm{a}}\end{array}$ & $\begin{array}{r}59.8535 \\
149.6577\end{array}$ \\
\hline
\end{tabular}

a Sample size of 2. P-value is not attainable.

${ }^{b}$ Recovered campsite

${ }^{c} \mathrm{P}$-value $<0.05$ 
Table A1b (continued). Indicator variable baseline (intercept) and annual change (slope) estimates by campsite. Variable baseline of predicted 2008 value (Yearly Change). For all variables, a negative yearly change indicates improvement, 0 is no change, positive yearly change indicates degradation.

\begin{tabular}{|c|c|c|c|c|c|c|c|}
\hline Site & $\begin{array}{r}\text { Tag } \\
\text { Number }\end{array}$ & $\begin{array}{l}\text { Mineral } \\
\text { Soil (\%) }\end{array}$ & $\begin{array}{r}\text { Fire } \\
\text { Rings }\end{array}$ & $\begin{array}{r}\text { Tree } \\
\text { Stumps }\end{array}$ & $\begin{array}{r}\text { Tree } \\
\text { Damage }\end{array}$ & $\begin{array}{r}\text { Root } \\
\text { Exposure }\end{array}$ & $\begin{array}{r}\text { Latitude }\left({ }^{\circ} \mathrm{N}\right) \\
\text { Longitude }\left({ }^{\circ} \mathrm{W}\right)\end{array}$ \\
\hline \multirow[t]{4}{*}{$\begin{array}{l}\text { Quicksand } \\
\text { North }\end{array}$} & 27 & $\begin{array}{r}66.75 \\
(6.25)^{\mathrm{a}}\end{array}$ & $\begin{array}{r}0.00 \\
(0.00)^{\mathrm{a}}\end{array}$ & $\begin{array}{r}0.00 \\
(0.00)^{\mathrm{a}}\end{array}$ & $\begin{array}{r}1.00 \\
(0.00)^{\mathrm{a}}\end{array}$ & $\begin{array}{r}1.00 \\
(0.00)^{\mathrm{a}}\end{array}$ & $\begin{array}{r}59.7886 \\
149.7885\end{array}$ \\
\hline & 28 & $\begin{array}{r}66.75 \\
(6.25)^{\mathrm{a}}\end{array}$ & $\begin{array}{r}0.00 \\
(0.00)^{\mathrm{a}}\end{array}$ & $\begin{array}{r}0.00 \\
(0.00)^{\mathrm{a}}\end{array}$ & $\begin{array}{r}1.00 \\
(0.00)^{\mathrm{a}}\end{array}$ & $\begin{array}{r}1.00 \\
(0.00)^{\mathrm{a}}\end{array}$ & $\begin{array}{r}59.7888 \\
149.7884\end{array}$ \\
\hline & 30 & $\begin{array}{r}35.00 \\
(-6.50)^{\mathrm{a}}\end{array}$ & $\begin{array}{r}0.00 \\
(0.00)^{\mathrm{a}}\end{array}$ & $\begin{array}{r}0.00 \\
(0.00)^{\mathrm{a}}\end{array}$ & $\begin{array}{r}1.00 \\
(0.00)^{\mathrm{a}}\end{array}$ & $\begin{array}{r}1.00 \\
(0.00)^{\mathrm{a}}\end{array}$ & $\begin{array}{r}59.7880 \\
149.7894\end{array}$ \\
\hline & 83 & $\begin{array}{r}98.00 \\
(0.00)^{\mathrm{a}}\end{array}$ & $\begin{array}{r}0.00 \\
(0.00)^{\mathrm{a}}\end{array}$ & $\begin{array}{r}0.00 \\
(0.00)^{\mathrm{a}}\end{array}$ & $\begin{array}{r}1.00 \\
(0.00)^{\mathrm{a}}\end{array}$ & $\begin{array}{r}1.00 \\
(0.00)^{\mathrm{a}}\end{array}$ & $\begin{array}{r}59.7884 \\
149.7889\end{array}$ \\
\hline \multirow[t]{7}{*}{$\begin{array}{l}\text { Quicksand } \\
\text { South }\end{array}$} & $19^{b}$ & NA & $\begin{array}{r}0.00 \\
(0.00)^{\mathrm{a}}\end{array}$ & $\begin{array}{r}0.00 \\
(0.00)^{\mathrm{a}}\end{array}$ & $\begin{array}{r}1.00 \\
(0.00)^{\mathrm{a}}\end{array}$ & $\begin{array}{r}1.00 \\
(0.00)^{\mathrm{a}}\end{array}$ & $\begin{array}{r}59.7841 \\
149.7897\end{array}$ \\
\hline & $21^{b}$ & NA & $\begin{array}{r}0.00 \\
(0.00)^{\mathrm{a}}\end{array}$ & $\begin{array}{r}0.00 \\
(0.00)^{\mathrm{a}}\end{array}$ & $\begin{array}{r}-0.50 \\
(0.50)^{\mathrm{a}}\end{array}$ & $\begin{array}{r}1.00 \\
(0.00)^{\mathrm{a}}\end{array}$ & $\begin{array}{r}59.7840 \\
149.7896\end{array}$ \\
\hline & 22 & NA & $\begin{array}{r}0.00 \\
(0.00)^{\mathrm{a}}\end{array}$ & $\begin{array}{r}0.00 \\
(0.00)^{\mathrm{a}}\end{array}$ & $\begin{array}{r}1.00 \\
(0.00)^{\mathrm{a}}\end{array}$ & $\begin{array}{r}1.00 \\
(0.00)^{\mathrm{a}}\end{array}$ & $\begin{array}{r}59.7835 \\
149.7895\end{array}$ \\
\hline & $23^{b}$ & NA & $\begin{array}{r}0.00 \\
(0.00)^{\mathrm{a}}\end{array}$ & $\begin{array}{r}0.00 \\
(0.00)^{\mathrm{a}}\end{array}$ & $\begin{array}{r}1.00 \\
(0.00)^{\mathrm{a}}\end{array}$ & $\begin{array}{r}1.00 \\
(0.00)^{\mathrm{a}}\end{array}$ & $\begin{array}{r}59.7831 \\
149.7891\end{array}$ \\
\hline & 24 & $\begin{array}{r}71.75 \\
(-11.25)^{\mathrm{a}}\end{array}$ & $\begin{array}{r}0.00 \\
(0.00)^{\mathrm{a}}\end{array}$ & $\begin{array}{r}0.00 \\
(0.00)^{\mathrm{a}}\end{array}$ & $\begin{array}{r}1.00 \\
(0.00)^{\mathrm{a}}\end{array}$ & $\begin{array}{r}-0.50 \\
(0.50)^{\mathrm{a}}\end{array}$ & $\begin{array}{r}59.7827 \\
149.7889\end{array}$ \\
\hline & 25 & $\begin{array}{r}66.75 \\
(6.25)^{\mathrm{a}}\end{array}$ & $\begin{array}{r}0.00 \\
(0.00)^{\mathrm{a}}\end{array}$ & $\begin{array}{r}0.00 \\
(0.00)^{\mathrm{a}}\end{array}$ & $\begin{array}{r}1.00 \\
(0.00)^{\mathrm{a}}\end{array}$ & $\begin{array}{r}1.00 \\
(0.00)^{\mathrm{a}}\end{array}$ & $\begin{array}{r}59.7826 \\
149.7889\end{array}$ \\
\hline & 26 & $\begin{array}{r}63.00 \\
(0.00)^{\mathrm{a}}\end{array}$ & $\begin{array}{r}0.00 \\
(0.00)^{\mathrm{a}}\end{array}$ & $\begin{array}{r}0.00 \\
(0.00)^{\mathrm{a}}\end{array}$ & $\begin{array}{r}1.00 \\
(0.00)^{\mathrm{a}}\end{array}$ & $\begin{array}{r}1.00 \\
(0.00)^{\mathrm{a}}\end{array}$ & $\begin{array}{r}59.7828 \\
149.7891\end{array}$ \\
\hline \multirow[t]{4}{*}{ Southwestern } & 56 & $\begin{array}{l}98.00 \\
(0.00)\end{array}$ & $\begin{array}{r}0.00 \\
(0.00)^{\mathrm{a}}\end{array}$ & $\begin{array}{r}0.00 \\
(0.00)^{\mathrm{a}}\end{array}$ & $\begin{array}{r}2.71 \\
(-0.29)^{\mathrm{a}}\end{array}$ & $\begin{array}{r}2.71 \\
(-0.29)^{\mathrm{a}}\end{array}$ & $\begin{array}{r}59.7641 \\
150.0605\end{array}$ \\
\hline & 57 & $\begin{array}{r}98.00 \\
(0.00)^{\mathrm{a}}\end{array}$ & $\begin{array}{r}0.00 \\
(0.00)^{\mathrm{a}}\end{array}$ & $\begin{array}{r}0.00 \\
(0.00)^{\mathrm{a}}\end{array}$ & $\begin{array}{r}1.00 \\
(0.00)^{\mathrm{a}}\end{array}$ & $\begin{array}{r}1.00 \\
(0.00)^{\mathrm{a}}\end{array}$ & $\begin{array}{r}59.7643 \\
150.0605\end{array}$ \\
\hline & 61 & $\begin{array}{r}98.00 \\
(0.00)^{\mathrm{a}}\end{array}$ & $\begin{array}{r}0.00 \\
(0.00)^{\mathrm{a}}\end{array}$ & $\begin{array}{r}0.00 \\
(0.00)^{\mathrm{a}}\end{array}$ & $\begin{array}{r}1.00 \\
(0.00)^{\mathrm{a}}\end{array}$ & $\begin{array}{r}1.00 \\
(0.00)^{\mathrm{a}}\end{array}$ & $\begin{array}{r}59.7640 \\
150.0608\end{array}$ \\
\hline & 94 & $\begin{array}{r}98.00 \\
(0.00)^{\mathrm{a}}\end{array}$ & $\begin{array}{r}0.00 \\
(0.00)^{\mathrm{a}}\end{array}$ & $\begin{array}{r}0.00 \\
(0.00)^{\mathrm{a}}\end{array}$ & $\begin{array}{r}3.50 \\
(-0.50)^{\mathrm{a}}\end{array}$ & $\begin{array}{r}3.50 \\
(-0.50)^{a}\end{array}$ & $\begin{array}{r}59.7644 \\
150.0602\end{array}$ \\
\hline
\end{tabular}

a Sample size of 2. P-value is not attainable.

${ }^{b}$ Recovered campsite

${ }^{c} \mathrm{P}$-value $<0.05$ 
Table A1b (continued). Indicator variable baseline (intercept) and annual change (slope) estimates by campsite. Variable baseline of predicted 2008 value (Yearly Change). For all variables, a negative yearly change indicates improvement, 0 is no change, positive yearly change indicates degradation.

\begin{tabular}{|c|c|c|c|c|c|c|c|}
\hline Site & $\begin{array}{r}\text { Tag } \\
\text { Number }\end{array}$ & $\begin{array}{l}\text { Mineral } \\
\text { Soil (\%) }\end{array}$ & $\begin{array}{r}\text { Fire } \\
\text { Rings }\end{array}$ & $\begin{array}{r}\text { Tree } \\
\text { Stumps }\end{array}$ & $\begin{array}{r}\text { Tree } \\
\text { Damage }\end{array}$ & $\begin{array}{r}\text { Root } \\
\text { Exposure }\end{array}$ & $\begin{array}{r}\text { Latitude }\left({ }^{\circ} \mathrm{N}\right) \\
\text { Longitude }\left({ }^{\circ} \mathrm{W}\right)\end{array}$ \\
\hline \multirow[t]{2}{*}{$\begin{array}{l}\text { Southwestern } \\
\text { (continued) }\end{array}$} & 95 & $\begin{array}{r}85.50 \\
(0.00)^{\mathrm{a}}\end{array}$ & $\begin{array}{r}0.00 \\
(0.00)^{\mathrm{a}}\end{array}$ & $\begin{array}{r}0.00 \\
(0.00)^{a}\end{array}$ & $\begin{array}{r}3.50 \\
(-0.50)^{\mathrm{a}}\end{array}$ & $\begin{array}{r}3.50 \\
(-0.50)^{\mathrm{a}}\end{array}$ & $\begin{array}{r}59.7644 \\
150.0604\end{array}$ \\
\hline & $107^{b}$ & NA & NA & NA & NA & NA & $\begin{array}{r}59.7643 \\
150.0606\end{array}$ \\
\hline Sunlight & 66 & $\begin{array}{r}83.00 \\
(-22.50)^{\mathrm{a}}\end{array}$ & $\begin{array}{r}0.00 \\
(0.00)^{\mathrm{a}}\end{array}$ & $\begin{array}{r}0.00 \\
(0.00)^{\mathrm{a}}\end{array}$ & $\begin{array}{r}1.00 \\
(1.00)^{\mathrm{a}}\end{array}$ & $\begin{array}{r}1.00 \\
(1.00)^{\mathrm{a}}\end{array}$ & $\begin{array}{r}59.7493 \\
149.9845\end{array}$ \\
\hline \multirow[t]{7}{*}{ Verdant } & 63 & $\begin{array}{l}98.00 \\
(0.00)\end{array}$ & $\begin{array}{r}1.29 \\
(-0.29)\end{array}$ & $\begin{array}{r}0.00 \\
(0.00)^{\mathrm{a}}\end{array}$ & $\begin{array}{r}1.00 \\
(0.00)^{\mathrm{a}}\end{array}$ & $\begin{array}{r}1.00 \\
(0.00)^{\mathrm{a}}\end{array}$ & $\begin{array}{r}59.6986 \\
149.7329\end{array}$ \\
\hline & 70 & $\begin{array}{l}74.79 \\
(4.46)\end{array}$ & $\begin{array}{r}0.29 \\
(0.21)\end{array}$ & $\begin{array}{r}3.86 \\
(-0.86)\end{array}$ & $\begin{array}{r}0.14 \\
(0.36)^{\mathrm{a}}\end{array}$ & $\begin{array}{r}1.00 \\
(0.00)^{\mathrm{a}}\end{array}$ & $\begin{array}{r}59.6968 \\
149.7387\end{array}$ \\
\hline & 71 & $\begin{array}{l}74.79 \\
(4.46)\end{array}$ & $\begin{array}{r}1.29 \\
(-0.29)\end{array}$ & $\begin{array}{r}-2.57 \\
(1.07)\end{array}$ & $\begin{array}{r}1.00 \\
(0.00)^{\mathrm{a}}\end{array}$ & $\begin{array}{r}2.86 \\
(-0.36)^{a}\end{array}$ & $\begin{array}{r}59.6967 \\
149.7388\end{array}$ \\
\hline & 86 & $\begin{array}{l}98.00 \\
(0.00)\end{array}$ & $\begin{array}{r}-0.86 \\
(0.36)\end{array}$ & $\begin{array}{r}0.00 \\
(0.00)^{\mathrm{a}}\end{array}$ & $\begin{array}{r}1.00 \\
(0.00)^{\mathrm{a}}\end{array}$ & $\begin{array}{r}1.00 \\
(0.00)^{\mathrm{a}}\end{array}$ & $\begin{array}{r}59.6986 \\
149.7331\end{array}$ \\
\hline & $87^{b}$ & NA & $\begin{array}{r}0.00 \\
(0.00)^{\mathrm{a}}\end{array}$ & $\begin{array}{r}0.00 \\
(0.00)^{\mathrm{a}}\end{array}$ & $\begin{array}{r}1.00 \\
(0.00)^{\mathrm{a}}\end{array}$ & $\begin{array}{r}1.00 \\
(0.00)^{\mathrm{a}}\end{array}$ & $\begin{array}{r}59.6982 \\
149.7340\end{array}$ \\
\hline & $105^{b}$ & NA & $\begin{array}{r}0.00 \\
(0.00)^{\mathrm{a}}\end{array}$ & $\begin{array}{r}0.00 \\
(0.00)^{\mathrm{a}}\end{array}$ & $\begin{array}{r}1.00 \\
(0.00)^{\mathrm{a}}\end{array}$ & $\begin{array}{r}1.00 \\
(0.00)^{\mathrm{a}}\end{array}$ & $\begin{array}{r}59.6984 \\
149.7334\end{array}$ \\
\hline & 106 & $\begin{array}{r}150.50 \\
(-17.50)^{\mathrm{a}}\end{array}$ & $\begin{array}{r}0.00 \\
(0.00)^{\mathrm{a}}\end{array}$ & $\begin{array}{r}0.00 \\
(0.00)^{\mathrm{a}}\end{array}$ & $\begin{array}{r}1.00 \\
(0.00)^{\mathrm{a}}\end{array}$ & $\begin{array}{r}1.00 \\
(0.00)^{\mathrm{a}}\end{array}$ & $\begin{array}{r}59.6981 \\
149.7343\end{array}$ \\
\hline
\end{tabular}

a Sample size of 2. P-value is not attainable.

${ }^{\mathrm{b}}$ Recovered campsite

c P-value $<0.05$ 
Table A2a. Indicator variable baseline (intercept) and annual change (slope) estimates by park region. Variable baseline of predicted 2008 value (Yearly Change). For all variables, a negative yearly change indicates improvement, 0 is no change, positive yearly change indicates degradation.

\begin{tabular}{lrrrrrrrr}
\hline Region & Area $\left(\mathbf{m}^{2}\right)$ & $\begin{array}{r}\text { Condition } \\
\text { Class }\end{array}$ & $\begin{array}{r}\text { Vegetation } \\
\text { Loss (\%) }\end{array}$ & $\begin{array}{r}\text { Tent } \\
\text { Rocks }\end{array}$ & Trails & $\begin{array}{r}\text { Ghost } \\
\text { Tree } \\
\text { Damage }\end{array}$ & Trash & $\begin{array}{r}\text { Human } \\
\text { Waste }\end{array}$ \\
\hline Aialik Bay & 66.79 & 3.64 & -42.31 & 10.55 & 2.64 & 0.72 & 0.44 & 0.02 \\
& $(-11.05)^{\mathrm{a}}$ & $(-0.44)^{\mathrm{a}}$ & $(1.42)$ & $(-0.85)^{\mathrm{b}}$ & $(-0.14)^{\mathrm{b}}$ & $(-0.09)^{\mathrm{b}}$ & $(0.13)^{\mathrm{a}}$ & $(0.00)$ \\
Northwestern & 66.99 & 2.43 & -74.96 & 11.47 & 1.40 & 0.00 & 0.00 & 0.00 \\
Fjord & $(-7.61)^{\mathrm{b}}$ & $(-0.06)$ & $(6.70)$ & $(-0.37)$ & $(0.23)^{\mathrm{b}}$ & $(0.00)$ & $(0.00)$ & $(0.00)$ \\
Resurrection & 78.36 & 3.41 & -56.19 & 16.80 & 2.00 & 0.18 & 0.34 & 0.00 \\
Bay & $(-12.78)^{\mathrm{b}}$ & $(-0.48)^{\mathrm{b}}$ & $(-4.30)$ & $(-2.04)$ & $(-0.07)$ & $(-0.03)$ & $(0.27)^{\mathrm{b}}$ & $(0.00)$ \\
\hline
\end{tabular}

\footnotetext{
a P-value $<0.05$

b P-value $<0.001$
}

Table A2b. Indicator variable baseline (intercept) and annual change (slope) estimates by park region. Variable baseline of predicted 2008 value (Yearly Change). For all variables, a negative yearly change indicates improvement, 0 is no change, positive yearly change indicates degradation.

\begin{tabular}{lrrrrr}
\hline Region & $\begin{array}{r}\text { Mineral } \\
\text { Soil (\%) }\end{array}$ & $\begin{array}{r}\text { Fire } \\
\text { Rings }\end{array}$ & $\begin{array}{r}\text { Tree } \\
\text { Stumps }\end{array}$ & $\begin{array}{r}\text { Tree } \\
\text { Damage }\end{array}$ & $\begin{array}{r}\text { Root } \\
\text { Exposure }\end{array}$ \\
\hline Aialik Bay & 62.35 & 0.14 & 0.13 & 0.81 & 0.82 \\
& $(-0.28)$ & $(0.01)$ & $(0.03)$ & $(0.00)$ & $(0.03)$ \\
Northwestern Fjord & 63.90 & 0.13 & 0.34 & 1.66 & 1.54 \\
& $(-2.17)$ & $(-0.01)$ & $(-0.07)$ & $(-0.23)^{\mathrm{b}}$ & $(-0.20)^{\mathrm{b}}$ \\
Resurrection Bay & 74.59 & 0.46 & 0.00 & 1.57 & 1.44 \\
& $(-5.05)$ & $(-0.04)$ & $(0.00)$ & $(-0.20)$ & $(-0.23)^{\mathrm{a}}$ \\
\hline
\end{tabular}

\footnotetext{
a P-value $<0.05$

b P-value $<0.001$
} 
The Department of the Interior protects and manages the nation's natural resources and cultural heritage; provides scientific and other information about those resources; and honors its special responsibilities to American Indians, Alaska Natives, and affiliated Island Communities.

NPS 186/176999, August 2021 
Thional Park Service

U.S. Department of the Interior

Natural Resource Stewardship and Science

1201 Oakridge Drive, Suite 150

Fort Collins, CO 80525 